\title{
ABSTRACTS COLLECTION ACNP 58th Annual Meeting: Author Index
}

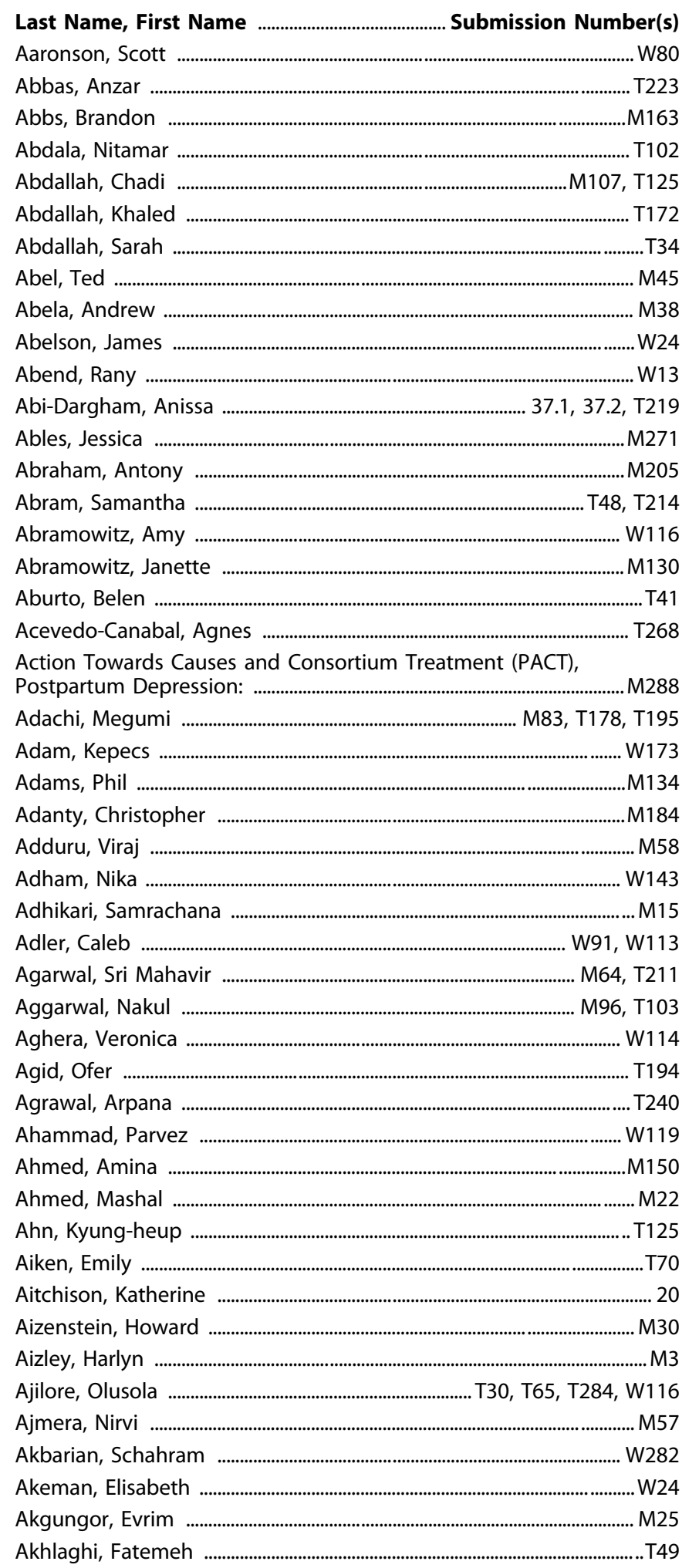

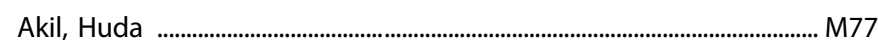

Akli, Said ....................................................................................................... T112

Akre, Olaf ......................................................................................................W21

Alan, Davis ...................................................................................................... W102

Albott, Cristina ...................................................................................................... M142

Alcorn III, Joseph .......................................................................................... T249

Alcover, Karl .................................................................................................. W273

Alexander, Andrew ......................................................................................... T103

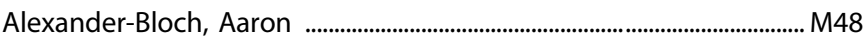

Alexopoulos, George ...................................................................... M130, W178

Aleyasin, Hossein ......................................................................................... M13

Alganem, Khaled ..........................................................................W147, W177

Al-Hasani, Ream .................................................................................. M65, M234

Alia-Klein, Nelly .............................................................................T204, W277

Alim, Tanya ........................................................................................M277, W64

Alkam, Tursun ............................................................................................. W146

Alkhars, Hussain M. .............................................................................................T99

Allen, John .............................................................................................................M201

Allerup, Peter ................................................................................................... W176

Almasy, Laura ................................................................................. 213, T131

Almeida, Jorge .............................................................................................. T130

Almér, Lisa ................................................................................................... T196

Almorsy, Ammar ........................................................................................... T90

Alquisola, Austin ........................................................................................W146

Alrohaibani, Alaaeddin ................................................................................ T198

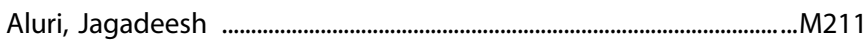

Alvarez, Jean-Claude .................................................................................. T137

Alvarez, Veronica ............................................................................. 17.1, M254

Amaral, David .............................................................................................. W185

Ameis, Stephanie ……………………………………………...........T92, W35

Ament, Seth ............................................................................................... T131

Ameriks, Michael ............................................................................................. W152

Amiri, Anahita ....................................................................................................... T9

An, Xianli ....................................................................................................................... W29

Anacker, Christoph ........................................................................................... M104

Anagnostou, Evdokia ................................................................................. T92

Ananth, Mala ................................................................................................W4, W97

Anastasio, Noelle ...............................................................................M238, T249

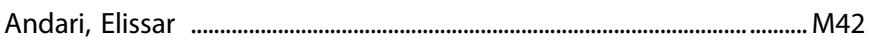

Andersen, Susan ..........................................................................................W278

Anderson, Brian ......................................................................................W120

Anderson, David ........................................................................................ T226

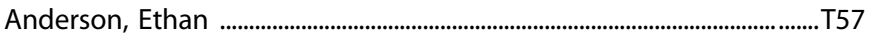

Anderson, Karen .................................................................................................M146

Anderson, Nathan .......................................................................................T66

Andorn, Anne ...............................................................................................161

Andra, Syam ................................................................................................... W5

Andrade, Allyson ..................................................................................................M227

Andrade, Nadja ............................................................................................................W7 
Andrea Jackowski, Andrea .......................................................................138

Andreescu, Carmen .......................................................................................... M30

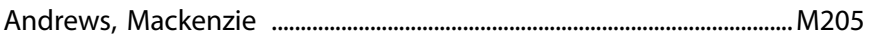

Andrjevic, David ............................................................................................... M34

Andryskova, Lenka ...................................................................................... M74

Angarita, Gustavo

M270, T25

Angarita-Africano, Gustavo

M241, W10, W219

Angstadt, Mike

W240

Ansorge, Mark

Anthony, Jim

T97

M268, W273

Anticevic, Alan .M168

Anton, Raymond

M251, T159, W272

Antonson, Adrienne ...............................................................................................W37

Ao, Mei .............................................................................................. W129

Applebey, Sarah ................................................................................................M232

Apud, Jose ................................................................................................... W211

Arackal, Joel .....................................................................................................129

Arango, Celso ……………………………………………………..... W42, W176

Arango, Victoria ................................................................................. 30.1, W131

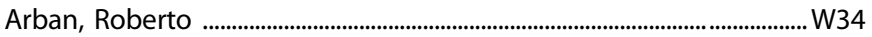

Arcos-Burgos, Mauricio ............................................................................... W128

Ardekani, Cory .............................................................................................. T234

Arey, Rachel ....................................................................................................T67

Argemi, Josepmaria ...................................................................................... W229

Argyelan, Miklos ............................................................................M168, W90

Arias, Albert ..................................................................................T249, W136

Arkin, Sophie .............................................................................................. W199

Armey, Michael .......................................................................................................28

Armstrong, Michael ......................................................................................... T181

Armstrong, William ..........................................................................................M186

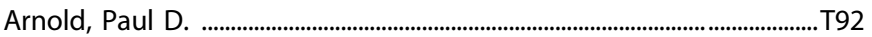

Aronson Fischell, Sarah .............................................................................. W258

Arora, Manish ...........................................................................................W57

Arout, Caroline .................................................................................................. T33

Arriaga, Moises ................................................................................................M250

Arscott, Kelly .................................................................................................. W142

Arver, Stefan ..................................................................................................... M71

Arzberger, Thomas .................................................................................. T224

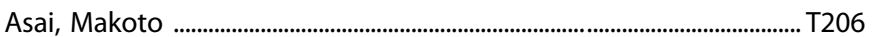

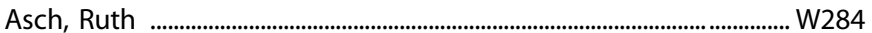

Aselcioglu, Irem ............................................................................................. W283

Asgharnejad, Mahnaz .....................................................................................W74

Ashamalla, Sandra ........................................................................................ T140

Ashirova, Elina ....................................................................................................... M256

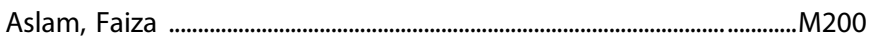

Aslam, Haris ........................................................................ M78, T233, T283

Aslanoglou, Despoina .......................................................................................162

Aspesi, Dario .................................................................................................. W2276

Assaf, Michal ................................................................................................ 224

Assali, Ahlem ................................................................................................. M55

Aston-Jones, Gary .......................................................................................T59

Atkins, Alexandra ............................................................................................. T207

Atlas, Lauren ..........................................................................................................32.1

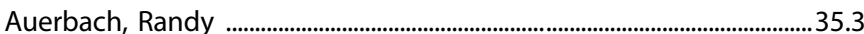

Augier, Eric .............................................................................................................. W251

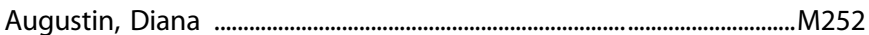

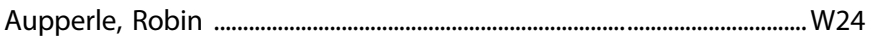

Austin, Christine ...................................................................................................W57

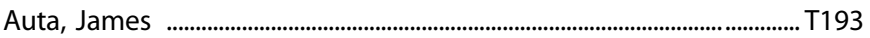

Averill, Christopher .......................................................................................M107

Averill, Lynnette ................................................................................. M107, T125

Avramopoulos, Dimitri ..................................................................................... 18.3

Azevedo, Estefania ................................................................................................T14

Baandrup, Lone ...………………………………………………………... W176

Babasiz, Mari ....................................................................................................W16

Babbs, Richard ....................................................................................M272

Bachi, Keren ......................................................................................... 25.1, 25.4

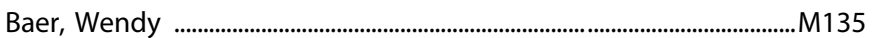

Baganz, Nicole ...................................................................................................... T18

Bagdon, Gabriela ................................................................................................W22

Bagnell, Anna ............................................................................................ T111

Bagot, Rosemary ...................................................................24.1, 24.8, M228

Bahji, Anees .................................................................................................W14

Bai, Xinglong ............................................................................................... T22

Bailey, Michael ...………………………………………………………………...W37

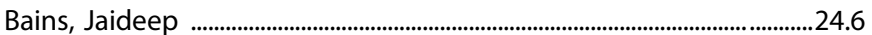

Bajaj, Mira .....................................................................................................W13

Bajor, Laura ........................................................................................................ T149

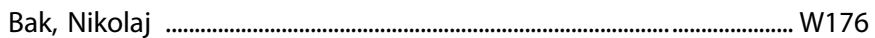

Baker, Dewleen ......................................................................................M215

Baker, Fiona ................................................................................................ M41

Baker, James ........................................................................................................ T130

Bakker, Geor ........................................................................................ M181, M193

Balana, Bartosz .............................................................................................. W117

Baldassarri, Stephen .................................................................................... W219

Balderston, Nicholas ..................................................T3, T241, W22, W283

Baldwin, Elena ............................................................................................. W57

Bale, Tracy ............................................................................. 13, 13.1, T11, T96

Bales, Karen ............................................................................................. T243

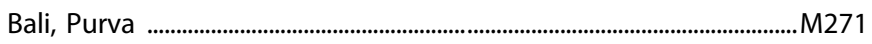

Bali, Vedrana ......................................................................................... W224

Ball, Tali ................................................................................................M16, T71

Ballard, Elizabeth ............................................................................................ 12.4

Ballard, Shannon ......................................................................................... W234

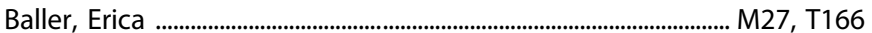

Baller, Joshua ...................................................................................................... T78

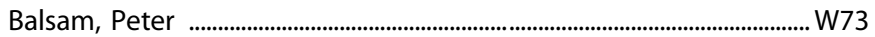

Balu, Darrick .......................................................................................................... W193

Bangasser, Debra ........................................................................................... T2 234

Bani Fatemi, Ali .............................................................................................. T2

Banihashemi, Layla ....................................................................................... T99

Banker, Sarah ........................................................................................... T107

Bankole, Kemi .............................................................................................. T138

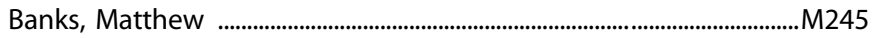

Bannister, Thomas D. ................................................................................. T268

Bannon, Michael .................................................................................................. W271

Baram, Tallie Z. ..................................................................................................T22

Baratta, Annalisa ........................................................................................... W213

Barbeau, Annie ................................................................................................... T172

Barbier, Estelle ..................................................................W229, W238, W251

Barbour, Tracy ..........................................................................................M131, T175 
Barchiesi, Riccardo ........................................................................................... W2 W

Bar-Haim, Yair ....................................................................................................W13

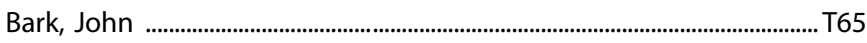

Barker, Jacqueline ....................................................................................... T235

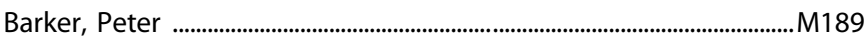

Barko, Kelly ................................................................................................... W241

Barlett, Elizabeth ........................................................................................ W115

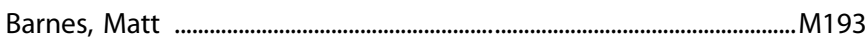

Barnhofer, Thorsten ............................................................................................. W84

Barr, Dana Boyd ............................................................................................ T198

Barr, Jamie

T264

Barredo, Jennifer

M158, T64

Barrett, Frederick 59.4, M226, W102

Barrientos, Ruth ............................................................................................... T66

Barrow, James ................................................................................................ M285

Bartel, Laura .................................................................................................. W199

Barthelemy, Christine .......................................................................287, W59

Bartlett, Elizabeth ...........................................................................................W97

Bartley, Christopher ..................................................................................... W207

Barton, Bruce ....................................................................................................... M130

Baruah, Jugajyoti ...................................................................................... W193

Barzilay, Ran ........................................................................................... M27, T93

Bass, Caroline ................................................................................................ M257

Bassareo, Valentina ....................................................................................... T245

Basset, Danielle ............................................................................................... M48

Bassir Nia, Anahita ................................................................................................... W31

Bastias, Cristian ............................................................................................M273

Bataller, Ramon .............................................................................................. W229

Batchelor, Hannah ......................................................................................... T43

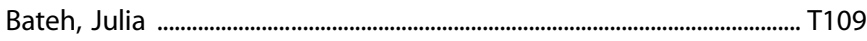

Bathgate, Ross ............................................................................................ T52

Batra, Kanha .............................................................................................. W144

Batuure, Anita ......................................................................................................W21

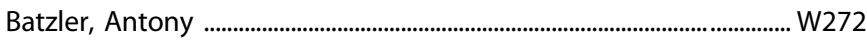

Bauman, Melissa .......................................................................................... W185

Baumann, Michael ...................................................................................... W230

Baune, Bernhard ..........................................................................................W118

Baxi, Madhura ..................................................................................................... W167

Bazer, Allyson ..................................................................................................... T140

Bazmi, Holly .......................................................................................... M182, W184

Beale, Alexis ............................................................................................................W17

Beard, Katie ...................................................................................................... T105

Bearden, Carrie .................................................................................................. T12

Beaulieu, Jean Martin .................................................................................. W209

Bebko, Genna ............................................................................. M78, T233, T283

Beck, Jaclyn ............................................................................................................ T22

Beck, Quincy .................................................................................................. T167

Becker, Howard ............................................................................. T61, W247

Becker-Krail, Darius ...................................................................................... M18

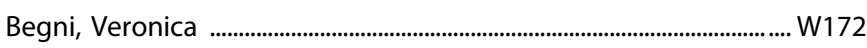

Behrens, Brigid .............................................................................................. T84

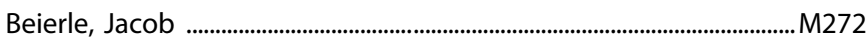

Beierlein, Michael ...............................................................................................W9

Bekhbat, Mandakh ....................................................................................... W101

Bell, Richard ................................................................................................................ T246

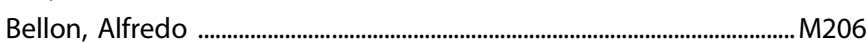

Beltran, Jacqueline .......................................................................................... M278

Benca, Ruth ...........................................................................................................22.2

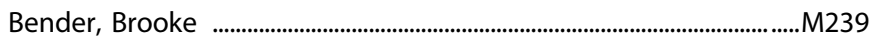

Bendl, Jaroslav .......................................................................................... T22

Benedetti, Francesco ....................................................................................... 12.3

Benkelfat, Chawki ...................................................................................... T238

Benn, Emma ............................................................................................... T117

Bennett, Jeffrey .............................................................................................. W185

Benson, Brenda ................................................................................................M282

Benster, Lindsay ..............................................................................................159

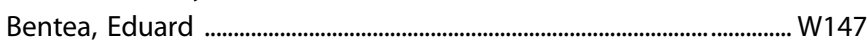

Benton, Mary Lauren ....................................................................................... W52

Benton, Tami ....................................................................................................... T93

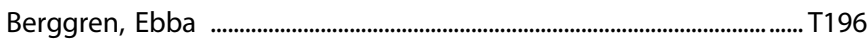

Bergman, Elizabeth M. .............................................................................W12

Bergman, Jack .....................................................................T266, T270, W110

Bergman, Jeffrey ....................................................................................... W103

Berk, Michael ...................................................................................................T28

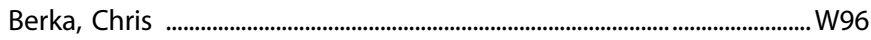

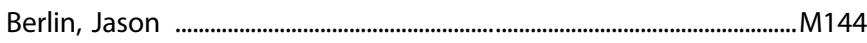

Berlow, Yosef ...................................................................................................115

Berman, Karen ...................................... M29, M283, T166, T199, W40, W211

Berman, Mitchell ............................................................................................ T123

Berman, Rebecca ........................................................................................... W199

Berner, Laura .....................................................................................................................2. 27.3

Bernstein, David ............................................................................................M259

Bernstein, Hans-Gert ......................................................................................... M166

Berrettini, Wade ................................................................................. 15.5, 41, 47

Berroteran Infante, Neydher ................................................................... W127

Bershad, Anya ...................................................................................... M112, M223

Bertera, Suzanne ..........................................................................................162

Bertocci, Michele ..............................................M78, T99, T283, W103, W135

Bertolino, Alessandro .............................................................................. W209

Bertolino, Alina .........................................................................................W165

Bertz, Jeremiah .............................................................................W254, W256

Berwian, Isabel ...........................................................................................W86

Besich, Zach .................................................................................................M199

Bessette, Katie ...................................................................................................... T30

Bettini, Ezio ........................................................................................................... T226

Betzhold, Sara ............................................................................... M4, W70, W255

Beurmann, Silvia ......................................................................................... T272

Bewernick, Bettina ........................................................................................... W124

Beydler, Emily ...................................................................................................W17

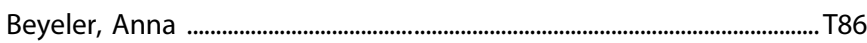

Bhakta, Savita ...................................................................................... M159, T40

Bhargava, Aditi ...........................................................................................W19

Bhatt, Shivani ....................................................................................T47, W10

Bhattacharya, Anindya ............................................................ 48, T139, W96

Bhattacharya, Tushar K. ..........................................................................M247

Bhattacharyoya, Sagnik .............................................................................. M242

Bhatte, Sai ...................................................................................................... T106

Bi, Guo-hua ................................................................................. T50, W271

Bice, Collette .................................................................................................. M63

Bicks, Lucy .................................................................................................... W282

Bidwell, L. Cinnamon ........................................................................W220, W243

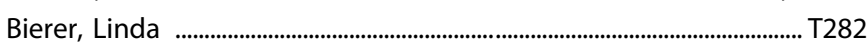


Bierlein-De La Rosa, Gabrielle

.. T258

Biernacka, Joanna

W137, W265, W272

Biesecker, Catherine

..M275

Bigio, Benedetta

T142, W89

Bilbo, Staci

48.2, W36

Bilodeau-Houle, Alexe

M50

Binder, Devin

.. M40

Binder, Elisabeth B.

. T224

Biondo, Francesca

. .26 .3$

Bioque, Miquel

W118

Birmaher, Boris

W103, W135

Birnbaum, Rebecca

M175

Bisby, James

..... 13

Bishop, Jeffrey

..T37, T78

Bishop, Sonia

.52 .5

Bittar, Thibault

. T172

Bitter, Istvan

..M163

Bizon, Jennifer

M4, T2, W70, W72, W255

Bjork, James

... T249

Blackford, Jennifer

49, W166

Blaes, Shelby

W255

Blair, Joshua

M120

Blakely, Randy

T186

Blanchette, Brittney

M158

Blanco, Carlos

Blangero, John

Blank, Brandon

M48, T131

Blanpain, Lou

Blasi, Giuseppe

M176

W209

Blazer, Annie

M172

Blednov, Yuri

. T246

Blennow, Kaj

M173, T158

Bliss, Sarah .

... 108

Bloch, Michael

M60, M136, T34

Bloemen, Oswald

.M181

Blokhin, Ilya W229

Blumberg, Hilary

Blumberger, Daniel

... 35

.M191

W181

Bobadilla, Ana-Clara

....T54

Bocarsly, Miriam

Bodurka, Jerzy

Boehm, Matthew

...M254

Bohlen, Joseph

108, T121, W24

M232, W141

... M51

Bohn, Laura

$.41 .2, \mathrm{~T} 268$

Bohon, Cara

..M127

Boileau, Isabelle

Boivin, Michel

Bojanek, Erin $\mathrm{K}$.

Bolanos, Carlos

Bolbos, Radu

Boldrini, Maura

Bollinger, Justin

Bolshakov, Vadim

Bolton, Jessica

Bolton, Kirsten
Bomyea, Jessica

M16

Bonar, Lisa

T99, W103, W135

Bonaventura, Jordi

M232, W141, W246, W271

Bonaventure, Pascal

W78, W100

Bonci, Antonello

W141, W250

Bonomo, Yvonne

....T52

Bonsall, David R.

M174

Bonthapally, Vijayveer

T127

Booij, Jan

M181

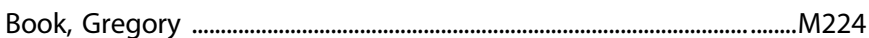

Book, Sarah .............................................................................................................. M251

Boos, Alison .............................................................................................. T2 14

Boost, Carola ...................................................................................................18

Borden, Phillip ............................................................................................... W227

Bordey, Angelique ......................................................................................... T9

Borkar, Chandrashekhar ............................................................................ W28

Borlido, Carol ................................................................................................. T21

Born, Jan .......................................................................................................... T44

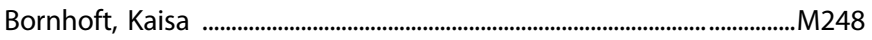

Borrego, Marissa ..........................................................................................M267

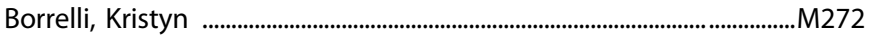

Borron, Ben .............................................................................................. T163

Borroto-Escuela, Dasiel ................................................................................... 9.4

Bosc, Marion ...............................................................................................W173

Bosini Remus, Isadora ..............................................................................185

Bossert, Jennifer ..................................................................................................... T55

Bottino, Rita .........................................................................................M162

Boukezzi, Sarah ................................................................................... M5, T168

Bousman, Chad ..................................................................................... 20.4, T151

Bowden, Charles L. ......................................................................................W98

Bowden, Sylvie ............................................................................................ T21

Bowers, M. Scott ........................................................................................... M247

Boyd, Rhonda ............................................................................................ T93

Bozaoglu, Kiymet ............................................................................................T52

Bradberry, Charles ...........................................................................T273, W141

Bradley, Bekh ..................................................................................................... T8

Brady, Lillian ........................................................................................... T279

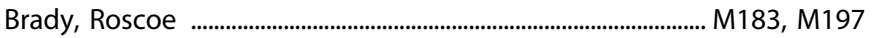

Braff, David ......................................................................................M210, T210

Braga, Raphael ................................................................................................. W90

Braimah, Adebayo ......................................................................................... W28

Brand, Harrison ............................................................................................W52

Brandl, Eva ................................................................................................. W196

Brandt, Simon ..................................................................................................... W230

Branham, Margaret ..................................................................................... W1

Bravo-Rivera, Christian .................................................................................... 15.4

Bravo-Rivera, Hector ......................................................................................... T82

Brazdil, Milan ................................................................................................. M74

Bremer, Paul ................................................................................................. M245

Bremmer, Michael .......................................................................................... $\mathrm{M} 112$

Brenhouse, Heather ..................................................................................... T91

Brennand, Kristen .....................................................................18, 18.2, W52

Brenner, Helena ........................................................................................ T159

Brenner, Robert ............................................................................................... T154

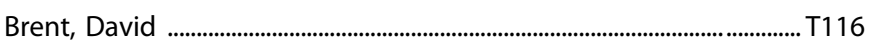

Breslin, Florence ...............................................................................................W41 
Bressan, Rodrigo ............................................................................................138

Bretton, Zachary ..............................................................................................M125

Brian, Kopell

.T184

Briand, Lisa

T234, W259

Brick, Leslie

.M287

Brickner, Megan ......................................................................................... W218

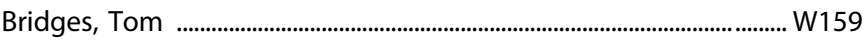

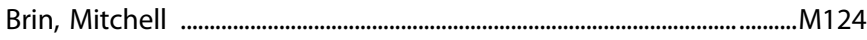

Brinkmalm, Anna ..............................................................................................173

Brislin, Sarah .................................................................................................... W235

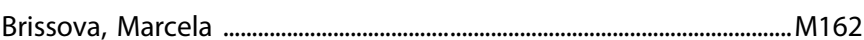

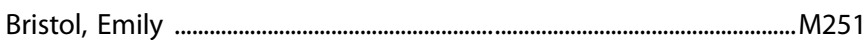

Britton, Jennifer .................................................................................................... M12

Brock, Ashley .........................................................................................................M264

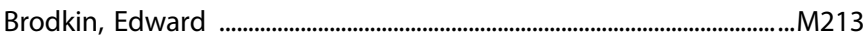

Broos, Hannah .............................................................................................. T242

Brothers, Shaun P. ..........................................................................................W6

Brotman, Melissa .........................................M56, M80, M96, M282, T84, W13

Brown, Alan ..................................................................................................... T42

Brown, Alastair ..................................................................................................193

Brown, Clayton H. ..................................................................................... W180

Brown, E. Sherwood ...................................................................................... M63

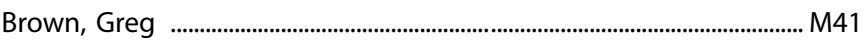

Brown, Imani ......................................................................................................M27

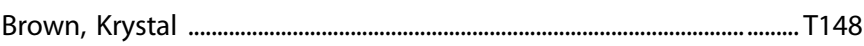

Brown, Lisa .............................................................................................................. T148

Brown, Samantha …………………………………………………………….... T273

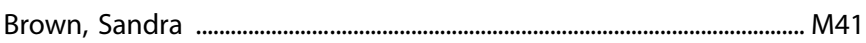

Browne, Caroline .............................................................................................121

Browning, Brittney ............................................................................................. T49

Brownstein, Michael .............................................................................M146, W17

Brucato, Gary ............................................................................................... T219

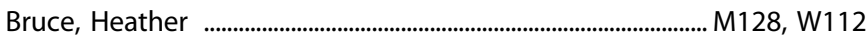

Bruchas, Michael ............................................................................ M260, M265

Bruggeman, Richard .....................................................................................

Brugger, Stefan .............................................................................................

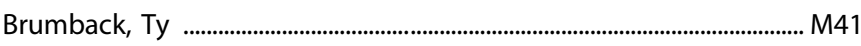

Brunell, David ............................................................................................... T173

Bruno, Davide .................................................................................................... T158

Bryan, Angela .....................................................................................W220, W243

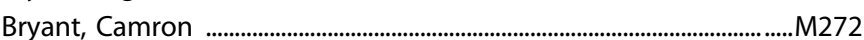

Bu, Xuan ............................................................................................................156

Bublitz, Joshua ............................................................................................... W137

Bubser, Michael ............................................................................................ W159

Bucan, Maja ................................................................................ 22, M213, T131

Bucci, Paola .................................................................................................. W176

Buchanan, Robert ....................................M160, W167, W170, W179, W180

Buchsbaum, Bradley .................................................................................... W183

Buchsbaum, Monte .................................................................................. W183

Buck, Silas .................................................................................................. W213

Buckley, Alex .................................................................................................129

Bugarski-Kirola, Dragana ...........................................................................163

Bugay, Vladislav ...................................................................................... T154

Buhl, Derek .................................................................................................................. W74

Bui, Eric ............................................................................................................................. M15

Bui, Hong ............................................................................................................. M8
Bujarski, Spencer .................................................................................................2253

Bukvić, Nikola ....................................................................................................117

Bumanglag, Argyle ....................................................................................... T2

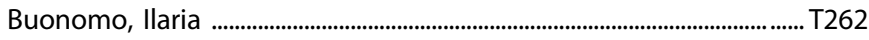

Burdick, Katherine ...................................................... M95, M110, T162, T204

Bureau, Marie ........................................................................................... T226

Burgdorf, Jeffrey ............................................................................... M101, M247

Burgess, Neil ..................................................................................................... T3

Burgess-Hull, Albert .........................................................................W254, W256

Burghardt, Kyle ............................................................................................... T4

Burke, Christine ................................................................................................ T149

Burke, Emily ................................................................................................. M51, M176

Burke, Sara ........................................................................................................ T2

Burket, Jessica ............................................................................................... W44

Burkhouse, Katie ........................................................................................... T30

Burmeister, Jason .......................................................................................M219

Burns, Jamie ......................................................................................................M275

Burwell, Rebecca ........................................................................................... M21

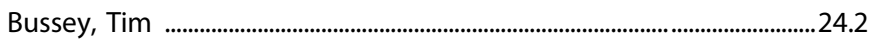

Bustillo, Juan ................................................................................................ T216

Butters, Kim ................................................................................................M120

Buxbaum, Joseph D. ................................................................................... W21

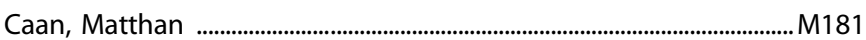

Caballero-Floran, Rene ..................................................................................... T165

Cabello-Arreola, Alejandra ............................................................................ W137

Cabral, Joana .......................................................................................................... W158

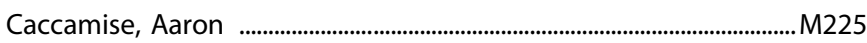

Cacciaglia, Roberto .......................................................................................M276

Caceda, Ricardo ............................................................................................... W115

Caceres, Gabriella ...................................................................................... M42

Cadoni, Cristina ......................................................................................... W249

Cahalan, Michael D. .................................................................................... T22

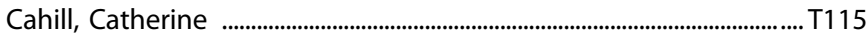

Cai, Denise ......................................................................................... 8, 8.1, T263

Caine, Carolyn ................................................................................................ M49

Calabrese, Joseph .......................................................................................... W123

Caldwell, Sorana ..................................................................................................W32

Calhoun, Amanda ............................................................................................ T117

Calipari, Erin ............................................................................ T89, T132, T279

Calkins, Monica .................................................................27, T93, W200, W202

Callicott, Joseph ................................................................................................M283

Camacho, Mateo ............................................................................................ M44

Camchong, Jazmin ............................................................................... T48, W268

Cameron, Judy ....................................................................................... M33, T106

Campbell, E. Cabrina .............................................................................................. 47.8

Campbell, Morganne ...................................................................................... M51

Campbell-Sills, Laura ................................................................................ M16

Camprodon, Joan ............................................................................. M131, T175

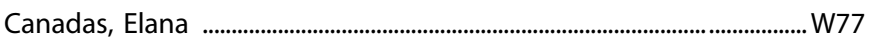

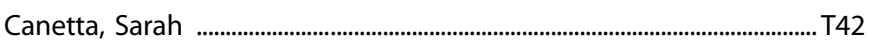

Cannady, Reginald ......................................................................................... T61

Cantos, Adriana ............................................................................................. M84

Cantrell, Elizabeth ...............................................................................................M257

Canuso, Carla .............................................................................................M94, W130

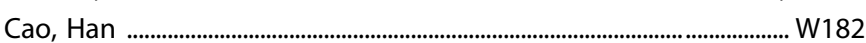
. 
Capra, John ...................................................................................................W52

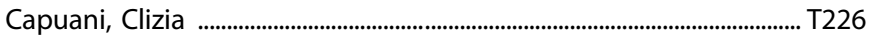

Caputi, Francesca ......................................................................................... W153

Caradonna, Salvatore ...................................................................................... M77

Caravaggio, Fernando .................................................................................. M64

Cardenas, Sofia .............................................................................................. M80

Cardinale, Elise ................................................................................................W13

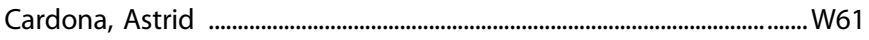

Cardoso, Lauren ..............................................................................................M210

Carelli, Regina ................................................................................................M262

Carlezon, William ............................................................... 19, T10, T80, W1 10

Caro, Keaven ................................................................................T109, W282

Caroff, Stanley ...................................................................................................... T205

Caroline, Browne ...........................................................................................M137

Carpenter, Linda .....................................M32, M158, T64, T74, T167, W104

Carpenter, Rachel ......................................................................................... T234

Carpio, M. J. .................................................................................................M252

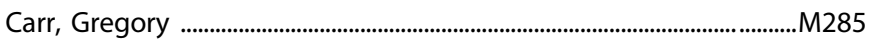

Carrasquillo, Yarimar ........................................................................................ 40.1

Carreno, Flavia .................................................................................................... T154

Carrette, Lieselot ........................................................................................ T264

Carruthers, Nicholas ................................................................................... W152

Carskadon, Mary ..........................................................................................T10

Carson, Richard ..............................................M218, M241, T276, W10, W219

Carter, Cameron ....................................................................... T38, T113, W185

Carter, Sierra ......................................................................................13.3, T8, W23

Casello, Sanne ..............................................................................................M205

Casey, Kathleen ............................................................................................ T130

Casimir, Alexander ..................................................................................... W234

Cassidy, Clifford ..................................................................................................... T1

Castellanos, Francisco ........................................................................ 50.2, T97

Castellanos-Ryan, Natalie ............................................................................ T238

Castillo, Juan .............................................................................................. T252

Castillo-Passi, Rolando ................................................................................... T41

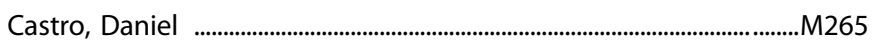

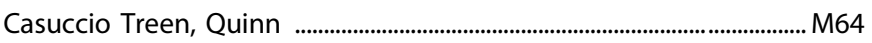

Casuccio-Treen, Quinn ........................................................................... T211

Catalano, Glenn ............................................................................................. T149

Catavero, Christina ......................................................................................... W20

Cates, Hannah ..................................................................................... M228, W231

Cathomas, Flurin ........................................................................ M13, T157, W8

Cattane, Nadia ...............................................................................M92, W172

Cattaneo, Annamaria ....................................................................M92, W172

Cavus, Idil ........................................................................................................ T218

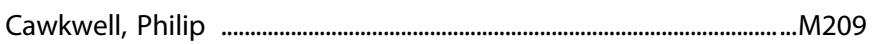

Cayton, Christelle ............................................................................................252

Cearley, Cassia N. ..............................................................................................M247

Cecil, Kim ....................................................................................................... M23

Celebi, Seyda ................................................................................................... T1

Centanni, Samuel ............................................................................................ W248

Cereceo Page, Stephanie ................................................................................. M51

Cerit, Hilal .................................................................................................... T168

Cetin-Karayumak, Suheyla .........................................................W167, W170

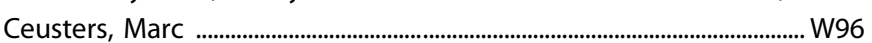

Cha, Jiook ...................................................................................M53, T102, W106

Chadi, Abdallah ................................................................................................... T2
Chaki, Shigeyuki .......................................................................................... T126

Chakrabarty, Paramita ......................................................................................... T2

Chakravarty, Mallar ..................................................................................... W162

Chamberlain, Samuel ....................................................................T122, W154

Chamberlin, Linda .......................................................................................M125

Chamoun, Mira .............................................................................................. T1

Chan, Amy ....................................................................................................... W242

Chan, Chi .................................................................................................. T204

Chan, Kenny ............................................................................................ M13, T157

Chan, Shi Yu ...............................................................................................................M197

Chand, Ganesh ........................................................................................................... M27

Chandra, Joshua .................................................................................................. T2

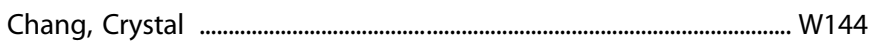

Chang, Lijia ........................................................................................................ W95

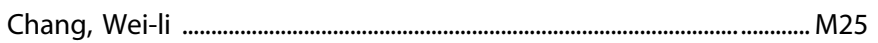

Chanthongdee, Kanat .............................................................................. W238

Charles, Sophia ................................................................................................. T266

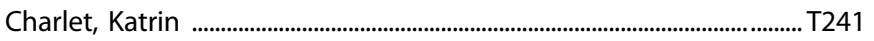

Chase, Henry .......................................................................... M78, T233, T283

Chatzinakos, Christos ..................................................................................... T80

Chatzittofis, Andreas ..................................................................................... M71

Chavarria, Jesus ..............................................................................................

Chavez, Sofia .................................................................................................W162

Chavkin, Charles .................................................................................................M205

Cheer, Joseph ....................................................................... 37.4, T18, T53, T132

Chen, Alan .................................................................................................................... M15

Chen, Alon .................................................................................................................... 24. 24

Chen, Chao ........................................................................................279, W205

Chen, Guang …………………………………………………….......T192, W117

Chen, Haiying .............................................................................................................. T173

Chen, Helen .................................................................................................................W37

Chen, Huei-Ying ............................................................................................. M51

Chen, Jiali ........................................................................................106, T244

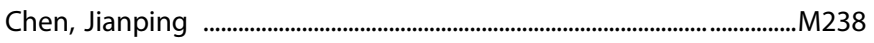

Chen, Kehui ............................................................................................... W184

Chen, Melanie .................................................................................................M272

Chen, Richard .............................................................................................. W123

Chen, Runshu ....................................................................................................... M66

Chen, Shou ..............................................................................................................M160

Chen, $\mathrm{Xi}$................................................................................................................... T22 $\mathrm{T} 2$

Chen, Xiangchuan .................................................................................................... 135

Chen, Ying ............................................................................................................ T271

Chen, $\mathrm{Yu}$.................................................................................................M279, W205

Cheng, Hailong ...................................................................................T215, W204

Cheng, Haoxiang ....................................................................................................... M13

Cheng, Huaiyan .................................................................................................M121

Cherif, Aboubacar ................................................................................................ T16

Chiappelli, Joshua ............................................................M128, M281, W112

Chien, Yi-Ling ............................................................................................... W47

Childress, Anna Rose ............................................................................. W263

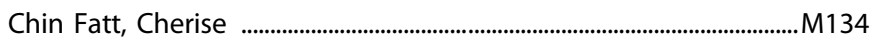

Chin-Fatt, Cherise .................................................................................................... M89

Chintoh, Araba ....................................................................................................... T2 211

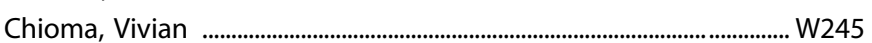

Chirico, Margherita ................................................................................................... M1 19

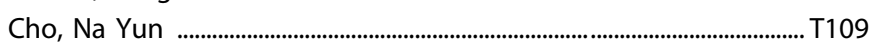


Choi, Doo-Sup

W217

Choi, Ki Sueng

T184

Choi, Yong Kee

...W65

Choo, Tse Hwei

M164

Chou, Tai-Li

Chowdury, Asadur

Christian, Bradley

Christian, Edward

Chukwueke, Chidera C.

M237

Chukwurah, Evelyn

Chuma, Nao

Chung, Daniel

.T111

Chung, Youjin

Churillo, Kristina

W194, W195

... W195

M135

Churilov, Leonid

..T52

Ciesielski, Andrew

M182, W184

Cihakova, Daniela

M160

Cipriani, Nicolo

T204

Cisler, Joshua

$\mathrm{M} 19, \mathrm{~T} 72, \mathrm{~T} 81$

Cisneros, Sebastian

.... M52

Citrome, Leslie

Clancy, Dillon

M68, T136

W214

Clapp, Wesley ............................................................................................ T218

Clark, Ashton ...............................................................................................................T60

Clark, Jeremy .....................................................................................................M240

Clark, Uraina .................................................................................................. T117

Clarke, Emily ...................................................................................................... T85

Clarke, Toni . 1240

Clausen, Ashley

T85, W24

Clayson, Peter .T209

Clayton, Michal

M80, M282

Clementz, Brett

T37, W170

W187

Cleusix, Martine

M159

Clifford, Royce

....M226

Clifton, John

..W87

Clinton, Sarah

Clytus, Janay

Cochran, Amy

T81, T155

Cochran, Ashly

... W89

Codario, Cristina ...u.u.u.u.u.u.u. T106

Cody, Caitlyn

Coenen, Volker M44

Coffey, Christopher

W124, W155

....146

Coffey, Kevin

$\mathrm{M} 133, \mathrm{~T} 265$

Cogan, Elizabeth ................................................................................................ W20

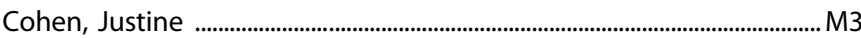

Cohen, Talia ................................................................................................. T221

Cohen-Gilbert, Julia ........................................................................................ M49

Colaizzi, Janna ................................................................................................... W41

Colby, Colin

W137, W272

Cole, Maury

..M229

Cole, Robert

.M235

Colechio, Elizabeth M.

Coleman, Cory

T113

Colibazzi, Tiziano

Colich, Natalie
Collado-Torres, Leonardo

M175

Collazo, Andres

M255

Collier, Jenelle ................................................................................................. M45

Collier, Samuel J. ........................................................................................ T130

Collin, Guusje .................................................................................................. W18

Collins, Anne ...............................................................................................

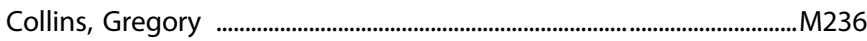

Collins, Paul ........................................................................................................... M57

Collins, Ryan .................................................................................................. W52

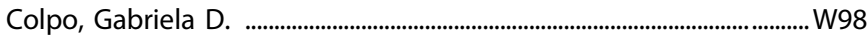

Colucci, Laura ......................................................................................... T211

Compton, Wilson ..................................................................................................30.4

Conelea, Christine ........................................................................................... T105

Congdon, Eliza .......................................................................................... T29

Conn, P. Jeffrey .....................................................................................37.5, T132

Conneely, Karen ............................................................................................... T8

Conroy, Susan .................................................................................................. W122

Consortium, CommonMind ........................................................................ W52

Constable, R. Todd ....................................................................................... W58

Contet, Candice .........................................................................................M255

Contreras, Javier ............................................................................................ T131

Conus, Philippe .............................................................................................. W187

Conway, Charles R. .................................................................................... T148

Conway, Sineadh ..............................................................................................234

Cook, David ....................................................................................................... T185

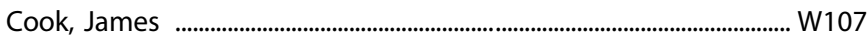

Cook, Philip ............................................................................................................ W283

Coombes, Brandon .........................................................................W265, W272

Coon, William .................................................................................................T63

Cooper, Crystal ................................................................................ M119, M134

Cooper, Jessica .......................................................................................52.3, T170

Cope, Lora .......................................................................... M284, W235, W236

Coppersmith, Daniel .....................................................................................W13

Corder, Gregory .................................................................................................. 6.3

Cornier, Marc-Andre ....................................................................................... W68

Corona, Kitzia ....................................................................................................... M54

Correa, Adriana ................................................................................... T83, W8

Correll, Christoph ............................................................................W203, W204

Cortes, Carlos ............................................................................................ T241

Cortes-Briones, Jose .......................................................................................... T276

Cosgrove, Kelly ........................................................................ M241, T47, W10

Costa-Dookhan, Kenya .................................................................................. T211

Costanzo, Danielle ............................................................................................. W214

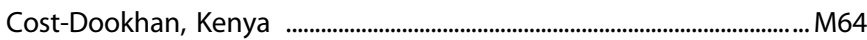

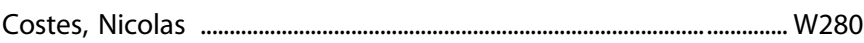

Costigan, Michele ............................................................................................146

Côté, Sylvana ................................................................................................... T238

Coughlin, Jennifer ................................................................................................. 37

Council, Kimaya ....................................................................................................

Covey, Daniel ................................................................................................... T18

Cowan, Christopher ............................................................................... M55, T57

Cowen, Mara .............................................................................................. T111

Cox, Sylvia ML ............................................................................................. T238

Crabbe, John .................................................................................................... T2

Craske, Michelle .....................................................................................................W24

Creed, Meaghan ................................................................................. T115, W75 
Creighton, Samantha ................................................................................ W232

Cressy, Jianna .............................................................................................W15

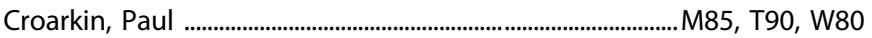

Crosbie, Jennifer ...............................................................................................T92

Cross, Donna ................................................................................................... T185

Crossan, April .................................................................................................W32

Crow, Timothy ................................................................................W167, W170

Crummy, Elizabeth ...................................................................................... W222

Cruz-Martin, Alberto .........................................................................................M272

Csernansky, John ...........................................................................................161

Cuartas, Mauricio .................................................................................................... W128

Cubells, Joseph ............................................................................................. M42

Cudkowicz, Merit ..................................................................................................M146

Cuellar-Barboza, Alfredo ................................................................................ W137

Cuénod, Michel .................................................................................................... W187

Cuesta, Santiago ...........................................................................................T5

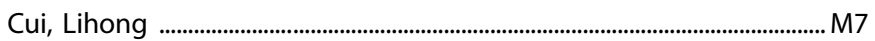

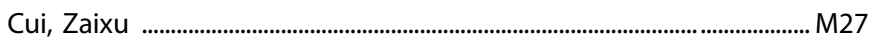

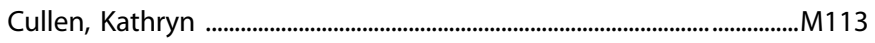

Cullum, C. Munro ………………………………………………………......T156

Cunha, Catarina ............................................................................................... T97

Cunningham, Ashley ....................................................................................M228

Cunningham, Kathryn ...................................................... M238, T249, W253

Curley, James ................................................................................................. W144

Curran, Joanne .................................................................................... M48, T131

Curtin, Paul .................................................................................................................. W5

Curtis, Brenda ......................................................................................................... W25

Curtis, Michael .................................................................................................... T226

Cusimano, Michael ........................................................................................ W62

Cuthbert, Bruce .................................................................................................... T198

Cyr, Marilyn .................................................................................................... T187

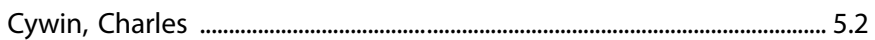

Czaja, Sara .....................................................................................................W2

Czamara, Darina .......................................................................................... T224

Czarapata, Jasmin ...............................................................................M283, W40

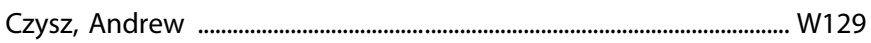

Da Silva, Susana

T225

Da Silva, Tania

$\mathrm{T} 36, \mathrm{~T} 237$

Dabas, Grishma ..................................................................................................... T18

Dada, Oluwagbenga ....................................................................................... T2 17

Dadabayev, Alisher ........................................................................................W33

Dager, Alecia .................................................................................................M224

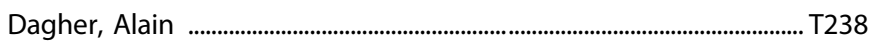

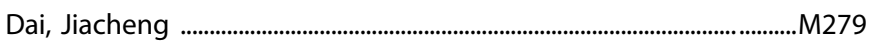

Dai, Jing ...............................................................................................................147

Dailey, Michael .............................................................................................. T258

Dalgard, Clifton .........................................................................................W108

Daly, Ella ........................................................................................................ M94

Daly, Rebecca .............................................................................................. T231

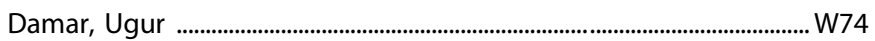

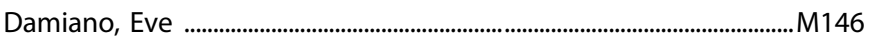

Dancy, Morgan M. ...................................................................................W138

Danforth, Alicia ................................................................................................. W120

D'Angelo, Heather ..............................................................................................T66

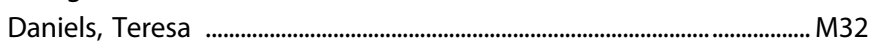

Darnley, Stefanie .............................................................................................. W263

Daroff, Rob ..................................................................................................... W120

Darrick, May ................................................................................................. W102

Daskalakis, Nikolaos ............................................................................ 11.5, T80

Daskalakis, Zafiris Jeff ...................................................................... M22, M191

Datta, Sandeep ...........................................................................................19.2

Davasaambuu, Sara ....................................................................................... M52

Davatzikos, Christos .............................................................227, W178, W202

David, Denis .....................................................................................T137, W107

David, Indira .........................................................................................T137, W107

Davidson, Michael ..................................................................................................W56

Davis, Brittany ..................................................................................................... M51

Davis, Ian ............................................................................................................. T4

Davis, John .......................................................................................T193, W180

Davis, Margaret ................................................................................. T25, W219

Davis, Robert .......................................................... T196, W123, W201, W203

Davis, Sarah ....................................................................................................W287

Davis, Shannon ............................................................................................ T111

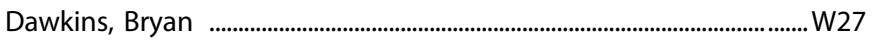

Day, Rebekah F. ........................................................................................ T130

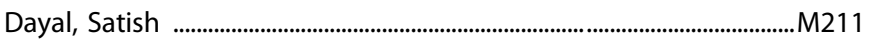

Dayan, Peter ................................................................................................... W105

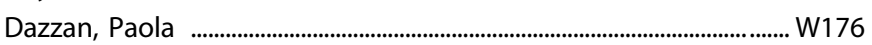

de Angelis, Paolo ............................................................................... T142, W89

De Baun, Heloise .............................................................................................. W199

De Bellis, Michael .................................................................................... M41, M58

De Biase, Lindsay ....................................................................................................2.

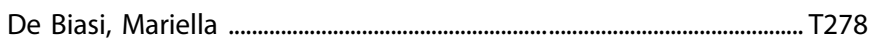

De Boer, Peter ....................................................................................................... W96

De Choudhury, Munmun .................................................................................. 38.7

De Corte, Benjamin ............................................................................................ M194

De Felice, Fernanda ........................................................................................... 2.2

De Filippis, Elena ........................................................................................... W137

de Goede, Annika .............................................................................................W74

De Gregorio, Danilo ............................................................................................ W153

de Guglielmo, Giordano .......................................................................... T264

De Haan, Lieuwe ..................................................................................................167

de la Fuente-Sandoval, Camilo ................................................................ W174

de Lecea, Luis ............................................................................................. 10, W223

De Luca, Vincenzo ...................................................................M184, T171, T217

de Quevedo, Joao ................................................................................. T143, W98

De Waal, Eric .............................................................................................W117

de Wit, Harriet .....................................................................M112, M223, M269

Dean, Douglas ........................................................................................................ T103

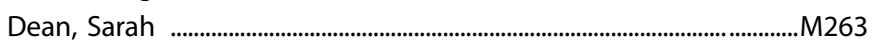

DeBattista, Charles ............................................................................................. T148

Debiec, Jacek .........................................................................................................W29

DeCastro, Alex ............................................................................................ W183

Deep-Soboslay, Amy .................................................................................. 1176

Defaix, Celine ................................................................................................. T137

DeFrancisco, Daniel ........................................................................................ W31

del Re, Elisabetta ......................................................................................M203

DelBello, Melissa ..............................................M23, T150, W91, W113, W114

Delcasso, Sebastien ........................................................................................... T86

Deligiannidis, Kristina ............................................................. T127, T138, W79

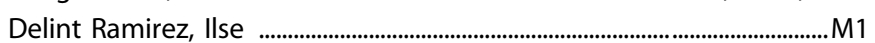


DellaGioia, Nicole

T25, W219

Dellarco, Danielle V. ............................................................................................... M12

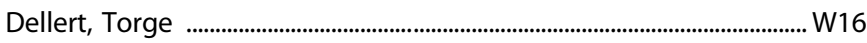

DeLorenzo, Christine ...............................................................W4, W97, W115

Deluisi, Joseph .............................................................................................W22

Demars, Michael ....................................................................................... T109

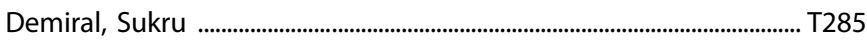

Demitrack, Mark ............................................................................................W142

Demjaha, Arsime ............................................................................................... W176

Demopulos, Christina ................................................................................. T124

Demos, Alexander ................................................................................................... T65

Demro, Caroline ................................................................................................. W179

Deng, Helen ........................................................................................................ T208

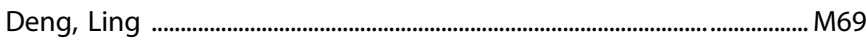

Deng, Zhi-De ...................................................................................... 58.4, M9

Dennery, Phyllis .............................................................................................. M32

Dennis, Cindy-Lee ........................................................................................... M28

Dennison, Jessica ................................................................................................W6

Denny, Christine Ann ........................................................................ 8.3, T137

Deonaraine, Kristina ......................................................................................... M13

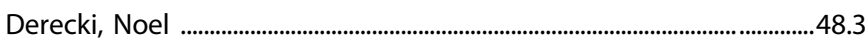

Dereschewitz, Eric .......................................................................................... T54

DeRosse, Pamela ....................................................................................... W169

Desai, Jinal ....................................................................................................... T90

Deshpande, Harshawardhan .................................................................... T252

D'Esposito, Mark ..............................................................................................M255

Desrochers, Stephanie .................................................................................... W73

Desta, Zeruesenay ................................................................................................. M23

Detke, Michael ...................................................................................................... 5.5

Deutsch, Stephen ............................................................................................. W44

Deutschmann, Andre ............................................................................... W259

DeVivo, Michael ............................................................................................. T230

Devlin, Bernie ...................................................................................................M204

Dew, Rachel ....................................................................................................... W260

DeYoung, Lena ......................................................................................287, W59

Dhamne, Sameer ...........................................................................................W45

Dhingra, Isha .................................................................................................. T1

Di Carlo, Pasquale .........................................................................................W209

Di Chiara, Gaetano .............................................................................T245, W249

Di Ciano, Patricia ..................................................................................................M237

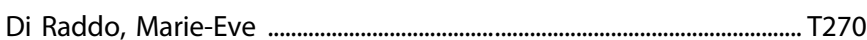

Diazgranados, Nancy .........................................................M246, T251, T256

Díaz-Zuluaga, Ana M. ................................................................................. W128

DiBernardo, Allitia ............................................................................................. T136

DiBerto, Jeffrey .......................................................................................................... T56

Dickerson, Faith ........................................................................................... M90

Dickie, Erin ...........................................................................M168, T92, W178

Dickinson, Dwight ................................................................................... T199

Dickstein, Daniel .....................................................................................287, W59

Didio, Kate .................................................................................................. T147

Didriksen, Michael ............................................................................................. W182

Dienel, Samuel .................................................................................................182

Dieterich, Andrew .......................................................................................... T140

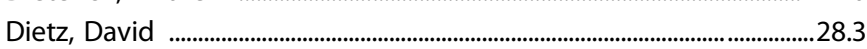

Dijkstra, Francis ........................................................................................................W74

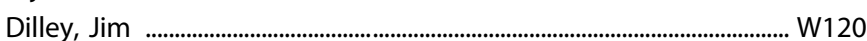

Dillon, Daniel .............................................................................................. T168

Ding, Ying ......................................................................................... M195, M204

Ding, Yu-Shin .............................................................................................. W214

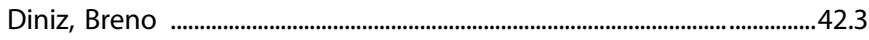

Dinka, Liban ............................................................................................... W184

Dion-Albert, Laurence ........................................................................... W109

Disney, Alex ..................................................................................................... W244

Diwadkar, Vaibhav .......................................................................................... T5

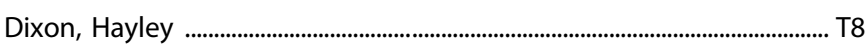

Dixon, Rushell ....................................................................................................M104

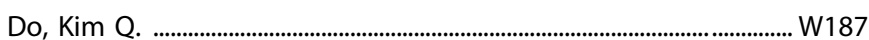

Do Monte, Fabricio ...............................................................................................W9

Dobbin, Josh ........................................................................................ T142, W89

Doblin, Rick ................................................................................................................39.2

Docherty, Anna ...............................................................................................28

Doenier, Emma .......................................................................................... T106

Doerig, Katherine ..........................................................................................T14

Doherty, James ...............................................M143, M144, M147, T127, W79

Dohi, Eisuke ................................................................................................ T190

Dolder, Patrick C ............................................................................................ W76

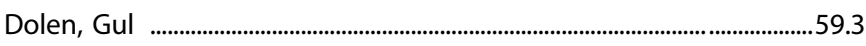

Domi, Esi .....................................................................................W238, W251

Dominick, Kelli .......................................................................................................... M4 M

Domino, Edward ......................................................................................................... W33

Donaldson, Zoe ..................................................................................................... T28

Donegan, Jennifer .............................................................................................. W228

Dong, Pengfei ........................................................................................................... T222

Dong, Tien ....................................................................................................... W140

Dong, Tony .................................................................................................W90

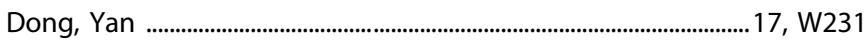

Dong, Zhe ................................................................................................. T263

Donohue, Meghan Rose ............................................................................M192

Donovick, Roger ....................................................................................... T247

Dorner-Ciossek, Cornelia ............................................................................W34

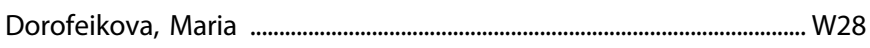

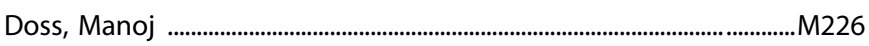

Doucette, Wilder ................................................................................. M170, T16

Douglas, Ella .................................................................................................. W65

Dourish, Colin ........................................................................................................W67

Dow, Holly ....................................................................................................................M213

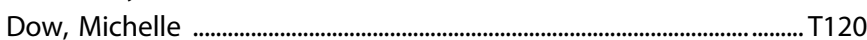

Dowling, Kevin ...................................................................................T63, W51

Doyle, Marie .......................................................................................M228, W224

Doyle, Michelle ......................................................................................................M236

Dreier, Allison ...................................................................................................... T278

Drevets, Wayne ...........................45, M86, M100, M108, T75, W117, W130

Drew, Michael ....................................................................................................... 8.2

Drummond, Katherine ...........................................................................T52

D'Souza, Deepak .................................................................T125, T276, W208

Du, Fei .................................................................................................... T22

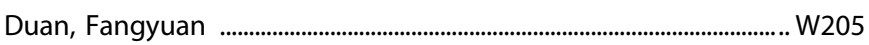

Duarte, Cristiane S. .......................................................................... T102, W49

DuBois, Jonathan .................................................................................. T269

Dudek, Katarzyna ................................................................................................. W109

Dudek, Serena ............................................................................................................ M79

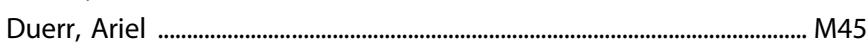


Duffecy, Jennifer ........................................................................................T65

Dufford, Alexander ........................................................................................... W60

Dugovic, Christine ......................................................................................W152

Dulawa, Stephanie ........................................................................................ W69

Duman, Ronald .............................................................................. T25, T125

Dumas, Julie .................................................................................................... T68

Dumitriu, Dani ................................................................................................... W57

Duncan, Erica ............................................................................................. T198

Dunlop, Boadie ....................................................................... T148, W92, W125

Dunner, David ................................................................................................ T124

Dunsmoor, Joseph .......................................................................................... M10

Duprat, Romain .................................................................................................W22

Durgam, Suresh ...................................................................W123, W201, W203

Durham, Leighton ......................................................................................... T168

Dûring, Signe .................................................................................................... W176

Durre, Reiley .............................................................................................. W222

Dutheil, Sophie .................................................................................................. T196

Duval, Elizabeth ............................................................................................... M11

Dwiel, Lucas ............................................................................................. M170, T16

Dwivedi, Yogesh ........................................................................................... T145

Dwork, Andrew J. ...................................................................................... W131

Dwyer, Jennifer ................................................................................................. M60

Dzemidzic, Mario ...........................................................................................M269

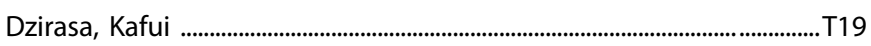

Dzombak, Jesse .............................................................................................. T10

Eack, Shaun

M183, M207

Earley, Willie ............................................................................................................ M99

Ebdrup, Bjørn H. ..................................................................................... W176

Eberhardt, Lauren .................................................................................................. M18

Eckert, William ................................................................................................... W152

Ecklund, Dixie ....................................................................................................M146

Edmiston, Kale .............................................................................................. M78

Edwards, Alexis ............................................................................................. T240

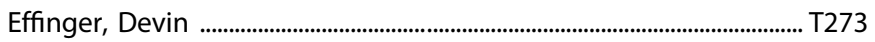

Egbert, Lizzy ............................................................................................ T105

Ehrman, Ronald ........................................................................................ W263

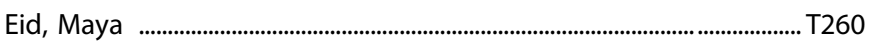

Einhorn, Nathan ................................................................................................ M77

Eisenberg, Daniel .........................................................M29, T199, W40, W211

Elam, Hannah ....................................................................................................... W228

Elkhatib Smidt, Stacey ..................................................................................... M213

Ellard, Kristen ...................................................................................M131, T175

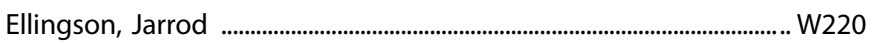

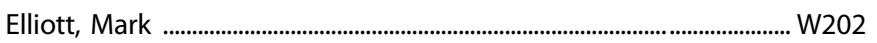

Elliott, Matthew ………………………………………………………………..... T113

Ellis, Steven P. .................................................................................................. M52

Elrahal, Farrah .....................................................................................131, T4

Elsaid, Nahla ............................................................................................. W122

Elsaid, Sonja ................................................................................................ M22

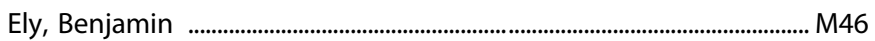

Ely, Timothy .....................................................................................T7, T98

Emery, Paul ....................................................................................................... T47

Emile, Arielle ..........................................................................................................M104

Engberg, Goran ...............................................................................M173, W197

Engelke, Douglas ....................................................................................................W

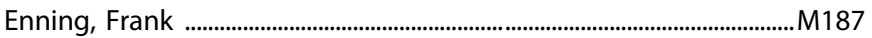

Enwright, John ...........................................................................................M180

Epperson, C. Neill ........................................................... 53.4, T31, T138, W60

Epstein, David .................................................................................W254, W256

Erb, Samuel .................................................................................................... W129

Erbes, Christopher ...................................................................................... T78

Erdman, Lauren ............................................................................................ T92

Erdmann-Gilman, Petra .............................................................................M234

Ereshefsky, Larry .............................................................................................. M72

Erhardt, Sophie ............................................................................... M173, W197

Erhart, Andrew ........................................................................................................ W60

Erica, Forbes ................................................................................................. W81

Erickson, Craig ...................................................................................... M40, W54

Erickson, Susan ..................................................................................................195

Eriksson, Hans .............................................................................................W111

Ernst, Monique ........................................................................... M61, T3, W17

Erus, Guray ....................................................................................................... M27

Eryilmaz, Hamdi ....................................................................................T63, W51

Escobar, Javier ......................................................................................... 30, 30.5

Eshel, Neir ....................................................................................................... M20

España, Rodrigo .........................................................................................M259

Espinoza, Randall ............................................................................... M81, T29

Esterlis, Irina ......................................................................... 12, M217, W219

Etherington, Sarah ........................................................................................... M85

Ethridge, Lauren ....................................................................................... M40, W54

Ethridge, Sarah ................................................................................................ W234

Etkin, Amit .................................................. 11.1, 58.1, M20, M89, T76, W99

Etropolski, Mila ................................................................................................... M86

Ettinger, Ulrich ................................................................................................W16

Eugenia Mesquita, Maria .............................................................................. T83

Eum, Seenae .................................................................................................... T37

Euston, Tanner .............................................................................................. W266

Evans, Jennifer .....................................................................T144, T161, W133

Evans, Travis C. .............................................................................................. $\mathrm{M} 12$

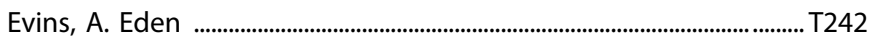

Ewin, Sarah ................................................................................................ T2 T2

Fadok, Jonathan ..................................................................................................W28

Fan, Mikayla ...................................................................................................... T151

Fan, Sili ......................................................................................................................... W19

Fan, Yan .......................................................................................................... W84

Fan, Yong ....................................................................................................... M27

Fang, Li Juan ................................................................................................. W180

Fanger, Christopher ....................................................................................... T22

Fani, Negar ..............................................................................................T7, T8, W53

Fanning, Jennifer .............................................................................................. T123

Farahi, Judah .................................................................................................... T247

Farahmand, Khodayar ............................................................................... T205

Farahmand, Khody ..................................................................................... T202

Farero, Ryan .............................................................................................................M240

Fargotstein, Molly ........................................................................................ T198

Farhat, Luis ................................................................................................. T34

Farinelli, Lisa ..............................................................................................T49

Farkas, Bence ................................................................................................... W143

Farley, Brandon ......................................................................................................M144

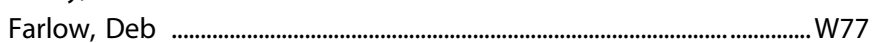


Farokhnia, Mehdi ........................................................................................ T49

Fatouros-Bergman, Helena ............................................................................ M173

Faucher, Christiana ....................................................................................... M21

Faulkner, Megan .................................................................................... M61, M62

Faulkner, Monica .......................................................................................... W2 W

Faunce, Kaycee ...............................................................................................245

Fava, Maurizio

M134, M161, W92, W125

Fazio, Leonardo

W209

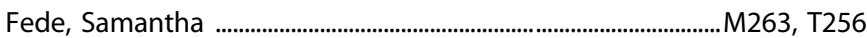

Feder, Adriana ...................................................................................................... T87

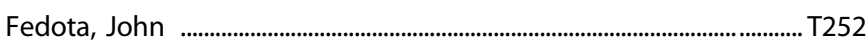

Feijo de Mello, Andrea ................................................................................ T102

Feijó Mello, Marcelo ................................................................................ T83, W8

Feindel, Kirk ................................................................................................... M85

Feinstein, Justin ........................................................................................... W27

Feja, Malte ......................................................................................................M257

Feld, Gordon .......................................................................................... 10.2, T44

Feldman, Dana ................................................................................................ 275

Felger, Jennifer ................................................................................ M135, W101

Felsing, Daniel ............................................................................................... M201

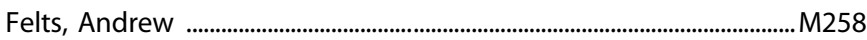

Fenchel, Daphna ............................................................................................ W56

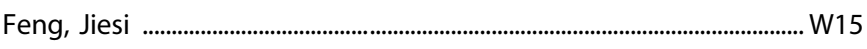

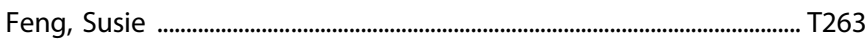

Feng, Ying-Hong ............................................................................................ M121

Fenske, Alicia ............................................................................................................ T181

Fenster, Robert ................................................................................................M8, T80

Ferguson, Laura ............................................................................................. T246

Ferguson, Susan ............................................................................... 23.2, W222

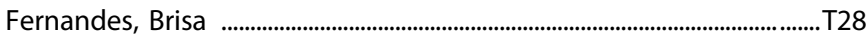

Fernandez, Diego .......................................................................................... M33

Fernandez, Thomas ............................................................................ T34, W38

Fernandez-Leon, Jose ...................................................................................W9

Ferrante, Michele ..................................................................................................38.6

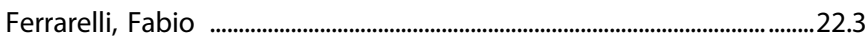

Ferrer Perez, Carmen ................................................................................. W109

Ferris, Anjali ................................................................................................ T142

Ferris, Mark ............................................................................................................. W264

Feusner, Jamie ............................................................................22.1, T13, W158

Fichorova, Raina ............................................................................................. T162

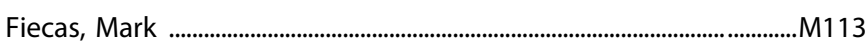

Fiedorowicz, Jess G. ...................................................................................... T148

Fiehn, Oliver ......................................................................................................... W19

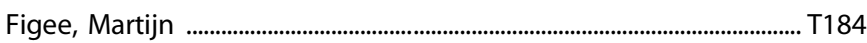

Filbey, Francesca ................................................................................ M216, M221

Filippou-Frye, Maria ....................................................................................... T74

Filippov, Gleb ............................................................................................... M211

Findling, Robert .......................................................................................... T134

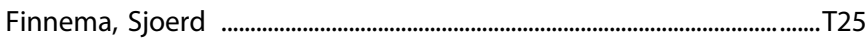

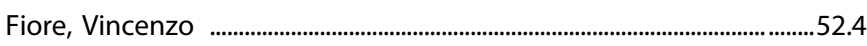

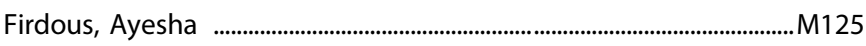

Fischer, Adina ..................................................................................127, W94

Fish, Kenneth ......................................................................M182, T228, W195

Fisher, Miranda ............................................................................................. T254

Fisher, Patrick M. ................................................................................................... T177

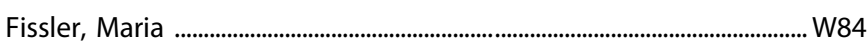

Flanagan, John M71

Flanigan, Meghan

$\mathrm{M} 13, \mathrm{~T} 89, \mathrm{~W} 282$

Flavin, Karen

M196

Fleck, David

W91, W114

Fleischer, Leah

.. W114

Fletcher, Paul

M38, T225

Flint, Alastair

M130, W178

Flores, Cecilia

Floresco, Stan

$44.3, \mathrm{~T} 234$

Flores-Ramirez, Francisco ............................................................................... T128

Flory, Janine ........................................................................................................ T282

Flowers II, James .................................................................................................. T234

Foerster, Bernd Uwe ...........................................................................................W8

Foley, Andrew .................................................................................................. W143

Folorunso, Oluwarotimi ..................................................................T173, W193

Fonzo, Gregory ..............................................................................................T76

Foran, William ..............................................................................................172

Forbes, Erika ............................................................................. M62, M78, T110

Ford, James ............................................................................................... W122

Ford, Judith ......................................................................................... T214, T218

Forester, Brent P. ......................................................................................... T148

Formica, Richard ........................................................................................ T125

Forsberg, Christopher ................................................................................ W108

Forsyth, Ashleigh ................................................................................................W14

Forsyth, Jennifer ...................................................................................................T12

Foss-Feig, Jennifer .................................................................................. 51.4, M192

Fossler, Michael ..................................................................................................... W142

Fouda, Samar ..........................................................................................................M107

Fourgeaud, Lawrence .................................................................................... T139

Fournier, Jay ........................................................................................... M78, T283

Fournier, Margot ........................................................................................ W187

Foussias, George .............................................................................. T194, T225

Fowler, Christie ...........................................................................................150

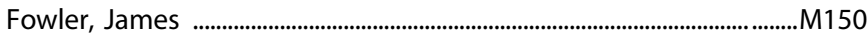

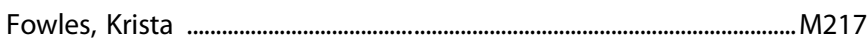

Fox, James B. ............................................................................................ W138

Fox, Peter T. ................................................................................................ M48

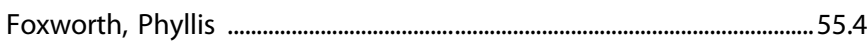

Fraiser, Claire M. .............................................................................................. T272

France, Charles .................................................................................................. W244

Francis, Sunday ................................................................................................ T10

Franco, Olivia ..................................................................................................... W200

Frank, Ellen .............................................................................. 55.1, 55.7, W134

Frank, Guido ............................................................................................22, 27.4

Frank, Josef ...................................................................................................... W2 W

Franklin, Teresa ................................................................................................ W263

Franklin, Tina ............................................................................................... T163

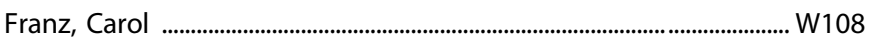

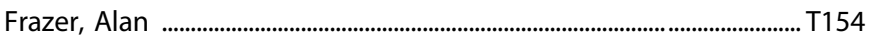

Frazier, C. Jason .........................................................................................W70

Frazier, Thomas ................................................................................................ T134

Fredriksson, Ida ............................................................................... 17.4, M232

Free, R. Benjamin ......................................................................... M162, W181

Freedman, Robert ...................................................................... 43, 43.1, M164

Freeman, Brandi ......................................................................................... W57

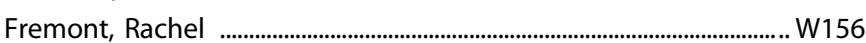




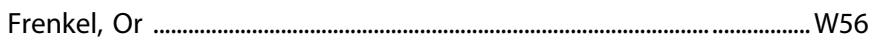

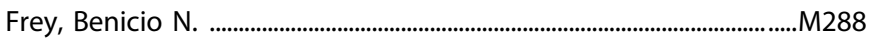

Freyberg, Zachary ........................................................................................M162

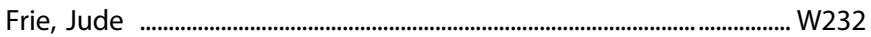

Fried, Eiko ......................................................................................................W13

Friedman, Allyson ............................................................................. 23.3, T89

Friedman, Jeffrey ...........................................................................................T14

Friend, Samantha .........................................................................................W32

Fries, Gabriel ......................................................................... T143, W98, W237

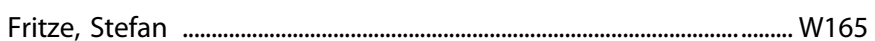

Frohlich, Flavio ..................................................................................................... T286

Frokjaer, Vibe ........................................................................................ W121

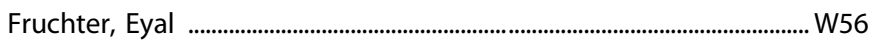

Fry, Megan ..................................................................................................... T23

Frye, Mark ...............................................................................................M120, W137

Fryer, Susanna ......................................................................................... T214

Fu, Dong-Jing .......................................................................................M94, W130

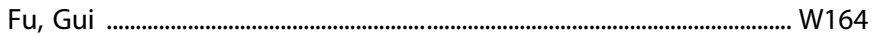

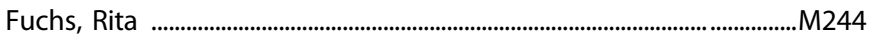

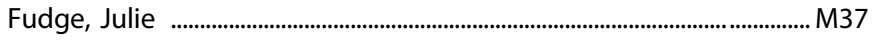

Fuerst, Lauren .............................................................................................. W231

Fugate, Ryan ............................................................................................. T124

Fujii, Shinya ................................................................................................... W162

Fukuda, Andrew .................................................................................................T167

Fulenwider, Hannah ............................................................................................. M1 16

Full, Jan ...................................................................................................................... T18

Fullard, John ............................................................................................ T222

Funes, Christopher ....................................................................................... M131

Funke, Jonathan ........................................................................................M225

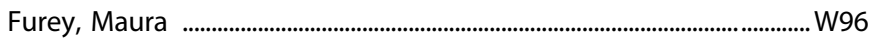

Furuyashiki, Tomoyuki ............................................................................... M82

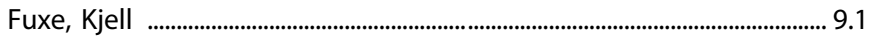

Gabbay, Vilma

29.4, M46

Gajewski-Kurdziel, Paula

T186

Galante, Allison

...T57

Galatzer Levy, Isaac

..11.2, T223

Galbraith, Jordan

T132

Galderisi, Silvana

Gallagher, Keith

W176

W179

Gallego, Juan

30.3, T197

Gallezot, Jean-Dominique

M218, T25

Gallo, Eduardo .................................................................................................... T6

Gallop, Danisha ................................................................................................ M51

Galynker, lgor ...............................................................................................W3

Gama, Clarissa ....................................................................................................185

Gandhi, Zeal H ................................................................................................ T198

Gangisetty, Omkaram .............................................................................. T261

Gantz, Stephanie ....................................................................................... W250

Gao, Mei Hua .....................................................................................................W32

Gao, Qingzhu ............................................................................................. W1

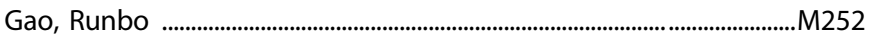

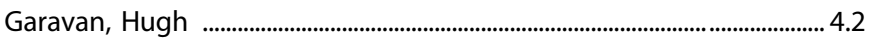

Garcia, Aaron ..................................................................................................... W222

Garcia, Benjamin ............................................................................T234, W221

Garcia, Erik ............................................................................................................... M238

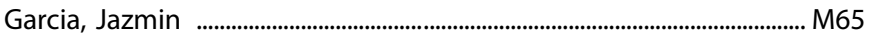

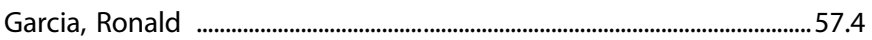

García-Alcon, Alicia .....................................................................................W42

Garden, Gwenn .......................................................................................... T265

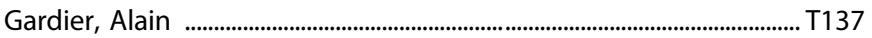

Gardner, Margaret ....................................................................................... T221

Garibay, Nancy ............................................................................................ W230

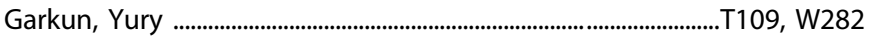

Garnaat, Sarah ......................................................................................................... M21

Garrison, Aaron .................................................................................................... W159

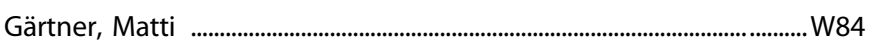

Garvin, Mona ......................................................................................................... T181

Garza, Kristie .................................................................................................... T163

Gaspar, Pablo ...................................................................................... M286, T41

Gass, Justin T. ............................................................................................M247

Gass, Natalia ............................................................................................... W182

Gassen, Nils ...................................................................................................... T224

Gatta, Eleonora ............................................................................................ W276

Gau, Susan Shur-Fen ......................................................................... M39, W47

Gaulden, Andrew ...........................................................................................225

Gawrysiak, Michael ................................................................................... W263

Gee, Dylan ....................................................................................................... T94

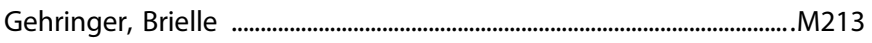

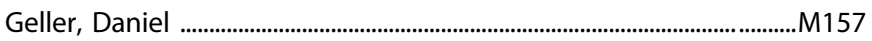

Gemo, llaria ....................................................................................................... W145

Gendy, Marie ...............................................................................................................M237

George, Mark S. ........................................................................................ W138

George, Olivier .................................................................................M255, T264

George, Susan .................................................................................. T266, T270

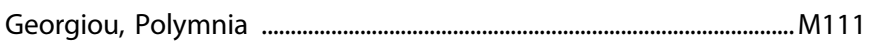

Gerak, Lisa ............................................................................................... W24

Geramita, Matthew ...................................................................................... W194

Gerard, Sanacora .......................................................................................... T25

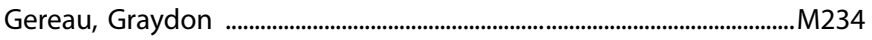

Gerhardt, Greg ...........................................................................................M219

German, J. Bruce ........................................................................................... T120

Gershon, Elliot ............................................................................................... T37

Geske, Jennifer ..............................................................................W265, W272

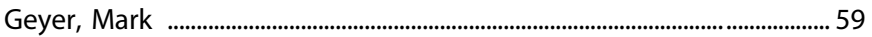

Ghahremani, Dara ................................................................................... T23, T247

Ghate, Pankaj ........................................................................................................... T111

Ghay, Sahil ................................................................................................... T2

Gheidi, Ali ........................................................................................................M284

Gholam-Reezaee, Mehdi ............................................................................. W187

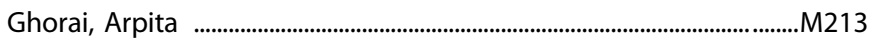

Ghoshal, Soham .......................................................................................... W248

Giacometti, Laura ............................................................................................ T235

Giardino, William ......................................................................................... W223

Giasson, Giasson ...................................................................................... T2

Gibson, Abigail ............................................................................................... W234

Gibson, Amanda ................................................................................................W2 W

Gibson, Laurel ..........................................................................................W220

Gilbert, Anna .......................................................................................287, W59

Gildawie, Kelsea ................................................................................................. T91

Gill, Mary Kay ...................................................................................W103, W135

Gillespie, Charles ................................................................................................. T8 
Gilmore, Casey

..T181, W268

Gilpin, Nicholas

$40,40.2$

Ginder, Nathaniel

.. $\mathrm{T} 247$

Giorgi, Salvatore

W200

Giorgi Manin, Marina

T102

Gipson-Reichardt, Cassandra

.. W218

Girdhar, Kiran

T222

Girgis, Ragy

. T219

Gladden, Catherine

Glahn, David

...146

Glaser, Paul M48, M167, T131

Glausier, Jill M219

Gleason, Kelly

M180, W241

Glegola, Justyna

.....T24

Glenthoj, Birte

...M174

Glimcher, Paul

W176

Glover, Matthew ..........................................................................................................W87

Gluck, Rachel .................................................................................................... T8

Gnecco, Tamara ............................................................................................ W61

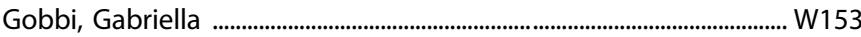

Godbout, Jonathan .......................................................................................... 48.1

Godwood, Alex ...........................................................................................181

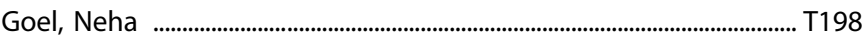

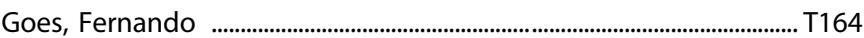

Goff, Allison .............................................................................................. M97, T166

Gogos, Joseph .......................................................................................................... M198

Goktas, Selin ................................................................................................. T125

Golay, Philippe ............................................................................................... W187

Gold, James ....................................................................................... M160, W179

Gold, Michael .................................................................................................... W104

Goldberg, Jack ...........................................................................................W108

Golden, Sam ................................................................................................... W109

Goldenberg, Anna .......................................................................................T92

Goldenthal, Ariel ........................................................................................... T153

Goldman, David ............................................ M97, M246, T166, T236, W272

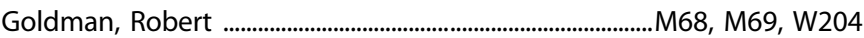

Goldsmith, David ...........................................................................T198, W101

Goldstein, Benjamin ...................................................................................W93

Goldstein, Jill ....................................................................................57, 57.3, M3

Goldstein, Rita ................................................................................T204, W277

Goldstein, Tina ...................................................................................W103, W135

Goldstein-Piekarski, Andrea ........................................................... T26, W132

Gomes, Felipe .............................................................................................. M43

Gomez, Charlyn ....................................................................................................... M44

Gomez, Juan ............................................................M232, W141, W246, W271

Gonçalves, Vanessa .......................................................................................T35

Gong, Qiyong ...................................................................... M156, W163, W164

Goniewicz, Maciej .............................................................................................222

Gonzalez, Cassandra .................................................................................. T28

Gonzalez-Peñas, Javier ...............................................................................W42

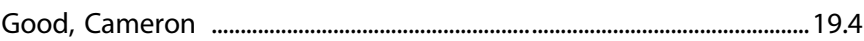

Goodkind, Madeleine ................................................................................T76

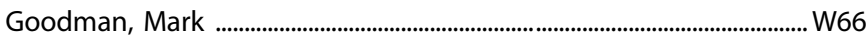

Goodwin, Shelby ................................................................................................ T60

Gordon, Andrew ........................................................................................ T113

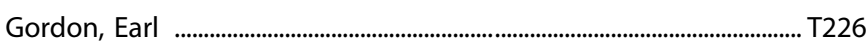

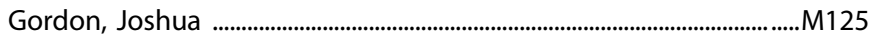

Gorelick, David ..................................................................................................M160

Gorelik, Aaron ..................................................................................................127

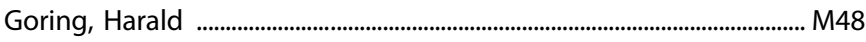

Gorka, Stephanie ................................................................................ M269, T30

Gosnell, Savannah .......................................................................................107

Gotlib, Ian ................................................................................................... W94

Gould, Robert .......................................................................................... M258, W159

Gould, Todd

$\mathrm{M} 111, \mathrm{~T} 137, \mathrm{~T} 161$

Gowin, Joshua

T249, T251, W261

Grace, Anthony ................................................................................... M43, M114

Grace, Peter .................................................................................................................T66

Grace, Woodruff ................................................................................................W78

Graff-Guerrero, Ariel ......................................................................M64, W162

Graham, James ............................................................................................ 161

Granata, Lauren ..............................................................................................T91

Grannemann, Bruce ..................................................................................... M134

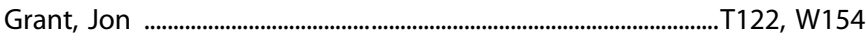

Grant, Steven ............................................................................................................ 4

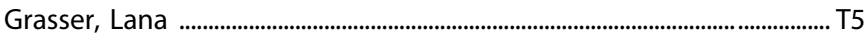

Grassie, Hannah ............................................................................................. M96

Grassi-Oliveira, Rodrigo ............................................................................ W237

Grasso, Jesse ........................................................................................................ T204

Graur, Simona .................................................................... M78, T233, T283

Grayson, Dennis ..............................................................................T271, W276

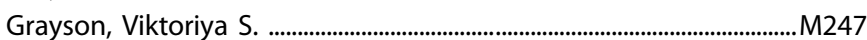

Graziane, Nicholas ............................................................................................ W231

Greally, John ....................................................................................................... T6

Greden, John ................................................................................. T148, M87

Green, Alan .............................................................................................. M170, T16

Green, Michael ............................................................ M165, T203, T209, T210

Green, ReJoyce ............................................................................................M261

Greenberg, Benjamin ............................................................ M158, T64, T70

Greenberg, Tsafrir ................................................................. M78, T233, T283

Greene, Jonathan ............................................................................................ M108

Greenfield, Ariele .............................................................................................. W207

Greenwood, Tiffany .................................................................................... T210

Greer, Tracy ..................................................................................................... T156

Gregory, Michael ..........................................................M29, M283, T199, W40

Gregory-Flores, Adriana ................................................................................. T272

Grice, Dorothy ............................................................................................47.1, W21

Griffiths, Roland ......................................................................59.1, M226, W102

Griffiths, Zoe ....................................................................................................M213

Grigoleit, Jan-sebastian ........................................................................... T139

Grigoriadis, Sophie ............................................................................................. M28

Grillon, Christian ..........................................................M9, T3, T31, T241, W17

Grim, Travis W. .............................................................................................. T268

Grimm, Simone .....................................................................................W84

Grinband, Jack .............................................................................. M164, W199

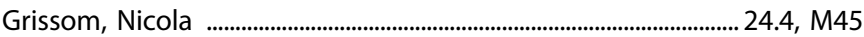

Grodin, Erica ........................................................................................ M253, M261

Grogans, Shannon ..............................................................................M29, W211

Groll, Dianne .....................................................................................................W14

Groman, Stephanie ..............................................................................................M217

group, ALSPAC ……………………….................................................... T240

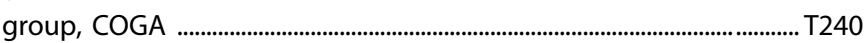


group, Generation Scotland ................................................................... T240

Grubisha, Melanie ..............................................................................................M195

Gruener, Daniel .............................................................................................. T147

Grunfeld, Jennifer ............................................................................................ T75

Gryglewski, Gregor ..................................................................................... W127

Grzenda, Adrienne ..........................................................................................T74

Guay-Ross, Robert N. ....................................................................................W5

Gueorguieva, Ralitza ......................................................................T125, W272

Guerin, Alexandre ..................................................................................... T52

Guest, Paul .......................................................................................................... M166

Guevara, Christopher ..................................................................................... M13

Guglin, Anthony ........................................................................................M265

Guidotti, Alessandro ........................................................................T193, W276

Guilloux, Jean-Philippe ...............................................................T137, W107

Gumbert, Andrew ...................................................................................... T266

Gunduz-Bruce, Handan .................................................................. T127, W79

Gunter, Barak .................................................................................................... W159

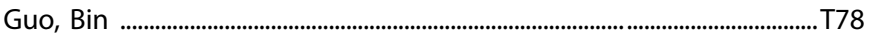

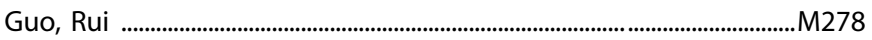

Gupta, Ruchi .........................................................................................W137

Gur, Raquel ................... M27, M48, M210, T93, T131, T210, W200, W202

Gur, Ruben .............................................M27, M210, T93, T131, T210, W202

Gur, Tamar .............................................................................................. W37

Gurnani, Sarika ..................................................................................................W45

Gurney, Mark ................................................................................................ W151

Gust, Charleen ........................................................................................................ W220

Gutmann, Laurie ........................................................................................ T182

Gysling, Katia ..........................................................................................................M273

Haass-Koffler, Carolina ........................................................................ M276, T51

Haber, Suzanne .................................................................................................M158

Hacker, Marcus ................................................................................................ W127

Hadas, Okon-Singer .................................................................................... W81

Hafeman, Danella ............................................................................. T135, T283

Hafizi, Sina ....................................................................................T36, T237

Hafner, Kathrin ........................................................................................ T224

Hagan, Kelsey ....................................................................................M127, W94

Hahn, Britta ..............................................................................................281

Hahn, Emily ........................................................................................................ M19

Hahn, Margaret ........................................................................................ M64, T211

Haile, Meron ..................................................................................................... T45

Haj-Dahmane, Samir .................................................................................. M257

Hajeissa, Alaa ...................................................................................................... T24

Hakimi, Michael .................................................................................................... M84

Halaris, Angelos .................................................................................M84, W139

Halberstadter, Kimberly ............................................................................. T278

Hall, Mei-Hua ....................................................................................... M197, M209

Hall, Nancy ......................................................................................................... M211

Haller, Simone .................................................................. M56, M96, T84, W13

Hallin, Michael ..............................................................................................M194

Hallschmid, Manfred ..............................................................................T44, W67

Halverson, Hunter .............................................................................................M194

Hamborg, Madeline .................................................................................... W40

Hamidullah, Shahnaza ................................................................................ W232

Hamilton, Derek .................................................................................................... M49

Hamilton, Holly .............................................................................................. T2
Hamilton, Paul

T250

Hamilton, Peter ........................................................................56.3, T52, W231

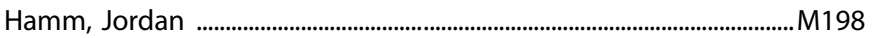

Hammond, H. Kirk ...........................................................................................W32

Hamoud, Abdul ......................................................................................... W177

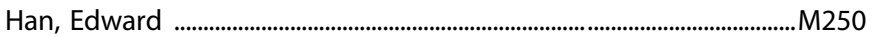

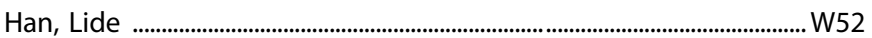

Han, Ming-Hu .................................................................................................... T89

Han, Seungwook ……………………………………….............................. M53

Han, Xiaoyan ...................................................................................................

Handa, Robert .................................................................................................. 57.2

Handschuh, Patricia ........................................................................................ W127

Haney, Margaret ...................................................................................... T33, T262

Hanford, Lindsay C. ......................................................................................... T99

Hankus, Allison ...................................................................................................... T198

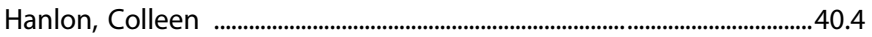

Hanna, Lauren .................................................................................................... W90

Hannett, Katrina ............................................................................................. M52

Hanratty, Lynsey .......................................................................................... W143

Hansen, Adam E. .............................................................................................. T177

Hansen, Hanne ...................................................................................................... T177

Hanson, Elizabeth ........................................................................................

Hantsoo, Liisa ................................................................................................. T31

Hao, Ke .................................................................................................................. M13

Hardee, Jillian ....................................................................................W235, W240

Harden, Scott .................................................................................................... W70

Harder, Jessica ................................................................................................ T162

Hareli, Maya ................................................................................................. T242

Harlalka, Suyash ...............................................................................................M250

Harnett, Nathaniel ............................................................................................ T7

Haroon, Ebrahim ........................................................................135, W101

Harper, Cierra ..................................................................................................... M95

Harper, Jessica ........................................................................................... T119

Harrington, Anthony ....................................................................... W78, W100

Harris, Adron .............................................................................................. T246

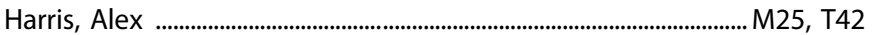

Harris, Alexander ...................................................................................................

Harris, Sion .................................................................................................................. M49

Hartmann, Jakob ...........................................................................M79, T80

Harvey, Meredith .....................................................................................................T76

Harvey, Philip ..............................................................................................T207, W2

Harvey, Theresa ......................................................................................... W193

Hasbi, Ahmed ............................................................................................... T270

Hashim, Eyesha ..................................................................................................... M64

Hashimie, Jaffrey ......................................................................................... T149

Hashimoto, Kenji ..........................................................................................W95

Hasler, Gregor .....................................................................................................W83

Haswell, Courtney .......................................................................... T85, W287

Hatano, Mai ..................................................................................T133, W226

Hattar, Samer ................................................................................................... M33

Hauser, Sheketha ................................................................................................ T280

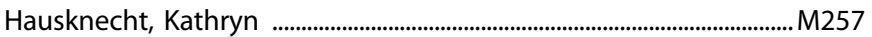

Haussmann, Sebastien ............................................................................ W144

Hawco, Colin ................................................................................................. M191

Hawken, Emily ................................................................................................... W14

Hayes, Matthew …………………………………………………………………....M270 
Haynes, Heather ........................................................................................... T279

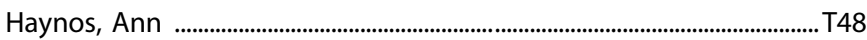

Hayward, Marshall A. ......................................................................................W6

Heal, David .................................................................................................... W215

Heckers, Stephan ........................................................................................ W166

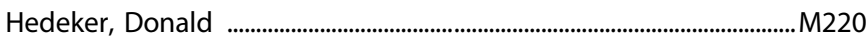

Hegarty, Catherine ...................................................................................... W211

Hegde, Rachal ............................................................................................. T117

Heilig, Markus ................................................ T241, T250, W229, W238, W251

Heimer-McGinn, Victoria ....................................................................... 15.1, 15.2

Heinsbroek, Jasper .............................................................................................. T54

Heishman, Stephen ................................................................................... M237

Heitzeg, Mary ........................................................................W235, W236, W240

Hellbusch, Brandon .......................................................................................... T2

Hellemann, Gerhard ........................................................................................ M81

Heller, Elizabeth .................................................................................................... 56.1

Hellmich, Martin ................................................................................................M187

Helmer, Cassandra ........................................................................................ M195

Hen, Rene ..........................................................................................M25, W131

Hencic, Boris .................................................................................................... W212

Hendricks, Ashley ..................................................................................... T279

Hendrickson, Rebecca ................................................................................. W25

Hendrickson, Timothy ...................................................... M113, T181, W268

Henkel, Nicholas ................................................................................................. W177

Hennessy, Lauren ........................................................................................................ M85

Hennig, Juergen ........................................................................................................... W16

Hennings, Augustin ................................................................................................... M10

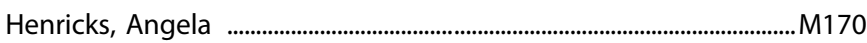

Hensler, Christopher .....................................................................................2204

Herbstman, Julie ......................................................................................... T107

Herman, Gabrielle ................................................................................................... M168

Herman, Melissa ............................................................................................M229

Hernandez, Caesar ................................................................... M4, W72, W255

Hernandez, Edith ..................................................................................... T18

Hernandez, Giovanni ................................................................................... T53

Hernandez, Jose Cesar .............................................................................. T172

Hernandez, Michael J. ........................................................................... T149

Herringa, Ryan ..................................................................................... M19, T72

Herrmann, Evan .................................................................................................. T262

Hersch, Steven ............................................................................................................M146

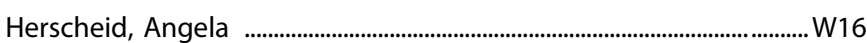

Heslin, Kesley ........................................................................................................ M194

Hess, Kathryn ............................................................................................... W187

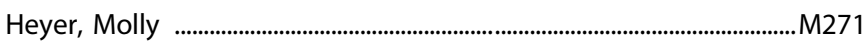

Heyn, Sara .........................................................................................................T72

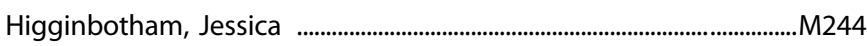

Higgins, Amy .............................................................................................M197

Higgs, Suzanne ..............................................................................................W67

Highland, Jaclyn ......................................................................................... T137

Hild, Allison ...................................................................................................W6

Hildebrand, Mariah .............................................................................M18, W241

Hill, Elizabeth ................................................................................................T11

Hill, Matthew ..................................................................................................... 24, T282

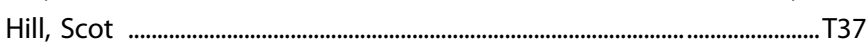

Hillard, Cecilia ............................................................................................. T58, W30

Hillmer, Ansel ..................................................... M217, M218, M241, T47, W10

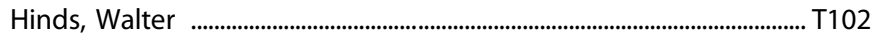

Hinrichs, Rebecca ..............................................................................................W23

Hipolito Nitzsche, Samara ............................................................................T83

Hipwell, Alison .............................................................................................. T99

Hirano, Yoji .....................................................................................................

Hirjak, Dusan ............................................................................................ W165

Ho, Shaun ......................................................................................................... W269

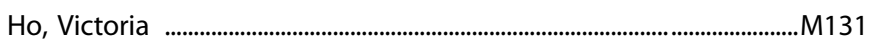

Hoag, Steven .......................................................................................................M160

Hochberger, William ....................................................................................M210

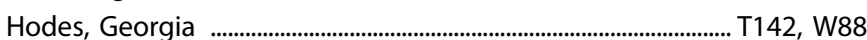

Hodgkins, Paul ...................................................................................................... T127

Hodgkinson, Colin ......................................................................................... W272

Hoeppner, Daniel ...............................................................................M176, T206

Hoexter, Marcelo .................................................................................................M138

Hoffman, Ellen ....................................................................................................... 36.2

Hoffman, Gabriel ............................................................................. T222, W52

Hoffman, Michaela .......................................................................................... M251

Hoffmann, Elis Vivianne ............................................................................ T102

Hofford, Rebecca ......................................................................................... W266

Hoftman, Gil .......................................................................................M48, W184

Hogeveen, Jeremy ...................................................................................... T113

Holden, Daniel ...............................................................................................M217

Holderness, Eben ............................................................................................M209

Holm, Lovisa ................................................................................................................ W251

Holmes, Karima .......................................................................................................M277

Holmes, Sophie ...................................................................................... T25, W219

Holmes, Traci ........................................................................................................ M63

Holsen, Laura ........................................................................................... M5, T168

Holt, Emma ............................................................................................. M125, T42

Holter, Michael ...................................................................................................... W218

Holtz, Nathan ................................................................................................M240

Holtzheimer, Paul .................................................................................... W122

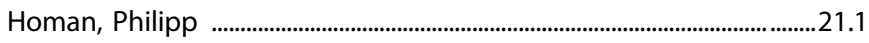

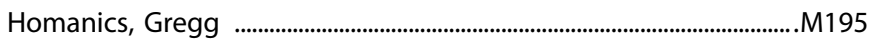

Hommel, Jonathan ........................................................................ M152, W253

Honda, Shiori ............................................................................................... W162

Honda, Sokichi ....................................................................................................... T195

Hong, Elliot ........................................................................................M128, W112

Hong, Sa-lk ....................................................................................................... W217

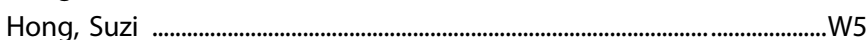

Honrao, Chandrashekhar ................................................................................ W31

Hook, Roxanne .............................................................................................. T122

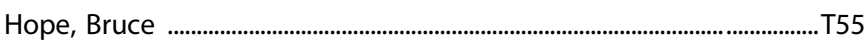

Hopkins, Jordan .............................................................................................M230

Hopkins, Seth .......................................................................................T215, W204

Hopkinson, Casey .......................................................................................... W51

Hoptman, Matthew ................................................................................ W178

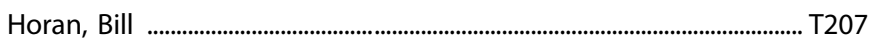

Horan, William P. ................................................................................... T209

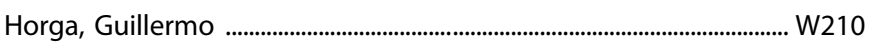

Hough, David ............................................................................................. W130

Houle, Sylvain ....................................................................................T36, W62

Howard, Christopher .......................................................................................... T11

Howes, Oliver ................................................................................21.4, M174, M190

Hsieh, Cheng-Yu ...................................................................................................... M39 
Hsieh, Jenny .................................................................................................. W228

Hsieh, Lawrence .................................................................................................. T9

Hsiung, Abigail .................................................................................................. T3

Hsu, David ........................................................................................................

$\mathrm{Hu}, \mathrm{Na} \quad$............................................................................................................ W164

$\mathrm{Hu}$, Ruifeng ..............................................................................................W237

$\mathrm{Hu}$, Yang ........................................................................................................ M67

Huang, Guoping ..............................................................................W163, W164

Huang, Ming-Yi ............................................................................................ T127

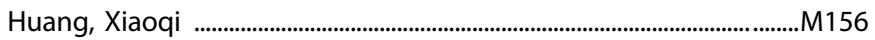

Huang, Yanhua ............................................................................................ W231

Huang, Yiyun ...................................................M218, M241, T47, T276, W10

Huang, Yung-Yu .............................................................................................. T153

Huber, Kimberly ................................................................................ 16.4, M278

Huebschman, Jessica .................................................................................. M54

Huettl, Peter .................................................................................................M219

Huey, Edward .................................................................................................... W156

Hughes, Dalton ...............................................................................................T19

Hughes, Dylan ....................................................................................T63, W51

Hughes, Thomas ………………………………………………........ M93, T147

Hulshoff Pol, Hilleke .......................................................................................2.26.2

Hultman, Christina M. .................................................................................W21

Humble, Jasmin ................................................................................165, T209

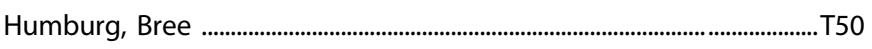

Hunker, Avery .................................................................................................M265

Hunt, Bethany .............................................................................................. T1

Huntenburg, Julia ................................................................................................ W84

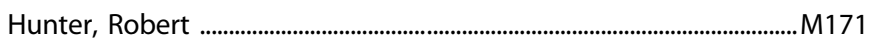

Hunter, Sarah .............................................................................................. M129

Huntington, Franklin .................................................................................... T63

Hupalo, Daniel .............................................................................................. W108

Huppert, Theodore ....................................................................................... T1 10

Hurd, Yasmin .................................................................................................W31

Hurlemann, Rene ..........................................................................................W16

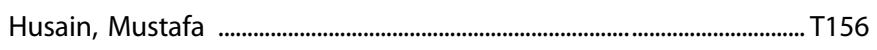

Husbands, Stephen ................................................................................. W244

Hussain, Faraz ..............................................................................................T65

Hutchison, Kent ...........................................................................W220, W243

Hutten, Nadia RPW ....................................................................................W76

Huynh, Trung .................................................................................................... W207

Huys, Quentin ...................................................................................31.2, W86

Hyde, Thomas ......................................................... M175, M176, M199, T224

Hye Cheon, Seon ........................................................................................... T111

ladarola, Michael .......................................................................................................M141

ladipaolo, Allesandra ..........................................................................M31, T4

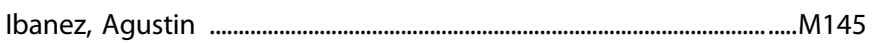

Ibeschitz, Harald ........................................................................................W127

Ide, Jaime ............................................................................................................. M36

Ikai-Tani, Saeko ...........................................................................................W168

Im, Susanna .........................................................................................T109, W282

Imbeault, Sophie ...................................................................................... W197

Indriolo, Teresa .......................................................................................... T242

Ingelman-Sundberg, Magnus .................................................................M117

Iñiguez, Sergio ................................................................................................ T128

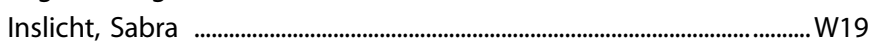

Investigators, COGS .......................................................................................... M210

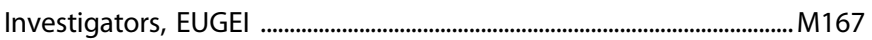

Investigators, GROUP ...............................................................................M167

lonescu, Dawn ...................................................................................M94, W130

losif, Ana-Maria ........................................................................................... W185

Irina, Esterlis .................................................................................T25, W10

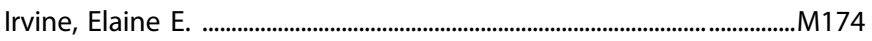

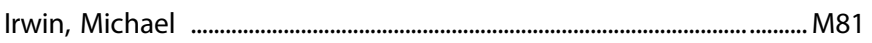

Isltam, Alvi ......................................................................................................W93

Itzhaky, Liat ...................................................................................................... M52

Itzkowitz, Debra …………………………………………………………………....... 146

Ivanov, Iliyan ..................................................................................................... M59

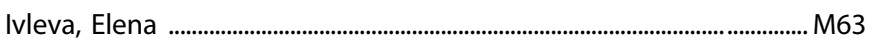

lyengar, Satish ..................................................................................... M207, W103

lyengar, Smriti .................................................................................................. T176

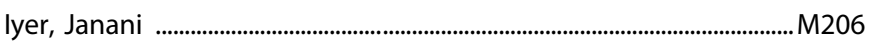

lyer, Kartik ........................................................................................................ W231

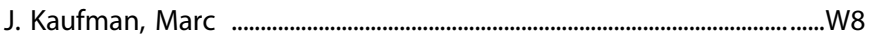

Jablensky, Assen .......................................................................................... T131

Jablonski, Michael R. ................................................................................... T148

Jackowski, Andrea .............................................................................................. T102

Jackson-Leyrer, Jonna .............................................................................. W218

Jacky, Debora ....................................................................................................... T86

Jacob, Suma .................................................................................................... T10

Jacobs, Emily ................................................................................................................. M3

Jacobs, Gabriel .......................................................................................................... W74

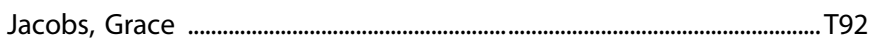

Jacobs, Jonathan ......................................................................................... W140

Jacobsen, Paula .................................................................................................M103

Jacobson, Moriah ............................................................................. M121, M137

Jaffe, Andrew .......................................18.1, M51, M175, M176, M199, T224

Jaffe, Jacob .........................................................................................................

Jagannathan, Kanchana .............................................................. M266, W263

Jain, Manish ...................................................................................................M201

James, Anthony ..........................................................................W167, W170

James, George ...................................................................................M19, W115

James, lan ..................................................................................................... W142

James, Sturgill ............................................................................................. W173

Kasper, James ..................................................................................... M152, W253

Jancic, Jasna ........................................................................................................ W212

Janczura, Karolina ...............................................................................W6, W7

Janda, Kim .................................................................................................M245

Janecka, Magdalena ......................................................................................W21

Janis, Reinelt ...................................................................................................W81

Jaquish, Abigail ........................................................................................... T264

Jarcho, Johanna ...............................................................................................105

Jaric, Ivana ..................................................................................................... T6

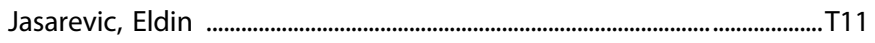

Jasna, Hocevar-Trnka .................................................................................. W192

Jastreboff, Ania ...............................................................................................M270

Javanbakht, Arash ....................................................................................... T5

Javitch, Jonathan ........................................................................................W69

Javitt, Daniel .....................................................51.1, 51.5, M164, M286, W199

Jaworska, Natalia .............................................................................................. T238

Jedema, Hank .......................................................................................T273, W141 


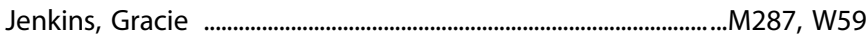

Jenkins, Lisanne ............................................................................................... T30

Jenkins, Paul ................................................................................................... T165

Jenni, Raoul ................................................................................................. W187

Jennifer, Schroeder ................................................................................... W256

Jeong, Isaac H. ..........................................................................................W12

Jeske, Claire ....................................................................................................W60

Jeste, Dilip

38, T227, T231

Jeste, Shafali ........................................................................................................ 51.2

Jeyachandra, Jerrold ...................................................................................M168

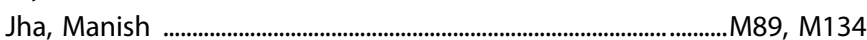

Jhou, Thomas ................................................................................................ T260

Ji, Gang .............................................................................................................. M67

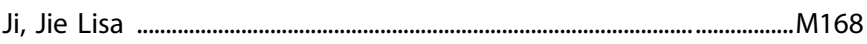

Jiang, Danni .................................................................................................. T52

Jiang, Janina ................................................................................................... M81

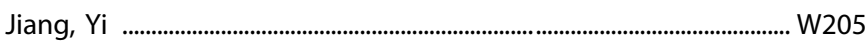

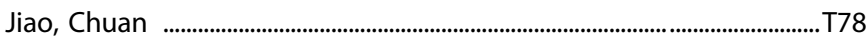

Jimenez, Amy M. ..........................................................................................M165

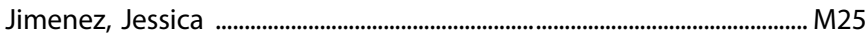

Jimmy, Jagan .................................................................................................. T30

Jo, Booil ...................................................................................................................155

Jo, Jonathon .................................................................................................. W146

Joanlanne, Andrea .............................................................................................. W90

Joëls, Marian ....................................................................................................... M79

Johansen, Annette ............................................................................................... T177

John, Hoekman ........................................................................................................ W192

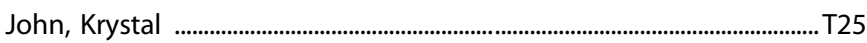

Johnson, Amy ................................................................................................. T279

Johnson, Bernard .................................................................................................. W216

Johnson, Emma ..................................................................................................... T240

Johnson, Jessica . ..............................................................................................W52

Johnson, Katherine ........................................................................................ M84

Johnson, Matthew ................................................................39.5, M226, W102

Johnson, Megan A ..................................................................................... M256

Johnson, William _...............................................................................................M272

Jokinen, Jussi ................................................................................................ M71

Jonak, Carrie ................................................................................................. M40

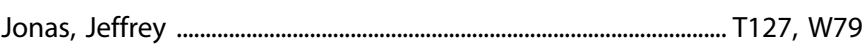

Jones, Carrie ............................................................................................ M258, W159

Jones, Delaney .......................................................................................................... T106

Jones, Jason ........................................................................................................ T93

Jones, Matt .............................................................................................................. M56

Jones, Rebecca .................................................................................................... T62

Jones, Sara ............................................................................................................... T277

Jones, Tom ............................................................................................................. T2

Jones-Brando, Lorraine .................................................................................. M90

Jordan, Chloe ............................................................................ T50, W271

Joshi, Shantanu .............................................................................................. T29

Joshi, Somala .................................................................................................. W33

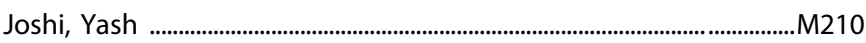

Jouroukhin, Yan ....................................................................................... T275

Jovanovic, Tanja ..................................................13.2, T7, T8, T98, W23, W53

Ju, Anes ............................................................................................................. T86

Juarez, Barbara .................................................................................... 23.1, 23.4, T89

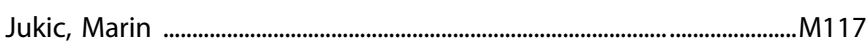

Jung, Yon Woo ............................................................................................... T2

Justice, Amy ........................................................................................................ W233

Jutla, Amandeep ..........................................................................................M192

Kable, Joseph ............................................................................................... W202

Kaczkurkin, Antonia .............................................................................. W178

Kadriu, Bashkim ................................................................................ T161, T164

Kahn, Rene ......................................................................................................... W176

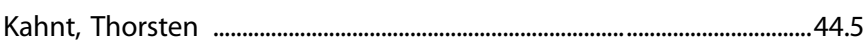

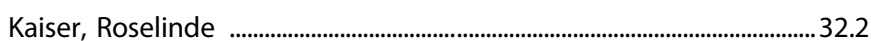

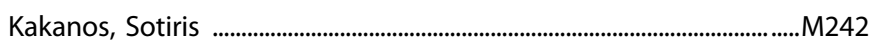

Kakar, Rishi ................................................................................................................ M72

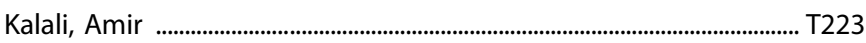

Kalin, Marc ........................................................................................................... T155

Kalin, Ned ............................................................. 49.3, M96, T74, T79, T103

Kalivas, Peter ............................................................ 9, 17.3, T54, W225, W245

Kallestrup, Peter ..................................................................................................W2

Kallioniemi, Elisa ............................................................................................. T156

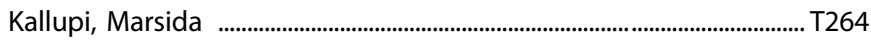

Kamiya, Makoto ............................................................................................. T126

Kämpe, Robin ......................................................................................... T250

Kampman, Kyle ...............................................................................T248, W263

Kana, Veronika ............................................................................................. T142

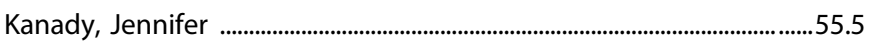

Kanba, Shigenobu .......................................................................... M109, M203

Kane, John .......................................................................................... 3, 3.4, W201

Kane, Louisa ........................................................................................................... T55

Kane, Patrick ........................................................................................................ T11

Kaneda, Yasuhiro ............................................................................................ M66, W1

Kanekar, Shami ..............................................................................................139

Kanes, Stephen ............................................... M143, M144, T127, T138, W79

Kang, Dongchon ..............................................................................................109

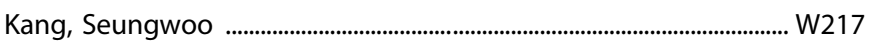

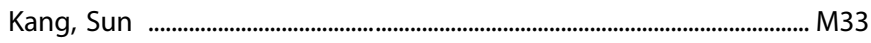

Kangas, Brian .............................................................................................. W1

Kanno, Atsuo ................................................................................................. T178

Kano, Shin-ichi .......................................................................................... T190

Kantrowitz, Joshua .............................................................................................. M164

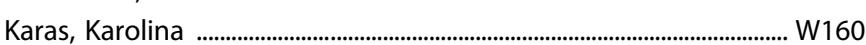

Karbalaei, Reza .................................................................................................... T234

Kardon, Randy ..................................................................................................... T181

Kareken, David .......................................................................................................M269

Karim, Helmet .................................................................................................. M30

Karkhanis, Anushree ............................................................................................ T277

Karmakar, Chandan ................................................................................................ T28

Karoly, Hollis ............................................................................................................ W243

Karp, Barbara I. ........................................................................................... T176

Karpyak, Victor ............................................................................W265, W272

Kash, Thomas ...................................................................................... 40.3, T56

Kashiwazaki, Hiromi .................................................................................. T206

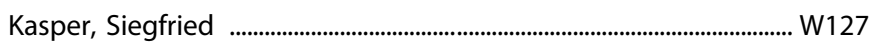

Kato, Daisuke ................................................................................................ W282

Kato, Takahiro …………………………………………………………………….....

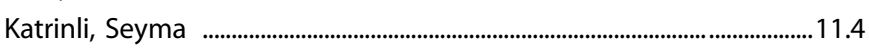

Kaundinya, Gopinath ........................................................................................... M4 M

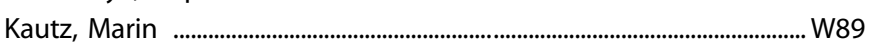




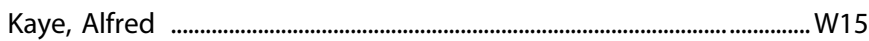

Kaye, Walter …………………………........................................................ T121

Kayser, Matthew ..................................................................................................22.1

Kayser, Reilly ..................................................................................................... T33

Kazimi, Iram ....................................................................................... T143

Keaney, Gregg …………………………………………………………..... T226

Kearley, Nicholas ............................................................................................ T23

Kearney-Ramos, Tonisha ......................................................................... T262

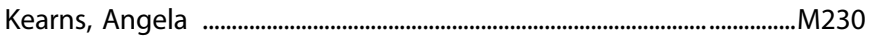

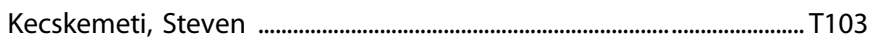

Keding, Taylor ................................................................................................T72

Keedy, Sarah ..................................................................... M112, M223, T37

Keefe, Richard ..................................................................................................W77

Keeley, Robin ............................................................................................. W246

Kegeles, Lawrence ............................................................................. M164, T219

Keighron, Jacqueline ................................................................................ W271

Kellendonk, Christoph .......................................................................T42, W69

Keller, Sune H ............................................................................................... T177

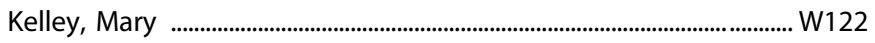

Kelliher, Julia ................................................................................................M272

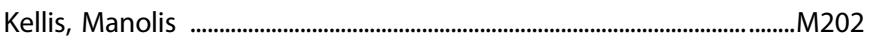

Kelly, Deanna ................................................................................................... 160

Kelly, Emily ..................................................................................................... M37

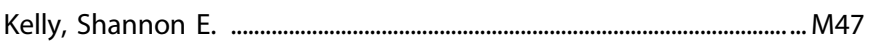

Kelly, Sinead ......................................................................W167, W170, W188

Kelmendi, Benjamin ................................................................................................. 39.6

Kenna, George .......................................................................................T51

Kennedy, Andrew ............................................................................................. M51

Kennedy, James ................................................ 47.2, M288, T35, T171, W196

Kennedy, Nicole M. ..................................................................................... T268

Kennedy, Pamela .............................................................................................M228

Kennedy, Sidney ..............................................................................................4. 45.1

Kenny, Paul .............................................................................. 56, M271, W248

Kent, Justine ............................................................................68, M69, W204

Kentner, Amanda ............................................................................................... T101

Kenwood, Margaux ..................................................................................996, T79

Keogh, Conor .......................................................................................... W145

Kepecs, Adam ......................................................................................... 24.5, M73

Kerman, Ilan ....................................................................................................... W8

Kerns, Suzanne E. ............................................................................................. W138

Kerr, Jennifer R. ................................................................................................. W131

Keshavan, Matcheri ........................M183, M207, T37, W167, W170, W188

Keuthen, Nancy ......................................................................................... W154

Khalil, Huzefa ........................................................................................................... M77

Khalsa, Sahib ....................................................................................................... T121

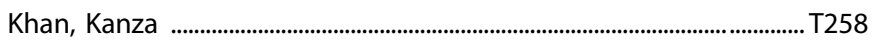

Kheirbek, Mazen ..............................................................................................M140

Khokhar, Jibran ........................................................................................ W232

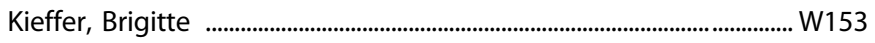

Kieslich, Karel .................................................................................................. W10

Kilian, Hannah ...................................................................... M118, W124, W155

Kilpatrick, Lisa ......................................................................................... W140

Kilts, Clinton .........................................................................................M19, W115

Kilts, Jason ................................................................................ T183, W11, W287

Kim, Angela ................................................................................................................ W6

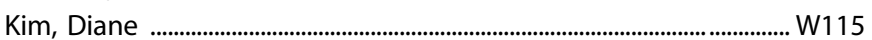

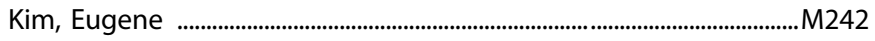

Kim, Ho-Cheol .......................................................................................................... 38.8

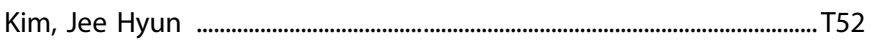

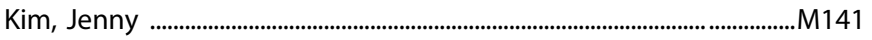

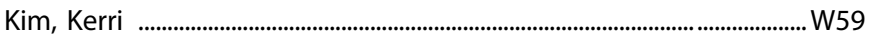

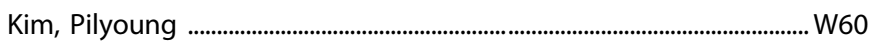

Kim, Seung-Goo ........................................................................................... W157

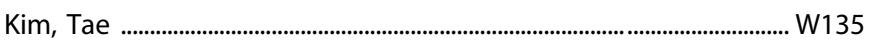

Kim, Ye Ji ........................................................................................................ T8

Kimbrough, Adam ........................................................................................M25

Kimmel, Mary ................................................................................................. T27

King, Andrea ...................................................................................... M220, T60

King, Anthony ......................................................................................... M11, T88

King, Courtney ............................................................................................. W247

King, John ..................................................................................................... T3

Kingsbury, Marcy ......................................................................................W36

Kinkead, Becky ................................................................................W92, W125

Kinney, Kaitlin ................................................................................................ T247

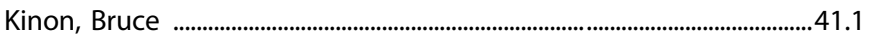

Kippenhan, J. Shane ..............................................................M283, T166, W40

Kippin, Tod ................................................................................................. T281

Kiraly, Drew .................................................................................................. W266

Kircanski, Katharina .....................................................M56, M80, M282, W13

Kirlic, Namik ...............................................................................................W24, W41

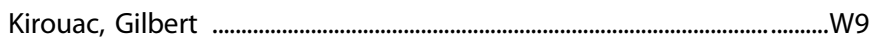

Kirpalani, Anish .............................................................................................................. M64

Kish, Steve ................................................................................................................ W62

Kisner, Alexandre .............................................................................................. M44

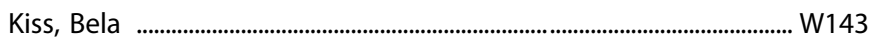

Kitamura, Takashi ..............................................................................................T39

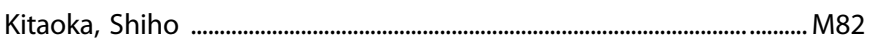

Kito, Takuya ....................................................................................................... T178

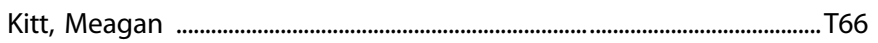

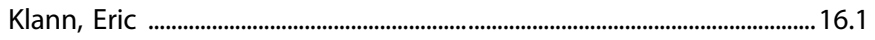

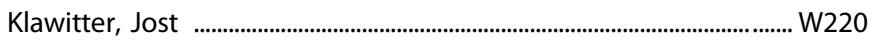

Klein, Matthew ............................................................................................... T21

Kleinman, Joel ....................................................................... M175, M176, T224

Klengel, Claudia .............................................................................................. M79

Klengel, Torsten .....................................................................................M79, T82

Klimes-Dougan, Bonnie ....................................................................................M113

Kline, Emily .............................................................................................................. W179

Klodnicki, Michael .......................................................................................... T264

Kloiber, Stefan .................................................................................................... M22

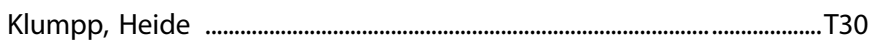

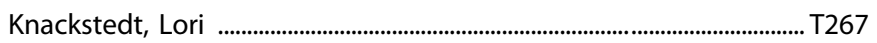

Knickmeyer, Rebecca .............................................................................226, T27

Knight, Bettina ............................................................................................... T155

Knight, Rob ....................................................................................... T231

Knowles, Emma ............................................................................... M48, T131

Knudsen, Gitte .............................................................................T177, W121

Knutson, Dan .............................................................................................. W107

Koblan, Kenneth ..........................................................................T215, W204

Kochunov, Peter ............................................................................... M128, W112

Kocsis, James ................................................................................................ W89

Koenig, Aaron ................................................................................... M143, M144

Koenigsberg, Harold .................................................................................. 32, 32.3 
Koesmahargyo, Vidya

Koethe, Dagmar

M187

Kohler, Christian

W200

Kohlnhofer, Bridget

... W78

Kohn, Jordan

Kohn, Philip

.....W5

Kohtz, Amy

M29, M283, T166, W40, W211

Kohut, Stephen

Koike, Hiroyuki

$\mathrm{T} 270$

Koizumi, Teruki

T133, W226

Kokdere, Fatih

T167

Kokkinou, Michelle

M174

Kolb, Hartmuth

...W96

Kolla, Nathan

W160

Kollins, Scott

$55,55.8, \mathrm{~T} 108$

Komanchuk, Jelena

T106

Kondo, Yuji

T201, T206

Konishi, Kyoko

.... M3

Konova, Anna

. .14 .5$

Konradsson-Geuken, Åsa

T196

Koob, George

10.3, W250, W270

Kooiker, Cassandra

Kopp, Richard ............................................................................................... W205

Korczak, Daphne ...................................................................................................... W93

Korenic, Stephanie ........................................................................................ M189

Korgaonkar, Akshata ...................................................................................... M250

Koscik, Timothy ............................................................................................. T182

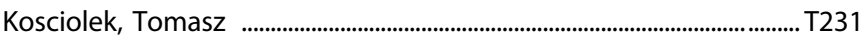

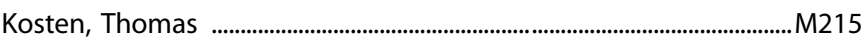

Kotz, Juliana ....................................................................................................159

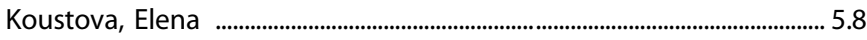

Kowalczyk, William ............................................................. M237, W254, W256

Kozauer, Susan ........................................................................................... W123

Kozbial, Piotr ................................................................................................. W100

Kozel, F. Andrew .......................................................................................... T149

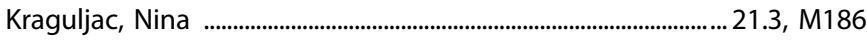

Kramer, Caroline ............................................................................................... M64

Kramer, Michael .................................................................................................. W142

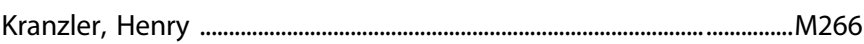

Kraus, Christoph .............................................................................................. T144

Krause, Beatrix ................................................................................................ W140

Kreek, Mary Jeanne .........................................................................................M256

Kreifeldt, Max ................................................................................................................M255

Kremen, William .............................................................................................. W108

Kretsge, Lisa ......................................................................................................125

Kreuder, Ann-Kathrin .......................................................................................... W16

Kreyenbuhl, Julie ........................................................................................... W180

Krishnan, Harish .......................................................................................... T271

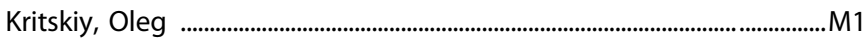

Kroes, Roger ..................................................................................................

Krogh, Kelly ..................................................................................................... M99

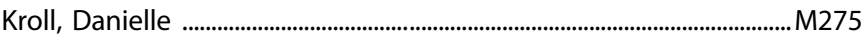

Kronman, Hope ........................................................................................ W221

Krug, Marie ............................................................................................... T113

Kruse, Jennifer ........................................................................................................ M81

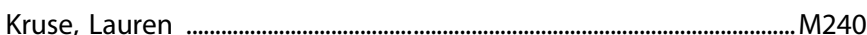

Kruyer, Anna

. W225

Krystal, Andrew

$10.5,45.3, \mathrm{~T} 108$

Krystal, John $38.2, \mathrm{~T} 125, \mathrm{~W} 10, \mathrm{~W} 233$

$\mathrm{Ku}$, Stacy

T89

Kuan, Elliot

T221

Kubera, Katharina

W165

Kubicki, Anthony

Kubicki, Marek

T200, W167, W170

Kubo, Satoshi

T178

Kudinova, Anastacia

..M287, W59

Kuflewski, Jennifer

M204

Kuian, Benji

M134

Kullander, Klas

W282

Kumar, Ambuj

. T149

Kumar, Anand

W116

Kumar Verma, Ravi ................................................................................... W181

Kummer, Sami ............................................................................................. T53

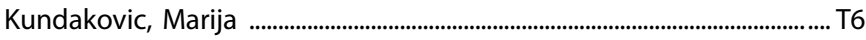

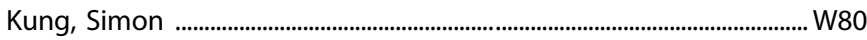

Kushan-Wells, Leila ..........................................................................................T12

Kushner, Matt .................................................................................................... W268

Kutlu, Munir ............................................................................................................ T279

Kuypers, Kim .................................................................................................................... W76

Kvarta, Mark ...................................................................................128, W112

Kwako, Laura ..................................................................................... 31.4, M246

Kwan, Alex ................................................................................................ W15

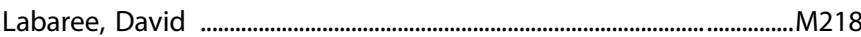

Labiste, Chase .............................................................................................44, W72

Labonté, Benoit .................................................................................................. T172

Labrecque, Simon ....................................................................................... W109

Lacefield, Clay ................................................................................................. M25

Laezza, Fernanda .................................................................................... T173

Lagishetty, Venu ........................................................................................ W140

Lago, Tiffany ......................................................................................................W17

Lähteenvuo, Markku ......................................................................................... W198

Lahti, Adrienne ...........................................................................................

Lai, Meng-Chuan ................................................................................................. T92

Lai, Ngai C. ................................................................................................................... W32

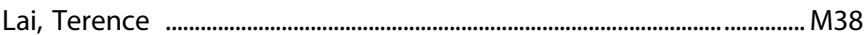

Laird, Angela .......................................................................................................... 4.3

Lajtha, Abel ..................................................................................................... T193

Lallai, Valeria ............................................................................................................M150

Lally, Níall ............................................................................................................. W133

Lam, Sherry .......................................................................................M232, W141

Lambert, Karlie ....................................................................................................M227

Lamberth, Erin ................................................................................................. T242

Lamberty, Gegory ....................................................................................... T78

Lamon-Fava, Stefania .................................................................... W92, W125

Land, Benjamin ............................................................................................M205

Landry, Ishani .....................................................................................................

Lane, Rosanne ............................................................................................. W130

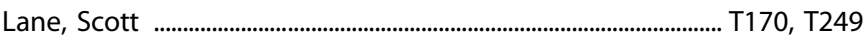

Lange, Bettina ..................................................................................... M187

Langenecker, Scott .............................................................................T30, T65

Langleben, Daniel ............................................................................................... W263 
Langlois, Jean-Baptiste ................................................................................. W280

Lanza di Scalea, Teresa .................................................................................... T130

Lanzenberger, Rupert .................................................................................. W127

Lapidus, Rachel ........................................................................................... T121

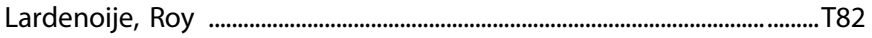

Larsen, Emmett ................................................................................................... T204

Larsen, Søren V ........................................................................................W121

LaSalle, Janine ....................................................................................................... 53.3

Lasser, Robert ..................................................................................... T127, W79

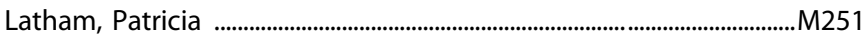

Latif, Huma ....................................................................................................... W257

Lauffer, Marisol ................................................................................................... W28

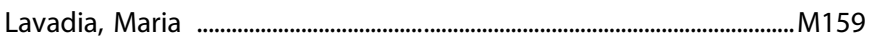

Lavretsky, Helen ......................................................................... 42, 42.5, W140

Law, Amanda ..............................................................................53, 53.2, T69

Lawrence, Andrew .......................................................................................T52

Lawrence, Steven .......................................................................................T117

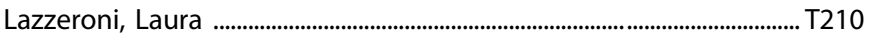

Le, Thang .............................................................................................. M36, T1 18

Le Foll, Bernard ......................................................................................M22, M237

Le Moigne, Anne ..........................................................................................161

Leach, Amy ............................................................................................. W264

Leaderbrand, Katherine ...........................................................................M247

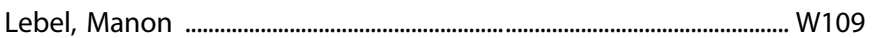

Lebowitz, Eli .......................................................................................................W13

Leboyer, Marion ..................................................................................................T28

Lecca, Daniele .......................................................................................T245, W249

LeClair, Katherine ……………………………………………………....M13, W109

Ledolter, Johannes ....................................................................................... T181

Lee, Adam .............................................................................................T37, T78

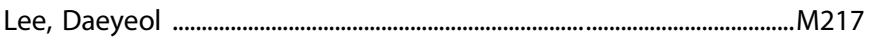

Lee, Ellen ......................................................................................38.3, T227

Lee, Francis .................................................................................................W89

Lee, Frankie ...................................................................................................... M38

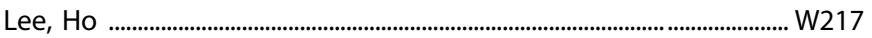

Lee, Ivy .............................................................................................................M183

Lee, Janet ........................................................................................................

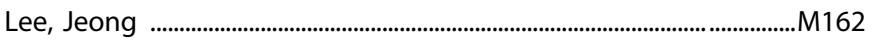

Lee, Jisoo ............................................................................................... W270

Lee, Junghee …………………………………………………..... M165, T203, T210

Lee, Jungsun ....................................................................................W167, W170

Lee, Mary .................................................................. T49, T51, T272, W252

Lee, Michelle ...................................................................................................W67

Lee, Royce ................................................................................................................. T123

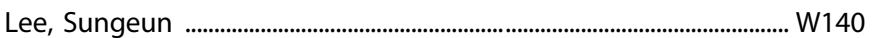

Lee, Vincent K. ..............................................................................................T99

Leet, Evan ..............................................................................................................M207

Legarreta, Margaret ..................................................................................... T114

Legget, Kristina .........................................................................................W6

Leggio, Lorenzo ................................................. M276, T49, T51, T272, W275

Lehrer, Douglas .......................................................................................... W183

Lei, Du

.T150, W113, W114

Leibenluft, Ellen .................................................. M80, M89, M282, T84, W13

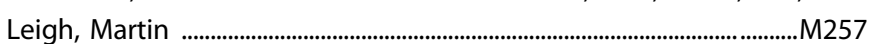

Leiser, Steven ....………………………………………………………………….... T226

Lekander, Alex .............................................................................................................M258
Lemberansky, Jamil .................................................................................. M52

Lencz, Todd .....................................................................................................W90

Lenroot, Rhoshel ......................................................................................... T216

Leonard, Michael .........................................................................................T274

León-Ortiz, Pablo ........................................................................................ W174

Leopold, David .............................................................................................. W199

Leow, Alex ................................................................................... T65, T284

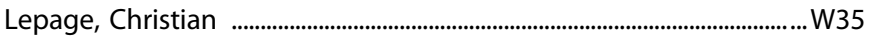

Lepow, Lauren ........................................................................................................ M59

Lerch, Jason ........................................................................ M242, T92

Lesage, Elise ................................................................................................. W267

Lesh, Tyler ..................................................................................................... W185

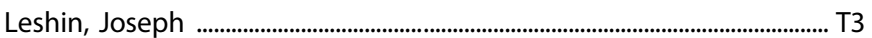

Lesiak, Atom ................................................................................M133, T265

Lesko, Emma ......................................................................................................W73

Letavic, Allyson ............................................................................................... T106

Letkiewicz, Allison ........................................................................................... T81

Letourneau, Nicole .......................................................................................... T106

Leucht, Stefan ............................................................................................... W176

Leuchter, Andrew ..............................................................................................M149

Levenson, Jessica .......................................................................................T106

Leventer, Steven ....................................................................................... M93, T147

Leventhal, Michael ........................................................................................... W282

Levi, Linda ........................................................................................................ W180

Levin, Margaret .....................................................................................................M203

Levine, Jessica ............................................................................................................ T34

Levine, Olivia ........................................................................................................... T56

Levinson, Tess _..................................................................................................... W202

Levinstein, Marjorie ...................................................................................M133

Levitt, James ………………………...............................................T200, W167

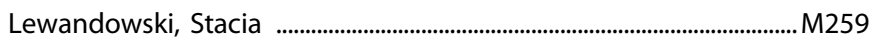

Leweke, F. Markus ..........................................................................................M187

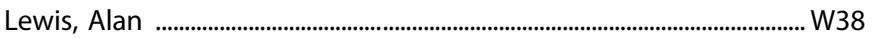

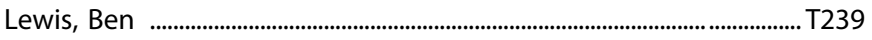

Lewis, Carolin .................................................................................................... W81

Lewis, David .......M180, M182, M204, T228, W184, W194, W195, W241

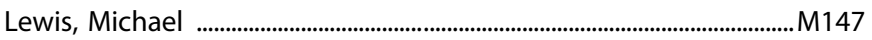

Lewis-Peacock, Jarrod .......................................................................................... M10

Leyton, Marco …………………………………………………………………….. T2

Li, Chiang-shan ........................................................................ M36, M222, T118

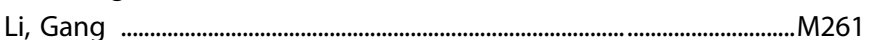

Li, Guanguan .................................................................................................... W10

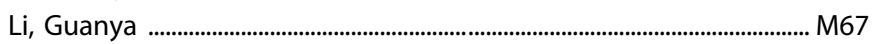

Li, Haihong ........................................................................................................... T138

Li, Hailong .........................................................................................................M156

$\mathrm{Li}, \mathrm{HaO}$..................................................................................................................... T260

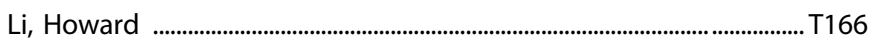

Li, Huijun ................................................................................................. W188

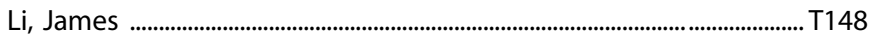

$\mathrm{Li}$, Jun-Xu ...............................................................................................W216

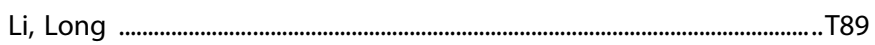

Li, Ming .............................................................................. M106, M151, T244

Li, Qingqin .................................................................................... M148, M151

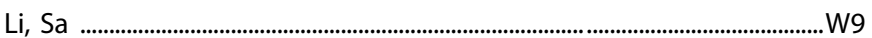

Li, Sigui ....................................................................................M144, T127, W79

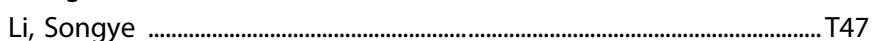


Li, Wenbin

T150, W113, W114

Li, Xiaobai

... T51

Li, Xuan

..T45

Li, Yan ..

.W65

$\mathrm{Li}$, Yulong

$\mathrm{Li}$, Zhe

W163, W164

Liang, Xiaoyu

Liberzon, Israel

Lieberman, Evan

Lieberman, Jeffrey

Liebowitz, Michael

Lieshout, Ryan Van

Lifshitz, Michael

Ligezka, Anna

Light, Gregory

Lijffijt, Marijn

Lim, Kelvin

Lim, Keunpoong

Lim, Pilar

Lima Santos, João Paulo

Lin, Amy

Lin, Eric

Lin, Hsiao-Yun

Lin, Shih-Ku .

Lindberg, Ulrich

Lindenmayer, Jean-Pierre

Lindquist, Diana

Lindsley, Craig

Ling, George

Linke, Julia

Linn, Jennifer

Linn, Kristin

Linos, Eleni

Lippard, Elizabeth

Lipska, Barbara

Lis, Cathy

Lisanby, Sarah

Liu, Cathy

Liu, Chao

Liu, Christopher

Liu, Chunyu

Liu, Fang

Liu, Heather

Liu, Jianfeng

Liu, Judy

Liu, Kalena

Liu, Keyue

Liu, Melissa

Liu, Qi

Liu, Qing-song

Liu, Rui

Liu, Shelley

Liu, Shuyan

Liu, Sihan

Liu, Siyuan

Liu, Xaioyu
Liu, Yi .. W78

Liu, Yuelu ............................................................................................................ W119

Liukasemsarn, Saran .......................................................................................197

Lizano, Paulo .................................................................................................... T37

Lizarraga, Sofia .............................................................................................. T111

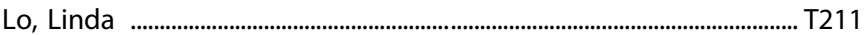

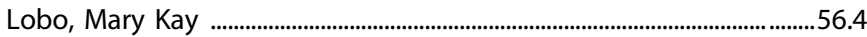

Lochner, Christine ......................................................................................... W154

Lockovich, Jeanette ........................................................... M78, T233, T283

Lodge, Daniel ...................................................................T154, W150, W228

Loebel, Antony ………………………………………....33, M68, T215, W204

Logan, Carly .................................................................................................. T267

Logan, Ryan ............................................................................................M18, W241

Logue, Mark .................................................................................................. M28

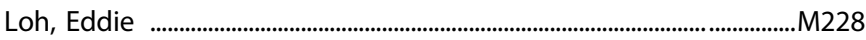

Lohoff, Falk ........................................................................T241, T251, W270

Lohse, Ines ..........................................................................................................

Lombroso, Adam ........................................................................................ T34

London, Edythe ................................................................................. T23, T247

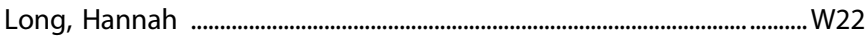

Long, Jeffrey ......................................................................................................146

Long, Victoria .............................................................................................T51

Looger, Loren ................................................................................................ W22

Lopez, Alberto ....................................................................................................... T279

Lopez, James R. ......................................................................................................165

Lopez, Sarah ............................................................................................................ T109

Lopez-Canul, Martha ..................................................................................... W153

Lopez-Gamundi, Paula ................................................................................ T170

Lopez-Guzman, Silvia ...................................................................... 31.1, 31.5

Lopez-Jaramillo, Carlos ........................................................................... W128

Lopez-Quintero, Catalina ..........................................................................M268

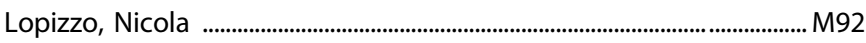

Lord, Brian ................................................................................................. W152

Lorente-Galdos, Belen .................................................................................. M34

Lorsh, Zachary .............................................................................................. T142

Loth, Meredith .............................................................................................. T28

Loudermilk, Rita ............................................................................................ W207

Loureiro, Joana ..............................................................................................T29

Lovenberg, Timothy ................................................T192, W78, W100, W152

Lovett, Jacqueline .............................................................................................. T137

Lovinger, David ...................................................................................................... W227

Lowell, Bradford .....................................................................................................W65

Lowen, Steven ........................................................................................... W191

Lowery-Gionta, Emily G. .................................................................................. W12

Lowes, Daniel ..................................................................................................125

Loweth, Jessica ..............................................................................................2225

Loydpierson, Amelia ........................................................................................... 141

Lu, Kun ........................................................................................................T2

Lu, Zhixin ................................................................................................... M48

Luber, Bruce ......................................................................................... 58.3, M9

Luciana, Monica ....................................................................................................... M57

Lucki, Irwin ................................................................................... M121, M137

Lugo-Candelas, Claudia .......................................................................... T102

Lui, Su .........................................................................................W163, W164

Luikinga, Sophia ................................................................................................T52

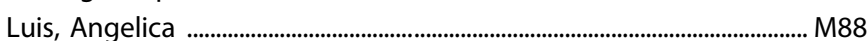


Lujan, Callan .....................................................................................................W19

Lujan, Miguel .................................................................................................... T53

Lungu, Codrin ...............................................................................................M146

Lunsford-Avery, Jessica ................................................................................ T108

Luo, Yingchun .................................................................................. M143, M144

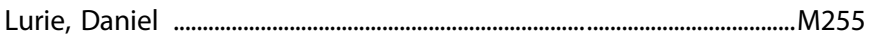

Luthringer, Remy .......................................................................................... M86

Lutz, Olivia ......................................................................................................... T37

Ly, Ronald ........................................................................................... W158

Lyall, Amanda ..............................................................................W167, W170

Lynch, Charles ............................................................................................... T62

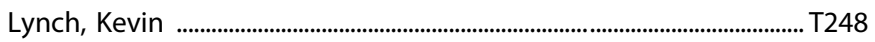

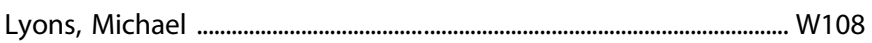

Lyu, Monica ..........................................................................................................W22

Diaz-Caneja, Covadonga M. ....................................................................... W176

Ma, Jun ..................................................................................... T26, W132

Ma, Liangsuo .................................................................................................... T24

Ma, Yizhou ........................................................................................................ T28

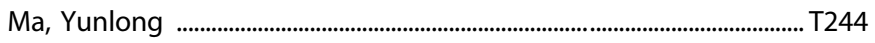

Macaluso, Matthew ...................................................................................... T148

MacDonald, Angus ............................................................. T48, T287, W268

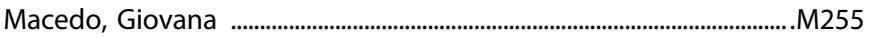

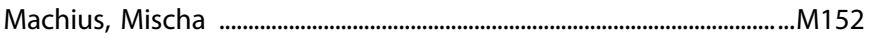

MacIntosh, Brad ..................................................................................................W93

Maciukiewicz, Malgorzata ............................................................................ W196

MacKenzie, Nicole ............................................................................... M64, T211

Mackinnon-Booth, Natalie ...................................................................... W231

MacPherson, Heather ......................................................................M287, W59

MacSweeney, Cliona ...................................................................................... M193

Madabhushi, Ram .................................................................................................... M1

Madaboosi, Shantanu ................................................................................... T5

Madangopal, Rajtarun ................................................................................... T55

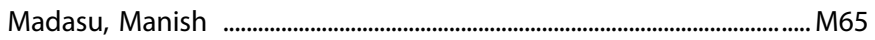

Maddock, Richard .................................................................................. W185

Madras, Bertha …………………………………………………..... T266, T270

Madrid, Annette .......................................................................................... W119

Maeda, Masashi ............................................................................................. T178

Magalong, Valerie ........................................................................................ W73

Magnotta, Vincent ....................................................................................... T18

Maguire, David ................................................................................................. W24

Mahendar, Shivaani .............................................................................................. W20

Maher, Brady ....................................................................................18.4, M51

Maher, Michael ........................................................................................... W152

Mahjani, Behrang …………………………....................................................W21

Mahjani, Christina .........................................................................................W21

Mahon, Pamela ................................................................................................M110

Maibach, Hilda ......................................................................................................M146

Maier, Steve ..........................................................................................................T66

Maione, Sabatino ...................................................................................... W153

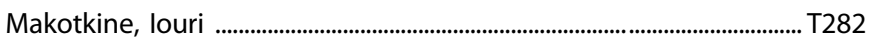

Makriyannis, Alexandros ...........................................................................W31

Maksymetz, James .................................................................................. T132

Malacon, Karen ................................................................................................W36

Malaker, Pias ........................................................................................................... W27

Maldonado, Yadira ......................................................................................... T231

Malekiani, Sam ...................................................................................................... T22
Malgaroli, Matteo M15

Malhotra, Anil 21, M168, T197, W90, W167, W169, W170

Maliackel, Lydia ................................................................................................ T93

Malievskaia, Ekaterina ................................................................................. W11

Malik, Saima ................................................................................................... M22

Malinow, Roberto ........................................................................................ T2

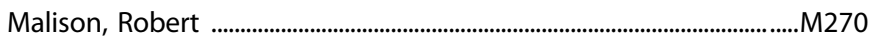

Malvaez, Melissa .................................................................................... 44.1, 44.2

Manbeck, Adrienne ......................................................................................W17

Mandelkern, Mark ................................................................................M149, T247

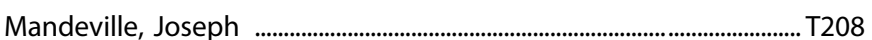

Manduca, Joshua ..........................................................................................W82

Manett, Andrew J. ....................................................................................... W138

Mangieri, Regina .......................................................................................... T259

Mangurian, Christina .................................................................................. T74

Manji, Husseini .............................................................................................. W130

Mann, Claire ...................................................................................................W31

Mann, J. John ....................................................M52, T116, T153, W97, W131

Mann, Karl .................................................................................................... W272

Mannens, Geert .................................................................................................. W117

Mannes, Andrew .............................................................................................M141

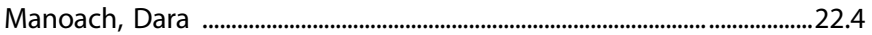

Manoochehri, Masood .................................................................................... W156

Mantsch, John ..................................................................................................... T58

Manuweera, Thushini ...................................................................................... T256

Manza, Peter ............................................................................................... M67, M275

Mao, Tianyi ........................................................................................................ 1.3

Marakhovskaya, Aleksandra .................................................................... W209

Marangoni, Ciro ......................................................................................................

Marchette, Renata ............................................................................................ W250

Marchioni, Charlotte ...........................................................................................

Marcinkiewcz, Catherine ......................................................................... T258

Marder, Stephen ........................................................................... T202, T205

Marecek, Radek ................................................................................................. M74

Mareckova, Klara ......................................................................................... M74

Marella, Julie ............................................................................................... T126

Marenco, Stefano ......................................................................................W52

Margolis, Amy …………………………………………………………...... T107

Margulies, Daniel ................................................................................................W84

Maric, Dragan ......................................................................................................M141

Marin, Marie-France ............................................................................................. M50

Marino, Patricia ...........................................................................................M130

Markovic, Tamara ......................................................................................... T115

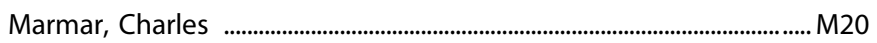

Maron-Katz, Adi ........................................................................................... M20

Marples, Brian .....................................................................................................W7

Marrocco, Jordan ............................................................................................ M77

Marsh, Nina .................................................................................................W16

Marsh, Rachel ........................................................................ M154, T107, T187

Marshe, Victoria ............................................................................................ W196

Martell, Christopher ......................................................................................... W24

Martin, Alex ...................................................................................................T62

Martin, Bill .................................................................................................... W119

Martin, Elizabeth .....................................................................................................W67

Martin, Glenn ....................................................................................................... T74

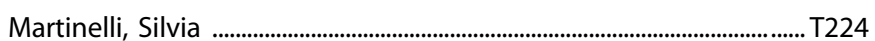




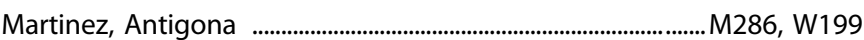

Martinez, Pedro .................................................................................................. T166

Martinowich, Keri ...........................................................................................199

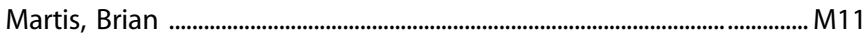

Martz, Meghan …………………………………………………......W235, W236

Marusak, Hilary .......................................................................................M31, T4

Marx, Chris

54.1, T183, W11, W287

Mary, Cosimano

... W102

Mash, Deborah C ......................................................................................... T2 T2

Mason, Natasha L ......................................................................................W76

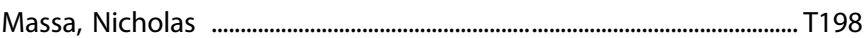

Massaly, Nicolas ............................................................................................... T115

Masson, Maxime .................................................................................................. T86

Mates, Sharon ..W123, W201, W203

Mathalon, Daniel

$\mathrm{T} 214, \mathrm{~T} 218$

Mathe, Aleksander .......................................................................... T142, W89

Mathew, Sanjay J. .......................................................................................... W285

Mathias, Samuel R. .......................................................................................... M48

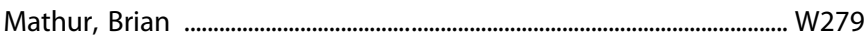

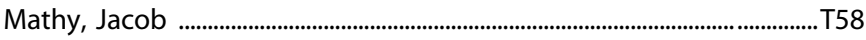

Matias, Isabel ........................................................................................... T262

Matosin, Natalie ........................................................................................... T224

Matson, Liana M. ........................................................................................... W12

Matsui, Mie .................................................................................................. W162

Matsumoto, Mitsuyuki .............................................M176, T178, T201, T206

Matsushita, Karin ............................................................................................... W162

Mattay, Venkata S ................................................................................................ M283

Matthew, Horchar ............................................................................................. T141

Matthews, Gillian ............................................................................................ W144

Matthews, Robert .............................................................................................. W159

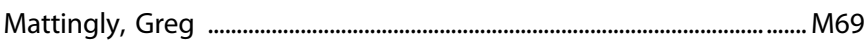

Maturana, Alejandro ...................................................................................... T41

Matuskey, David ...........................................................M241, T25, W10, W219

Matyas, Csaba ............................................................................................. W2

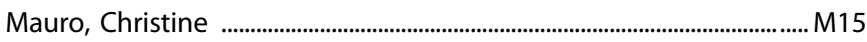

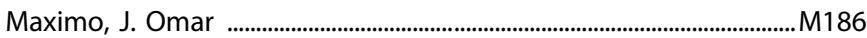

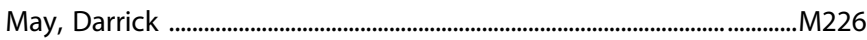

May, Matthew D. .............................................................................................. W12

Mayberg, Helen .......................................................................................35.5, T184

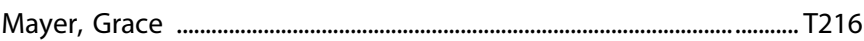

Mayfield, Brent .......................................................................................................... M51

Mayfield, Dayne ..................................................................................T246, W229

Maynard, Kristen ..............................................................................................M199

Mayo, Leah ..................................................................................................... T250

Mayol, Rocio ...................................................................................................... T41

Mazei-Robison, Michelle .......................................................................... W224

Mazid, Sanoara .....................................................................................................M256

Mazingue, Cecile M. ................................................................................W138

Mazzelli, Monica .................................................................................M92, W172

Mazzu, Maria ...................................................................................................... M97

McAdams, Carrie ................................................................................................ T1

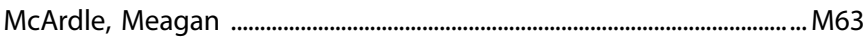

McCaffrey, Alicia .............................................................................W103, W135

McCall, Jordan .......................................................................................... M65, M129

McCall, W. Vaughn ............................................................................................. W138

McCarley, Robert .................................................................................... M203, W18

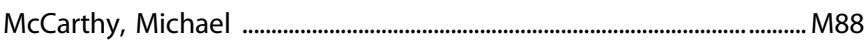

McClay, Mason ....................................................................................................... M10

McCleery, Amanda ..................................................................................... T203

McClernon, F. Joseph ............................................................................. W260

McClintick, Megan ....................................................................................... T247

McClintock, Shawn ................................................................................. T156

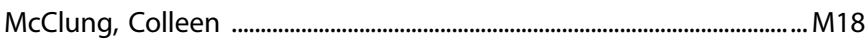

McCord, Mikayla ............................................................................................. T111

McCrindle, Brian ..............................................................................................W93

McCullough, Kenneth .................................................................M8, M79, T80

McCullumsmith, Robert .............................................................W147, W177

McCutcheon, Robert ...................................................................................... M190

McDermott, Tim ...................................................................................................W24

McDonald, Laura ...............................................................................................M210

McDonald, William ........................................................................................T74

McDougle, Christopher ............................................................................... T10

McElligott, Zoe ..................................................................................................W20

McElroy, Susan ..............................................................................................W137

McEwen, Bruce .............................................................M77, M256, T142, W89

McFaddin, John ...............................................................................................

McFague, Nina .......................................................................................... T268

McFarland, Minoo ........................................................................................... M275

McFerren, Amber ................................................................................................ T286

McGarry, Andrew ................................................................................................M146

McGee, Sean ...........................................................................................................M120

McGlade, Erin .............................................................................................................. T114

McGowan, Josephine C .................................................................................. T137

McGuire, Philip ................................................................................................... W176

Mclnnis, Melvin .................................................................................................M134

Mclntosh, Andrew ................................................................................... T131

Mclntyre, Roger ................................................................................................. M99

Mclyntyre, William ......................................................................................... T2 11

McKee, Sherry ...................................................................................................... T47

McKellar, Heather ............................................................................................ 15.3

McKenzie, Barbara .........................................................................................T149

McKinney, Brandon ..........................................................................................204

McKissick, Sara ............................................................................................. T106

McLaughlin, Katie ..................................................................................T72, T94

McLaughlin, Nicole ..........................................................................................M158

Mcloughlin, Declan ......................................................................................... W118

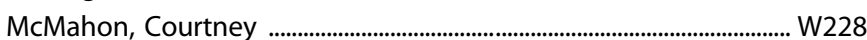

McMahon, Francis ........................................................................................ T131

McMurray, Matthew .................................................................................... W69

McNamara, Robert ..................................... T150, T197, W113, W114, W284

McNutt, Markey ....................................................................................................... M63

McPherson, Katherine ...................................................................................... M275

McPherson, Trevor ....................................................................................... T286

McQuail, Joseph .......................................................................................... T2

McReynolds, Jayme ................................................................................... T58

Meabon, James ...................................................................................... T18

Meagher, Chelsea ...........................................................................................M224

Meaney, Michael .............................................................................. M77, T142

Mechawar, Naguib ........................................................................................ W109

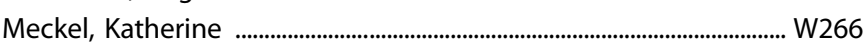

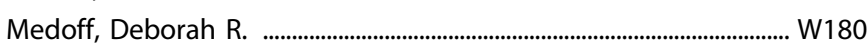

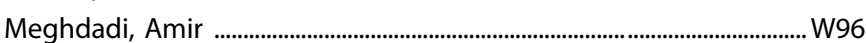




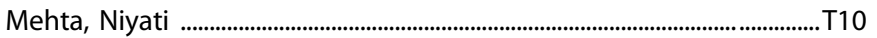

Meldrum, Sheila ..................................................................................................M119

Melhem, Nadine ............................................................................................ T116

Mellado, Elena Alvarez .................................................................................M209

Meller, Jarek .............................................................................................. W177

Mellick, William ............................................................................................T159

Meltzer, Herbert

46, 46.3, M208, W196

Meltzer-Brody, Samantha

M97, T27, T138

Menard, Caroline

T142, W109

Mennerick, Steven ..................................................................................................54.3

Mennigen, Eva ........................................................................................................T12

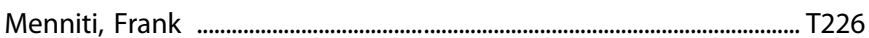

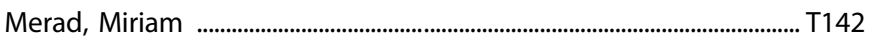

Mericle, Susan ......................................................................................................... T1 19

Merikangas, Kathleen ................................................................................ M7, M33

Mermon, Diana ................................................................................................M207

Merranko, John ...............................................................................T135, W103

Merrill, Brian .................................................................................................... W183

Meruelo, Alejandro ........................................................................................ M41

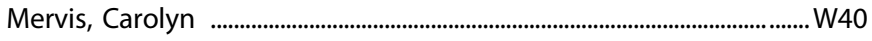

Messing, Robert ....................................................................................... T246

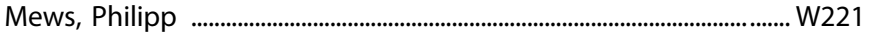

Meyer, Dora ................................................................... M118, W124, W155

Meyer, Jeffrey .....................................................................................................W62

Meyer, Thomas ................................................................................................... W237

Meyer, Urs ...........................................................................................................W34

Meyer-Lindenberg, Andreas ....................................................................... W182

Meyers, Barnett ..................................................................................M130, W178

Mian, Yeunus ………………………………………………………………..... W10

Michael, Andrew .............................................................................................. M58

Michaelides, Mike .................................................M232, W141, W246, W271

Michelini, Giorgia .................................................................................................... 4.5

Michelotti, Enrique ........................................................................................ 5.1

Michopoulos, Vasiliki ........................................................................... T8, W23

Miczek, Klaus .............................................................................................. T274

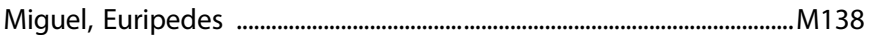

Mihara, Takuma ………………………………………………....... T178, T195

Mihov, Yoan .......................................................................................................... W83

Mikulich-Gilbertson, Susan ......................................................................... W60

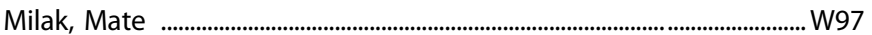

Milak, Matthew ................................................................................................ T153

Milani, Ana Carolina ................................................................... T83, T102, W8

Milenkovic, Marija .............................................................................................. T270

Milic, Milos ...............................................................................................................W5

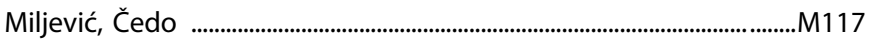

Miller, Andrew ........................................................................ M135, T198, 101

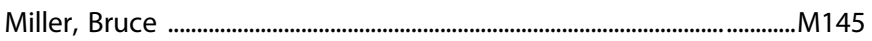

Miller, Courtney ......................................................................................... 5, 17.2

Miller, Jacob .............................................................................................. T182

Miller, Jeffrey ....................................................................................... T116, T153

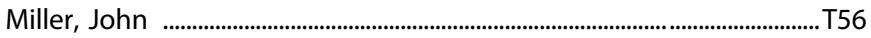

Miller, Keith ..............................................................................................................T90

Miller, Lauren ..........................................................................................................M207

Miller, Nicholas ................................................................................................M209

Millett, Caitlin ............................................................................................... M95, T162

Millman, Zachary ……………………………………………………………..... W179

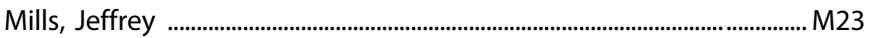

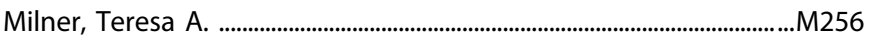

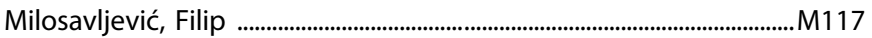

Milovanovic, Mike ........................................................................................M225

Mimura, Masaru ………………………………….... T133, W162, W168, W226

Min, Eli ............................................................................................................... T153

Min, Sarah ............................................................................................................ T49

Minhajuddin, Abu ................................................................................................ M89

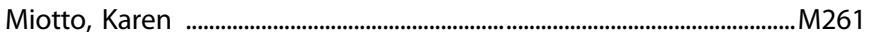

Miranda, Dayton ..............................................................................................168

Miri, Pardis ......................................................................................... T77

Mischoulon, David ............................................................................ W92, W125

Mishra, Himanshu ................................................................................................... M88

Misra, Anurag .........................................................................................................M152

Missig, Galen .............................................................................. 19.3, T10, T80

Mitchell, Danae ...............................................................................................T69

Mitchell, Jennifer ............................................................................... 59.2, W120

Mitelman, Serge ..............................................................................................W183

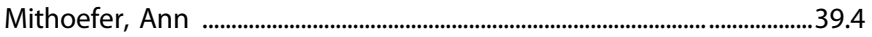

Mithoefer, Michael .............................................................................................39.3

Mitra, Robi ..................................................................................................................... 1.2

Mittendorfer-Rutz, Ellenor .......................................................................... W198

Miyamoto, Yoshiaki ........................................................................................... W186

Miyazaki, Takahiro ………………………………………………………...... W162

Miyazaki, Tomoyuki ...................................................................T133, W226

Mizrahi, Romina .....................................................T36, T237, W160, W175

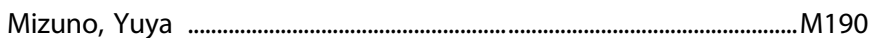

Mkrtchian, Anahit ...........................................................................W105, W133

Moaddel, Ruin .................................................................................................. T137

Modarresi, Farzaneh ...............................................................................................W7

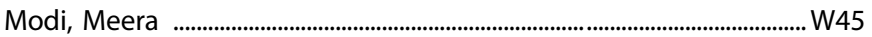

Moeller, F. Gerard .......................................................................................... T249

Moerke, Megan ........................................................................................... T273

Mohammadi, Shahin ....................................................................... M8, M202

Mohan, Amrita ..............................................................................................M147

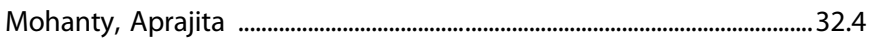

Mohazzab-Hosseinian, Sahra ...................................................................... T164

Mohr, David ...................................................................................................... W134

Mokler, Emery .................................................................................................... T10

Molden, Espen ......................................................................................M117

Molina, Juan ....................................................................................... M210, T40

Moline, Margaret ................................................................................................M211

Mollon, Josephine ……………………………………………........ M48, M167

Momenan, Reza .....................................................................2263, T241, T256

Moncada-Habib, Tomás ................................................................................. W174

Mondal, Prithu .................................................................................................. W107

Mondimore, Francis M. ................................................................................ T148

Mondragón-Maya, Alejandra ................................................................. W174

Mong, Jessica .............................................................................................. W213

Monge, Zachary ........................................................................................... W263

Monk, Catherine .........................................................................43.2, T104, W49

Monk, Christopher ................................................................................................ 13.4

Monlezun, Stephanie ....................................................................................... T262

Monroy-Jamarillo, Nancy .............................................................................. W98

Monsour, Molly ......................................................................................................... T85

Montel, Rachel A. ........................................................................................ T2 
Montgomery, Sarah ...................................................................................... T89

Monti, Elisa ................................................................................................................. T87

Montoro, Rodrigo ........................................................................................ T273

Montoya, Amanda ......................................................................................M253

Moody, Jason ................................................................................................ T103

Mooney, Larissa ...................................................................................... T247

Moon-Kim, Sam ........................................................................................ W241

Moore, Eileen ........................................................................................................ T95

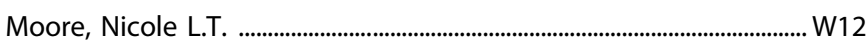

Moore, Raeanne .................................................................................................. T65

Moore, Tyler ................................................................................................ M27, T93

Mora-Durán, Ricardo ....................................................................................... W174

Morath, Brooke .................................................................................. M120, T90

Morel, Carole .................................................................................................... T89

Morell, Christopher ............................................................................................ W169

Morency, Daphnée ....................................................................................... T172

Morey, Rajendra ..................................................................... M58, T85, W287

Morgan, Judith .............................................................................................. T1 10

Morgan, Peter ................................................................................................. W208

Morgan, Robert ..............................................................................................W137

Morilak, David ............................................................................................... W228

Morishita, Hirofumi ........................................................................T109, W282

Moritz, Amy .................................................................................................. W181

Moron-Concepcion, Jose ................................................... M250, M265, T115

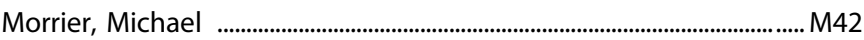

Morris, Benjamin .................................................................................................... W243

Morris, Evan ............................................................................................................. M217

Morris, James ................................................................................................... T251

Morris, Natalie ............................................................................................... T155

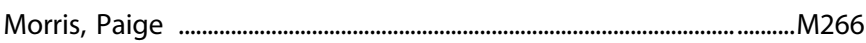

Morrison, Kathleen ................................................................................................. T96

Morrow, A. Leslie ..........................................................................54, 54.2, T31

Morrow, Jonathan ........................................................................................M284

Mortensen, Ole ...............................................................................................259

Morton, Lucas ........................................................................................................... W189

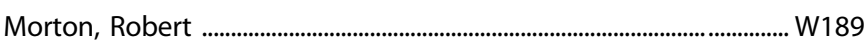

Mosconi, Matthew .............................................................................................. M47

Moseman, Scott ............................................................................................. T121

Moskal, Joseph ..................................................................................... M101, M247

Moskowitz, Bailey ........................................................................................... T246

Mossman, Sarah .................................................................................................... M23

Mould, Richard .................................................................................................M193

Mounts, Erin ................................................................................................... T65

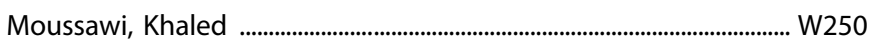

Mucci, Armida ....................................................................................................... W176

Mueller, Bryon ....................................................................................................M113

Mueller, Daniel ............................................................................20.1, 47.4, W196

Mueller, Juliane K. .......................................................................................187

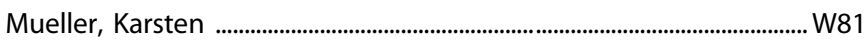

Mueller, Lauren ................................................................................................. T43

Muench, Christine .......................................................................................... T241

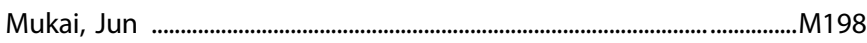

Mukherjee, Jogesh .................................................................................... W183

Mukhopadhyay, Partha ............................................................................... W270

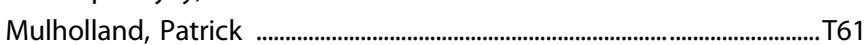

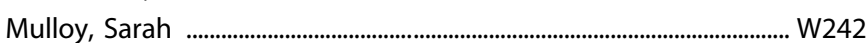

Mulsant, Benoit ................................................................................. M130, W178

Mun, Jiyoung .................................................................................................. W66

Murata, Stephen ........................................................................................... W139

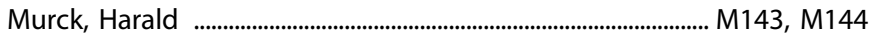

Murgas, Matej ............................................................................................. W127

Murphy, Caitlin .................................................................................. T201, T206

Murphy, Michael ......................................................................................... W139

Murphy, Nicholas ......................................................................................... W285

Murray, Betsy .................................................................................................................... 44.4

Murray, Conor ..............................................................................................M225

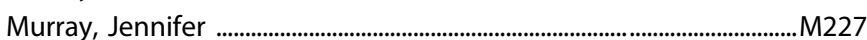

Murray, Robin ....................................................................................................M167

Murrough, James ..........................................................................22.5, W88, W89

Murthy, Venkatesha ..................................................................................... W74

Mustoe, Audrey .................................................................................................... T242

Muzi, Mark ....................................................................................................... T185

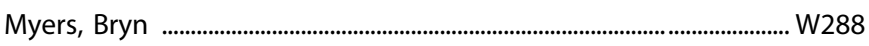

Myin-Germeys, Inez ..................................................................................... M167

Myoraku, Alison ............................................................................................... W89

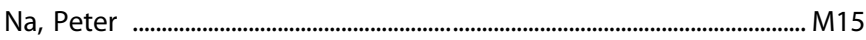

Nabeel, Nabulsi ................................................................................................ T276

Nabel, Elisa ............................................................................................................. T109

Nabulsi, Nabeel ........................................................... M218, M241, T25, W219

Nachnani, Rahul ............................................................................................W32

Nadav, Tali ............................................................................................................. T25

Nagai, Nobuhiro ....................................................................T133, W168, W226

Naganawa, Mika ................................................................................... T25, T276

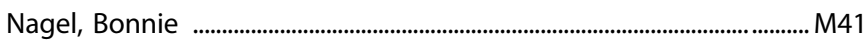

Nahas, Ziad H. ........................................................................................... W138

Naim-Aricha, Reut ...................................................................................... M96

Nair, Sunila .................................................................................................. M133

Najafzadeh, Soheila .................................................................................. T47

Nakajima, Shinichiro .........................................................T133, W162, W226

Nakajima, Waki ..............................................................................T133, W226

Nakamura, Alyson ............................................................................................ M63

Nakamura, Kazuhiko ..................................................................................... W48

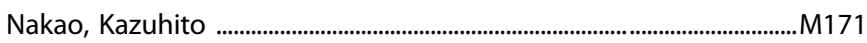

Nakazawa, Kazutoshi ......................................................................................... M171

Narasimhan, Meera ........................................................................................... T207

Narasimhan, Srinivasan ....................................................................................... W57

Narayan, Vaibhav ..........................................................................................M151

Narr, Katherine .......................................................................34.5, M81, T29

Nasca, Carla ................................................................................. 2.3, T142, W89

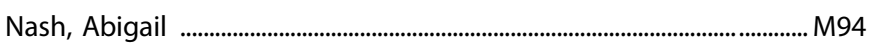

Nash, Tiffany ....................................................................................................W40

Nasrallah, Henry ....................................................................................... W189

Nassim, Marouane ................................................................................... T142

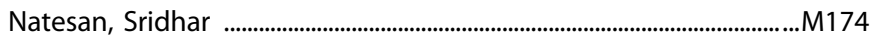

Nathan, Pradeep ............................................................................. M181, M193

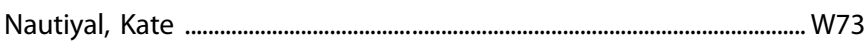

Nawaz, Uzma .................................................................................................183

Naylor, Jennifer ............................................................. 54.4, T183, W11, W287

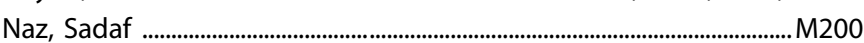

Nazeri, Arash .................................................................................................M191, W35

Needham, Stormy ................................................................................................ T139 
Neigh, Gretchen

M6, W23

Nellis, Julie

T181

Nelson, Andrew

T165

Nelson, Brady

M105

Nelson, Charles

W45

Nelson, Eric

M186

Nelson, Peter

.T65

Nemati, Samaneh

M107

Nemeroff, Charles

T74, T130

Nennig, Sadie

Nestler, Eric

M116

Neufeld, Nicholas

M228, T52, T142, W221, W231

Neumaier, John

Neumann, Peter

...M133, T265

W231

New, Antonia

. .47 .7$

Newbern, Jason

W218

Newcomer, John

M161, M196

Newman, Amy

... T50

Newman-Tancredi, Adrian .......................................................................... W280

Newport, Donald ............................................................................................ T155

Neylan, Thomas ………………………………………..........................W19

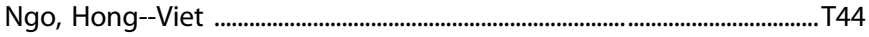

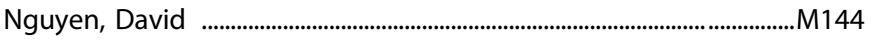

Nguyen, Jenny …………………………………………………………….... T101

Nguyen, Nghi D. ............................................................................................... T173

Nguyen, Nhien ........................................................................................... T120

Nguyen, Tanya ……………………….......................................................... T231

Nguyen, Thi Mai Loan ........................................................................... T137

Nickerson, Lisa ........................................................................................M49, T7

Nickl-Jockschat, Thomas ......................................................................... W182

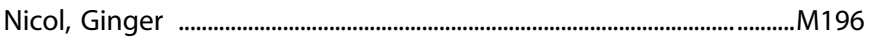

Nie, Xiang ................................................................................................. M82

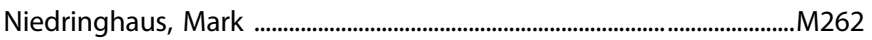

Nielsen, Mette ........................................................................................................ W176

Niendam, Tara .......................................................................................... T113

Nierenberg, Andrew ............................................................ M91, W92, W125

Nierenberg, Jay .......................................................................................... W178

Nieto-Castanon, Alfonso .......................................................................... W188

Nieto-Estevez, Vanesa ................................................................................... W228

Nikolova, Yuliya ..................................................................................................... M74

Nilson, Ashley .........................................................................................................M201

Nimarko, Akua ...................................................................................127, W94

Nimgaonkar, Vishwajit .....................................................................M207, T131

Nimitvilai, Sudarat .......................................................................................... T61

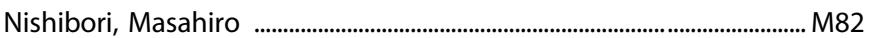

Nishino, Izumi ............................................................................................... T126

Nishitani, Shota ................................................................................................. M4

Nitta, Atsumi ...................................................................................................W186

Nixon, Sara ......................................................................................... T239

Niznikiewicz, Margaret ................................................................................ W18

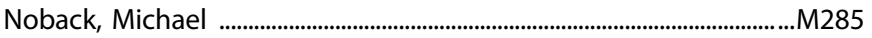

Noda, Yoshihiro ........................................................................................ W162

Nopoulos, Peg ............................................................................................... T182

Norbury, Agnes ............................................................................................ T204

Nordeman, Anton ........................................................................................... W251

Norman, Kevin

..T109, W282

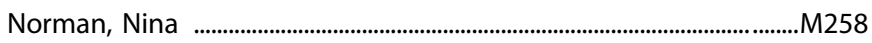

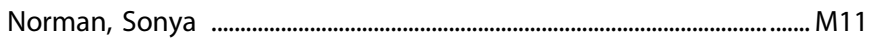

Northrup, Jessie B. .........................................................................................T99

Nouel, Dominique ............................................................................................ T53

Nourduri, Sirisha .......................................................................................... T112

Nrusimha, Ananya ......................................................................................... 127

Nuechterlein, Keith H. .................................................................. T203, T210

Nugent, Allison C. .............................................................................................. W133

Nugent, Bridget ............................................................................................. T96

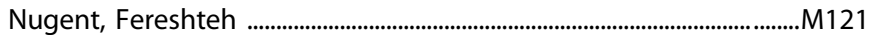

Nunez, Leyna ................................................................................................. T1

Nunez, Nicolas .......................................................................................... W137

Núñez Estevez, Karen ............................................................................T101

Nungaray, John .................................................................................................M210

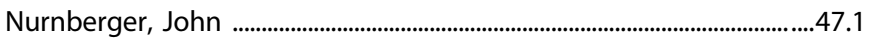

Nusslock, Robin .................................................................................. 29.1, 29.3

Nye, Jonathon .................................................................................................. W66

O'Doherty, John P. ...........................................................................................T13

O'Malley, John .......................................................................................................W9

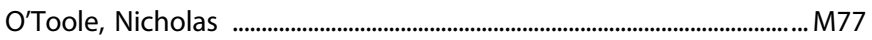

Oathes, Desmond ..................................................................T76, W22, W283

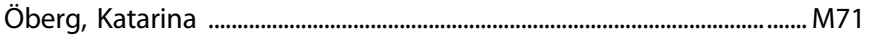

Oberlander, Tim .................................................................................................M28

O'Brien, Amanda ........................................................................................................ W45

O'Brien, Charles ................................................................................................... W263

O'Donnell, James .............................................................................M26, W151

O'Donnell, Lauren ........................................................................................... T200

O'Donnell, Patricio .................................................................................................W74

O'Donovan, Bernadette ................................................................................ T254

O'Donovan, Sinead ........................................................................................... W147

Ofotokun, lgho .............................................................................................W23

Ogden, R. Todd ………………………………………………...... T116, T153

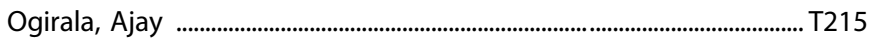

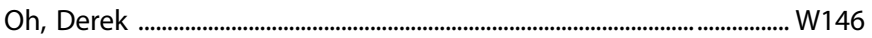

Oketokoun, Zainab ........................................................................................M271

Olaniran, Adedayo ……………………………………………………..............T45

Olfson, Emily ........................................................................................... T34

Olfson, Mark ............................................................................................................... M91

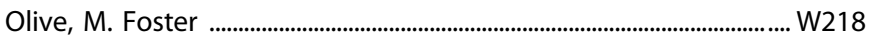

Oliver, Jason ....................................................................................................... W260

Olivo, Leah .....................................................................................................W9

Olmstead, Cory ....................................................................................................M281

Olmstead, Richard ................................................................................................. M81

Olsavsky, Aviva .............................................................................................W60

Olson, Elizabeth .................................................................................................... M24

O'Malley, Stephanie ......................................................................... M241, W272

Ombach, Hendrick .......................................................................................M139

Omberg, Larsson .............................................................................................. T65

Ong, Mei Sing ................................................................................................ W50

Ongur, Dost ........................................................................................ M197, T221

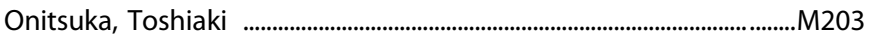

Oot, Emily ................................................................................................... M49

Oquendo, Maria ……………………….........................30.2, M52, T153, W97

O'Rawe, Jonathan ..............................................................................................M102

Ordoñes Sanchez, Evelyn ................................................................................ T234 
Oribe, Naoya

M203

Orsini, Caitlin

W70, W72, W255

Ortinski, Pavel

$\mathrm{M} 235, \mathrm{~T} 254$

Ortiz, Maria

W250

Oshinsky, Michael

41.4

Osmundsen, Allison

Ospina, Luz T204

Oswell, Corinna

M265

Otero, Marcela T185

Othy, Shiva ... T22

Otte, Kaitlyn

Ouchi, Atushi

Ousley, Opal

Overby, Paula

... M42

Owen, Randall

W218

Owens, Michael

M93, T147

Oxenkrug, Gregory

. T155

Ozburn, Angela

... M166

Ozenne, Brice

M267, T259

. T177

Pace, Thaddeus

.... T8

Pacher, Pal

W270

Padilla-Coreano, Nancy

Pagliaccio, David

W144

Pagulayan, Kati M154, T84, T107, T187

Palagani, Ajay

.T261

Palekar, Nikhil

.....W4

Palmer, Abraham

. T240

Palmer, James

M100, T75

Paloczi, Janos

W270

Pan, Lisa

.T116

Pan, Pedro

...M138

Panda, Roshni

M64, T194

Pandey, Ghanshyam

W85

Pandey, Subhash C.

T271, W276

Panigrahy, Ashok

Panlilio, Leigh ...T99

Panuwet, Parinya

W254, W256

Papalino, Marco

. T198

W209

Papendorp, Carin ................................................................................... T111

Parameshwaran, Dhanya ........................................................................... W104

Pardis, Parnian ..................................................................................................... T194

Pardo, Jose ...................................................................................................... M200

Parellada, Mara ......................................................................................................W42

Parent, Sophie .................................................................................................... T238

Parente, Camila ................................................................................................ T102

Parikh, Sagar V. ........................................................................................... T148

Parikh, Vinay ................................................................................................ W149

Parise, Lyonna .......................................................................... M13, T157, W61

Park, Lawrence ...................................................................................................... T161

Parke, Gillian ...................................................................................... T183, W11

Parker, Ian .................................................................................................. T22

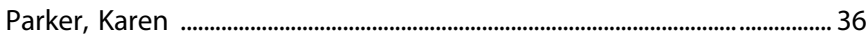

Parker, Krystal ....................................................................................................M194

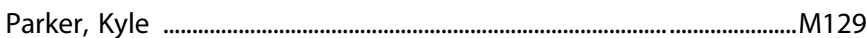

Parolin Jackowski, Andrea

T83, W8

Parsey, Ramin

W97, W115

Partilla, John W230

Parvaz, Muhammad

4.1, M59, W277

Pasternak, Ofer

W167, W170, W188

Patel, Gaurav

M286, W199

Patel, Marguerite M215

Patel, Mehul .................................................................................................................... M99

Patel, Nirali .................................................................................................................. M207

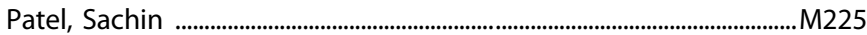

Patel, Trusharth ....................................................................................... 135

Patel, Zena ............................................................................................................. M63

Paterson, Clare ................................................................................................... T69

Patil, Shruti ............................................................................................................. T246

Patino, L. Rodrigo ..............................................................T150, W113, W114

Patrick, Finan .............................................................................................. W102

Pattabiraman, Kartik ..................................................................................... M34

Patten, Theresa .......................................................................................... T278

Patterson, Beth ................................................................................................ T18

Patterson, Jenna ....................................................................................................W37

Patterson, Thomas ......................................................................................... T207

Paul, Eleanor J. ...................................................................................................174

Paul, Elisabeth ................................................................................................. T250

Paul, Steven ................................................................................................................54.

Pauli, Wolfgang M. ............................................................................................T13

Pauls, David ..................................................................................................157

Paulus, Martin ......................................14, 38.4, M16, T121, W24, W27, W41

Pavlović, Zorana ..............................................................................................M117

Payne, Madeleine ................................................................................................ W105

Pazdrak, Konrad ....................................................................................................M238

Pearce, Bradley D ............................................................................................ T198

Pearlson, Godfrey .......................................................M48, M224, T37, W170

Pearson, Emma ...................................................................................... T256

Pechnick, Robert ........................................................................................ W146

Pecina, Marta .......................................................................................................... 7.2

Pedapati, Ernest ........................................................................................... M40, W54

Pelaez, Mari Carmen ........................................................................................... T172

Pemberton, Darrel ............................................................................... M100, T75

Pena, Catherine .................................................................................M228, T142

Peng, Kathy ........................................................................................................ T76

Penzes, Peter .......................................................................................... M195

Peralta, Juan ....................................................................................................... T131

Perera, Hassan .......................................................................................................... W25 W

Perez, Stephanie ............................................................................................. W150

Perez Algorta, Guillermo ............................................................................ T134

Pérez-Edgar, Koraly ...........................................................................................W13

Perez-Rodriguez, M. Mercedes .............................................. M95, T87, T204

Pergola, Giulio ............................................................................................. W209

Perhamus, Gretchen ........................................................................................ M80

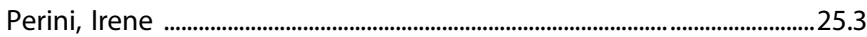

Perkel, Charles ............................................................................................................ W31

Perreault, Melissa ...............................................................................................W82

Perry, Christina ................................................................................................. T52

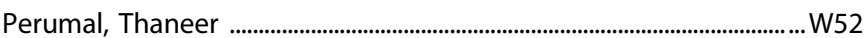

Pešić, Vesna .................................................................................................M117

Peskind, Elaine ………………………………………………………....... T185, W25

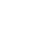


Peters, Bart ............................................................................................................ T197

Peters, Craig ...………………………………………………….......M31, T4

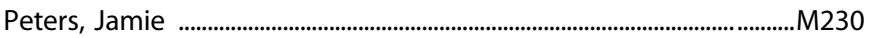

Peters, Mette ........................................................................................................W52

Petersen, Nicole ................................................................................................T23

Peterson, Abigail ......................................................................................... M32

Peterson, Bradley ......................................................................................... T104

Peterson, Drew ......................................................................................... T234

Peterson, Zeru .................................................................................................. W182

Petrie, Eric ................................................................................................. T185

Petrosino, Nicholas ......................................................................................T70

Pettit, Jeremy ...............................................................................................W13

Peyton, Lee ......................................................................................................... W217

Pfau, Madeline .................................................................................................... T157

Pfefferbaum, Adolf .............................................................................. T95, T257

Phan, BaDoi .................................................................................................... M51

Phan, K. Luan .................................................................M11, M269, T30, T284

Philip, Noah ...........................................................M21, M115, M158, T64, T70

Philippe, Julie ............................................................................................. T16

Philipsen, Alexandra ........................................................................................W16

Phillips, Karran ...............................................................................W254, W256

Phillips, Mary ............................. M78, M158, T99, T233, T283, W103, W135

Phillips, Paul .....................................................................................................240

Phillips, Rachel ..................................................................................................... M58

Phillips, Sean .............................................................................................................. T149

Philpot, Benjamin .................................................................................................... M51

Piacentino, Daria ........................................................................... T51, T272

Piazza, Piervi ............................................................................................................. T262

Pichler, Verena ............................................................................................... W127

Pietrzak, Robert ....................................................................................... W10, W219

Pignatelli, Marco ........................................................................... M232, W141

Pihl, Robert O. ............................................................................................. T238

Pine, Daniel ............................................ 49.2, M80, M282, T3, T84, T94, W13

Pini, Giorgio ................................................................................................. W145

Pinkhasov, Tzvia ...................................................................................................... M73

Pinna, Graziano .............................................................................................W276

Pisanu, Augusta ..............................................................................T245, W249

Piscitello, Andrea ..........................................................................................T65

Piser, Timothy ................................................................................................. T22

Pittman, Brian ...........................................................................................................M270

Pitts, Elizabeth ................................................................................................. W264

Pizinger, Theresa ........................................................................................... W214

Pizzagalli, Diego ........................................................................ 7.4, T168, W110

Placzek, Michael ............................................................................................ T269

Pleasure, Samuel .................................................................................................. W207

Pleil, Kristen ........................................................................................................T56

Pletnikov, Mikhail ..................................................................................... 28, T275

Plitman, Eric ............................................................................................... W162

Pochon, Jean Baptiste .................................................................... T23, T247

Pocivavsek, Ana ............................................................................................. W213

Pohl, Kilian ......................................................................................................... T95

Poland, Russell .........................................................................................W50

Polis, Ilham .............................................................................................................. T25

Pollard, Harvey ........................................................................................................ W108

Polter, Abigail ...................................................................................................... M44

Polusny, Melissa ........................................................................................................ T78

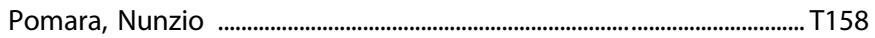

Pomeranz, Lisa .................................................................................................T14

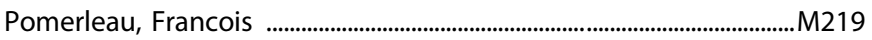

Pond, Timothy ...............................................................................................M266

Ponomarev, Igor .......................................................................................... T246

Poolman, Pieter ............................................................................................ T181

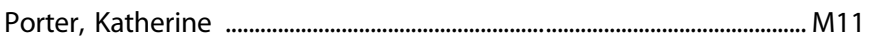

Posa, Luca ..................................................................................................... W153

Posner, Jonathan …………………………………... T83, T102, W49, W106

Post, William ...........................................................................................................M250

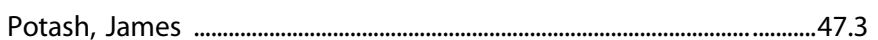

Potkin, Steven G. ................................................................................................ M163

Potter, Amelia ......................................................................................................... T106

Potter, Kenzie ................................................................................................ W234

Potter, Kevin ............................................................................................... T242

Potts, Elizabeth ............................................................................................. T186

Pouget, Jennie .................................................................................................M28

Poweleit, Ethan ................................................................................................. M23

Power, Jonathan ............................................................................................ T62

Powers, Abigail .................................................................................................... T8

Prasad, Konasale .......................................................................................... M207

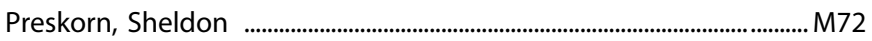

Preston, Kenzie ............................................................. 19.1, 19.5, W254, W256

Prettyman, Greer .............................................................................................. W202

Prevot, Thomas ..................................................................................................... W107

Price, David ................................................................................................................M219

Price, Destiny ..................................................................................................... M32

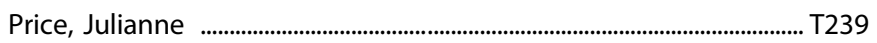

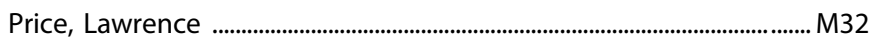

Price, Rebecca ........................................................................................ 7, 7.1, W43

Prillaman, McKenzie ..................................................................................... W246

Prisciandaro, James ......................................................................... M251, T159

Privratsky, Anthony ....................................................................................... M19

Prizgint, Lara .................................................................................................. W169

Proulx, Christophe ........................................................................................ T172

Pruitt, Christopher ............................................................................................W5

Pu, Yaoyu ...................................................................................................... W95

Puhl, Maria .......................................................................................................... T121

Pung, Meredith A. ..............................................................................................W5

Punzi, Giovanna ....................................................................................................... 12.1

Puraccio, Madeline ................................................................................................ T101

Purohit, Prerana ............................................................................................. T125

Purushothaman, Immanuel ........................................................................ T142

Pustejovsky, James ..........................................................................................M209

Qadir, Houman ................................................................................................. W279

Qian, Weilun ....................................................................................................... T132

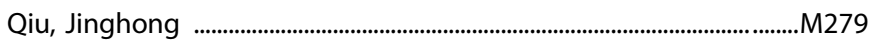

$\mathrm{Qu}$, Youge ..................................................................................................W95

Quan, Ning ....................................................................................................... T186

Quanhong, Lei ...................................................................................................... W74

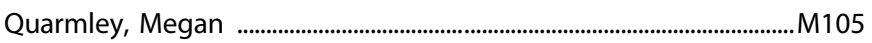

Quarto, Tiziana .................................................................................................... W209

Quehenberger, Oswald .............................................................................. T120

Quintana-Feliciano, Richard .............................................................................. T55

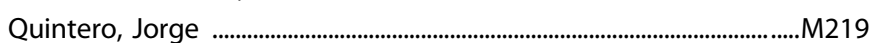


Quirk, Gregory

Quirk, Michael M143, M144, M147

Rabin, Rachel W277

Rabinak, Christine

$\mathrm{M} 31, \mathrm{~T} 4$

Rackers, Hannah

Radant, Allen D.

T210

Rader, Daniel

$\mathrm{M} 213$

Radhakrishnan, Rajiv

1276

Radhe, Ottil

T196

Radoeva, Petya M287, W59

Radulovic, Jelena M247

Ragland, J. Daniel

T113

Ragland, John

M224

Ragland, Madeline

W159

Rahman, Samir

.T222

Rainville, Jennifer

...W88

Raio, Candace

...T15

Rajagopal, Lakshmi

M208

Rajagopal, Veera

T222

Rajasekar, Thatchana

M111

Rajaskera, Therese

Rakhshan Rouhakhtar, Pamela

W179

Ralf, Regenthal ...W81

Ramakrishnan, Aarthi M228, W221

Ramakrishnan, Anantha ... 59

Ramakrishnan, Nithya

W285

Ramchandani, Vijay

$\mathrm{M} 246, \mathrm{~T} 251$

Ramirez, Steve

.... 8.4

Ramjas, Elizabeth

.T204

Ramphal, Bruce

T107

Rampino, Antonio

W209

Ramsey, Alex

14.6

Ramsey, Drew

....T74

Ramsey, Laura

Rane, Riddhi

... M23

W158

Ranganathan, Mohini

T125, T276, W208

Rangel-Hassey, Felipe W174

Rao, Manasa

Rao, Uma ... W15

Rapaport, Mark M61, M62

W92, W125

Rapkin, Andrea .....T23

Rasenick, Mark W129

Rasgon, Natalie 2, M127, T142, W3, W89

Rashidi, Mahmoud . W165

Rashidisabet, Homa ... T65

Raskin, Marissa

...T33

Raskind, Murray

T185, W25

Rasmussen, Steven

...M158

Rathi, Yogesh

W167, W170

Rathouz, Paul

M119

Rauch, Scott

M24

Rauch, Sheila

Rauh, Virginia

Ravel, Jacques

Raventós, Henriette
Ravula, Suchitra

W152

Ray, Lara

M253, M261

Rayport, Stephen ...........................................................................................T97

Razavi, Syrus ................................................................................................. T45

Raznahan, Armin ............................................................................................ M48

Rea, Jessica .....................................................................................................

Reagan, Faye ............................................................................................... T112

Reavis, Eric A. ................................................................................................165

Reckziegel, Ramiro ....................................................................................... M185

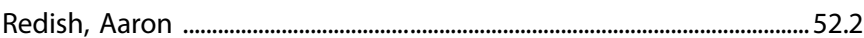

Redish, David ............................................................................................. T48

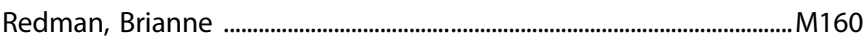

Redouté, Jérôme .......................................................................................... W280

Redwine, Laura S. ..............................................................................................W5

Reeves, Gloria ................................................................................................ W179

Refolo, Lorenzo ..................................................................................................... 5.7

Regier, Paul .................................................................................................... W263

Reichel, Carmela ...........................................................................................M230

Reichenberg, Abraham .....................................................................167, W56

Reichenberg, Avi .........................................................................................W21

Reichert, Chelsea ............................................................................................ T158

Reinhart, Robert ....................................................................................... T218

Reinwald, Jonathan ..................................................................................... W182

Reissner, Kathryn ................................................................................... 28.1, 28.4

Reljic, Tea ................................................................................................. T149

Remington, Anne ...........................................................................................M3

Remington, Gary ............................................M64, T194, T211, T225, W162

Remmersmann, Laura ..................................................................................W16

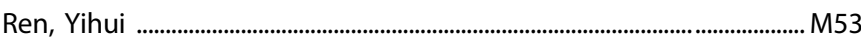

Renda, Briana ............................................................................................... M227

Rengasamy, Manivel ................................................................................... W43

Renshaw, Perry .................................................................................................139

Renström Koskela, Lotta ............................................................................ W21

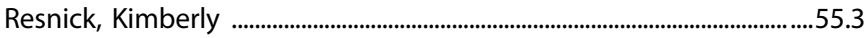

Ressler, Kerry .

M8, M79, M243, T7, T8, T80, W65

Reuter, Anne R.

M187

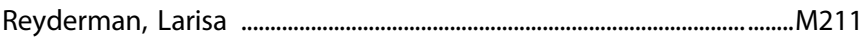

Reyes, Abner .............................................................................................. W221

Reyes-Madrigal, Francisco ........................................................................... W174

Reynolds, Charles ................................................................................................. M15

Rhee, T. Greg ............................................................................................... M91

Ricciardi, Natalie ................................................................................... W6, W7

Rice, Kenner ...................................................................................................... T66

Richard, Carson .........................................................................................................T25

Richard, Christian ..............................................................................................W96

Richard, Jocelyn ..................................................................................................M252

Richard, Keefe ................................................................................................ T207

Richardson, Charles M. .............................................................................160

Richardson, Heather .................................................................................... T112

Richardson, Kimberlei .....................................................................................W64

Richetto, Juliet .................................................................................................... W34

Richie, Christopher .......................................................................................... W141

Richter, Margaret ……………………………………………...............................T35

Riddle, Justin .................................................................................................. T286

Riedel, Marissa ............................................................................................ T103

Riedel, Philipp ...................................................................................................165 
Riesenberg, Robert ..................................................................................... T138

Rieth, Ellen ....................................................................................................... T132

Rietschel, Marcella ..................................................................................... W2

Riley, Edward ................................................................................................. T95

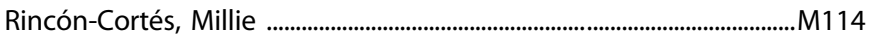

Rinker, Jennifer ....................................................................................................T61

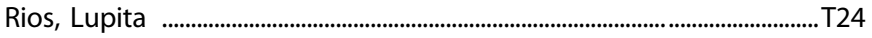

Ríos-Ortiz, Antonio .................................................................................... W174

Risbrough, Victoria ..........................................................................215, W32

Risinger, Robert ............................................................................................. M72

Rissman, Robert .............................................................................................. T22

Ritchie, Foster .......................................................................................... T111

Ritchie, James .................................................................................................. T15

Ritchie, Jobe ...................................................................................................M244

Ritchie, Max .................................................................................................... M45

Riva, Marco Andrea ....................................................................M92, W172

Rivera, Zeena ....................................................................................................M205

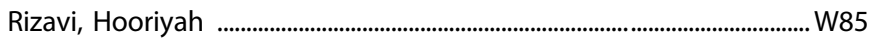

Rizk, Mina ...................................................................................................... T153

Rizor, Elizabeth ............................................................................................ T247

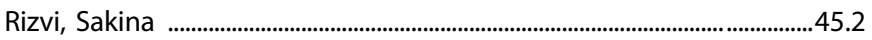

Roach, Brian ........................................................................... T214, T218

Roalf, David ..................................................................................................... M27

Roat-Shumway, Siena ....................................................................................W3

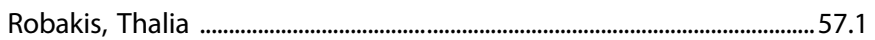

Robb, Hudson ........................................................................................... W159

Robb, William .............................................................................................M258

Robbins, James ................................................................................................ T10

Robble, Mykel ............................................................................................... W1 10

Robert, Pietrzak ...............................................................................................T25

Roberto, Marisa ......................................................................................... T25

Roberts, Amanda .................................................................. M229, T255, T259

Roberts, Bryana ......................................................................................... W260

Robinaugh, Don ............................................................................................ M15

Robinson, Andrea ...................................................................................... W234

Robinson, Delbert ..........................................................................3.1, 3.3, T197

Robison, Alfred ...............................................................................56.2, W224

Robson, Matthew ..........................................................................................T186

Roche, Daniel ............................................................................................................. T60

Rock, Rachel ................................................................................................... W144

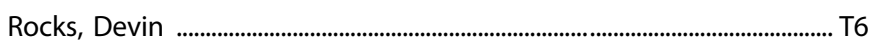

Rodd, Zachary ........................................................................................ T280

Roderick, Ryland ............................................................................................ T101

Rodger, Jennifer ............................................................................................... M85

Rodrigue, Amanda ........................................................................................ M48

Rodriguez, Carolyn ............................................................................. M155, T74

Rodriguez-Thompson, Anais ....................................................................... T63

Roffman, Joshua ...............................................................................T63, W51

Rogers, Baxter ............................................................................................ W16

Rogers, Charleanne ...................................................................................... T234

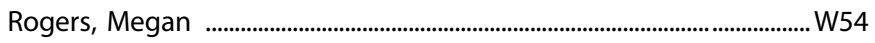

Rogowska, Jadwiga ............................................................................... T114

Rohleder, Cathrin .......................................................................................M187

Roiser, Jonathan ..............................................................................W105, W133

Rojas, Gerardo ................................................................................................... M45

Role, Lorna ................................................................................................................... W4
Rollman, Bruce ................................................................................................. W134

Román, Viktor ................................................................................................W143

Romano, Raffaella ..................................................................................... W209

Romanowicz, Magdalena ........................................................................... T90

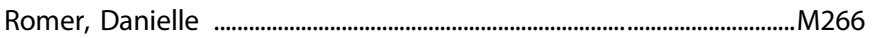

Romualdi, Patrizia ..................................................................................W153

Rondón-Ortiz, Alejandro ............................................................................ T101

Roopchansingh, Vinai ..............................................................................M263

Ropchan, Jim ...............................................................................................M241

Rosa-Neto, Pedro ......................................................................................... T1

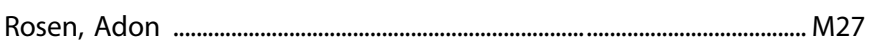

Rosen, Bruce ......................................................................................... T208, T269

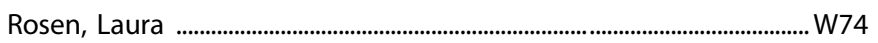

Rosenbrock, Holger ...................................................................................... W34

Rosenquist, Peter B. ..................................................................................... W138

Rosenzweig-Lipson, Sharon ............................................................................ 5.3

Rosoff, Dan ...................................................................................................... W270

Rosoklija, Gorazd B. ................................................................................. W131

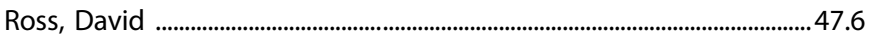

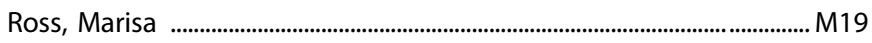

Ross, Rachel ................................................................................................W65

Ross, Thomas ………………………………………….......T252, W258, W267

Ross-Degnan, Dennis ...................................................................................... W50

Rossell, Susan ............................................................................................ T52

Rosso, Isabelle ...................................................................................................... M24

Rotenberg, Alexander .......................................................................... W45, W74

Rothbaum, Barbara ................................................................................... T7, T76

Rothmond, Debora ........................................................................... T201, T206

Rothschild, Anthony ...........................................................130, T148, W178

Rotrosen, John ................................................................................................ 31

Rotshtein, Pia ............................................................................................. W67

Roussos, Panos ................................................................................ T222, W52

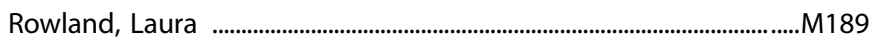

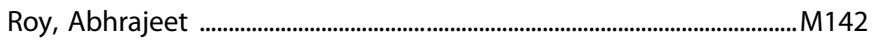

Roy, Bhaskar ............................................................................................. T145

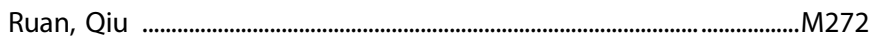

Rubin, Leah .................................................................................................. T37

Rubin-Falcone, Harry ................................................................................. T153

Rubinow, David ........................................................................ M97, T138, T16

Rubio, Javier ....................................................................................................... T55

Rubio-Lorente, Jose ............................................................................................. W90

Rucker, James ............................................................................................. W111

Rudenko, Gabrielle ....................................................................................... M152

Ruderfer, Douglas ........................................................................................ W52

Rudorfer, Matthew ............................................................................................. M130

Rudzinskas, Sarah ................................................................................................. M97

Rueda, Richard .......................................................................................................M1

Ruiz-Betancourt, Daniel ........................................................................ W199

Ruparel, Kosha ............................................................................................. M27

Rush, Augustus .......................................................................................M134

Rusjan, Pablo M. ......................................................... T36, W62, W160, W175

Russell, William .......................................................................................... W237

Russman Block, Stefanie ........................................................................ T88

Russo, Scott ............................................M13, T89, T142, T157, W109, W282

Rutherford, Saige ........................................................................................... W240

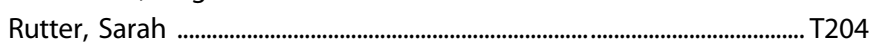


Ruzicka, Brad M202

Ryan, Arthur T.

W180

Ryan, Neal

Rychik, Natali

T242

Ryder, Benjamin

M5, T168

Rynn, Moira

Saad, Ziad

... M100, T75, W96

Sabia, Helene

T126

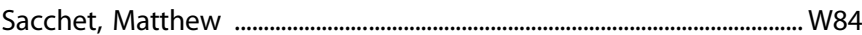

Sacher, Julia ...................................................................................................W8

Sackeim, Harold A.

W138

Sadahiro, Masato

T109, W282

Saddoris, Michael ...............................................................................................M274

Sadow, Samuel .................................................................................................. W134

Sahib, Ashish .................................................................................................T29

Sahin, Mustafa ...................................................................................................... W45

Sahlem, Gregory .......................................................................................... W138

Sahoo, Pabi .............................................................................................. T254

Saito, Manabu ................................................................................................... W48

Saito, Takeshi ................................................................................................

Salas, Ramiro ..................................................................................................

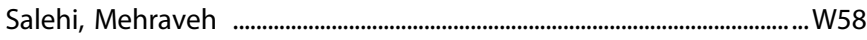

Salemink, Elske .............................................................................................W13

Salinas, Armando .............................................................................................. W227

Salmeron, Betty Jo .............................................................. T252, W258, W267

Salum, Giovanni ..................................................................................M138, W13

Samardzic, Janko ......................................................................................... W212

Sambataro, Fabio ............................................................................................. W165

Sami, Aysha ................................................................................................ T249

Sammel, Mary D. ........................................................................................... T31

Samuel, Stull .....................................................................................W254, W256

Samuels, Benjamin ........................................................................M126, T140

Sanacora, Gerard ............................................................................. M60, T125

Sanchez, Catherine ......................................................................... M155, T74

Sanchez, Deborah L. .................................................................................... T149

Sanchez Soto, Marta .....................................................................................162

Sánchez-lbarra, Jorge ................................................................................. W174

Sanchez-Pena, Juan ...................................................................................... W199

Sanchez-Roige, Sandra .................................................................................. T240

Sanchez-Soto, Marta ....................................................................... M232, W181

Sand, Michael ..........................................................................................................W34

Sandberg, Glenn ........................................................................................... W237

Sander, Christin ............................................................................................ T208

Sandhu, Thasveen .............................................................................................M155

Sandin, Sven ........................................................................................................W56

Sanguinetti Czepielewski, Letícia ..............................................................M185

Sankar, Anjali .........................................................................35.1, 35.4, M102

Sankoh, Abdul .................................................................................. T127, W79

Santiago, Jessica ..........................................................................................W24

Santosa, Hendrik ......................................................................................... T110

Saoud, Jay ........................................................................................................ M86

Saperia, Sarah ................................................................................................ T225

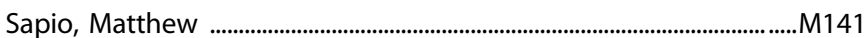

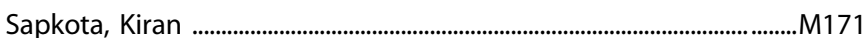

Sarabdjitsingh, Angela ........................................................................................ M79

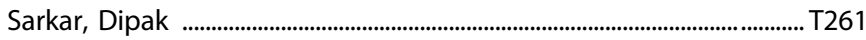

Sarpal, Deepak ...........................................................................................

Sartin-Tarm, Anneliis ..................................................................................... M19

Sartorius, Alexander ................................................................................. W182

Satlin, Andrew ....................................................................W123, W201, W203

Satterly, Kelsey ………………………………………………………….... W192

Satterthwaite, Theodore ............................................. M27, M48, W178, W202

Saunders, Benjamin ......................................................................................... M248

Savage, Cody .......................................................................................................... W270

Savard, Bradley ................................................................................................W29

Savard, Melissa ................................................................................................. T1

Savitz, Adam ......................................................................................................... M86

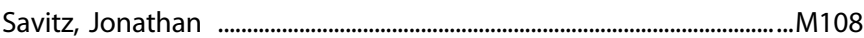

Sawada, Kyosuke ................................................................................................ W162

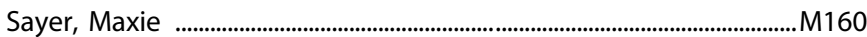

Scaini, Giselli ................................................................................................ T143

Scarborough, Joseph ...................................................................................... W34

Schachar, Russell J. ...........................................................................................T92

Schafer, Matthew ............................................................................................ T87

Schank, Jesse ................................................................................................ M116

Schaps, Rebecca ........................................................................................ T58

Scheele, Dirk .....................................................................................................W16

Scheinost, Dustin .................................................................................... T104, W58

Schettler, Pamela ................................................................................ W92, W125

Schifani, Christin ...................................................................................M191

Schiffman, Jason ................................................................................................ W179

Schiller, Crystal ........................................................................................................ M97

Schiller, Daniela ................................................................................................ T87

Schlaepfer, Thomas .......................................................... M118, W124, W155

Schlüter, Oliver ..................................................................................................... W231

Schmack, Katharina ...................................................................................... W173

Schmalbach, Therese ................................................................................... T147

Schmid, Cullen L. ......................................................................................T268

Schmidt, Anna-Maria ....................................................................................... M187

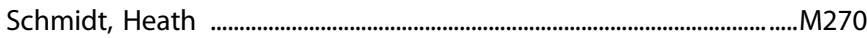

Schmidt, Mark ...................................................................................... M100, T75

Schmidt, Mathias V ........................................................................................ M79

Schmidt, Peter .................................................................................... M97, T166

Schmithorst, Vincent J. ................................................................................. T99

Schmitt, Andrea .................................................................................................. T224

Schmitt, Lauren ........................................................................... M40, M47, W54

Schneider, Elizabeth .......................................................................................... W67

Schneider, Molly ..........................................................................................................W8

Schoelerman, Jeffrey ......................................................................................... W117

Schoenbaum, Geoffrey .................................................................................... T4

Schoretsanitis, Georgios .................................................................................... W83

Schretlen, David ............................................................................................ W170

Schroder, Hans ............................................................................................... W1 10

Schroeder, Heidi ..................................................................................................... M23

Schroeder, Jennifer ..................................................................................... W254

Schroeter, Titus ..........................................................................................................W84

Schubert, Ryan .......................................................................................... W207

Schulmann, Anton .............................................................................................. W206

Schultz, Johannes ......................................................................................... W16

Schultz, Timothy ........................................................................................................151

Schurdak, Jennifer .............................................................................................. W284 
Schuster, Randi ................................................................................................... T242

Schuttenberg, Eleanor .................................................................................... M49

Schwandt, Melanie ............................................................................. M246, T251

Schwarz, Adam .............................................................................................. W182

Schwarz, Emanuel ....................................................................................W182

Schweiger, Julia ............................................................................................M196

Schwendt, Marek ............................................................................................W70

Schwieler, Lilly ................................................................................................ W197

Sciaccotta, Allegra .......................................................................................... W242

Scifo, Andrea ............................................................................................... W249

Scofield, Michael ............................................................ 28.5, M230, M264, T54

Scolaro, Kelly ................................................................................................. M52

Scolqmiero, Martina .....................................................................................W187

Scotellaro, Julia ....................................................................................................M272

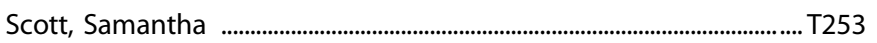

Scott, Samuel .............................................................................................. T112

Scully, Morgan ..................................................................................................... W22

Seaman, Robert ...............................................................................................M236

Sedighim, Sharona .................................................................................... T264

Seewoo, Bhedita ............................................................................................ M85

Séguin, Jean R. ........................................................................................... T238

Sehatpour, Pejman .......................................................................... M164, M286

Seidlitz, Jakob ....................................................................................................... M48

Seidman, Larry .................................................................................T210, W188

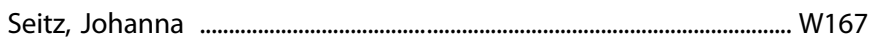

Self, David ................................................................................................................M278

Sellgren, Carl ................................................................................... M173, W197

Sellnow, Kyrie ................................................................................................... M19

Semanek, David .......................................................................................... W106

Sempio, Cristina ................................................................................................. W220

Senft Miller, Anna ............................................................................................... T68

Senthikumar, Rithika ..................................................................................... T112

Seok, Darsol ................................................................................................. W283

Sephton, Chantelle ...................................................................................... T172

Seraikas, Anna .............................................................................................. M49

Sershen, Henry .................................................................................... T158, T193

Seshadri, Saurav ............................................................................................. M83

Sestan, Nenad ............................................................................................... M34

Seth, Subhendu .......................................................................................... T230

Setlow, Barry ......................................................................M4, W70, W72, W255

Setnik, Beatrice ....................................................................................................M211

Setoyama, Daiki ............................................................................................M109

Sexton, Lacey ................................................................................................... W264

Shabel, Steven .................................................................................................... T24

Shaffer, Rebecca ................................................................................................... M40

Shafi, Reem MA ...........................................................................................T90

Shah, Relish ....................................................................................................... T97

Shahab, Saba ............................................................................................168

Shaham, Yavin ................................................................25, T55, W231, W251

Shaik, Riaz ...................................................................................................... W277

Shaista, Fnu .................................................................................................... T261

Shallcross, John ................................................................................................W70

Shampine, Larry .................................................................. T183, W11, W287

Shanahan, Megan .................................................................. M95, T162, T204

Shannon, Katherine ........................................................................................ T58

Shannon Weickert, Cynthia ........................................ T36, T201, T206, T237

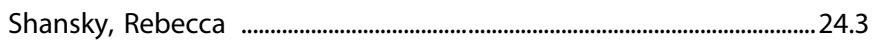

Shao, Manlin .................................................................................................... T22

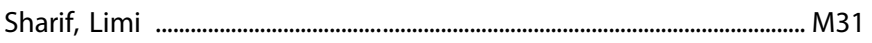

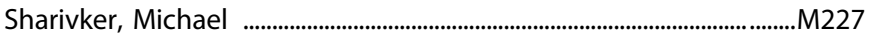

Sharma, Akul ....................................................................................... M61, M62

Sharma, Sameer ....................................................................................... T230

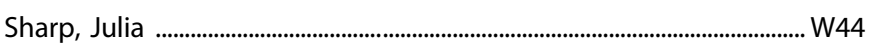

Sharpe, Melissa ................................................................................................. T44

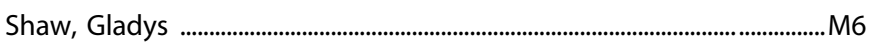

Shaw, Philip ....................................................................................................... W5

Shea, M. Tracie .............................................................................................................. T70

Shear, M. Katherine ....................................................................................... M15

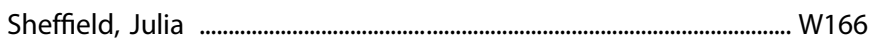

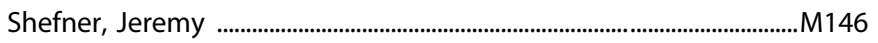

Sheikh, Sohail ............................................................................................M200

Sheline, Yvette ........................................................34, 34.2, M9, W22, W283

Shelton, Micah ................................................................................................. W241

Shelton, Richard C. ................................................................................... T148

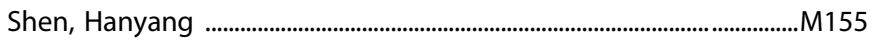

Shen, Li .......................................................................................................... M228

Shen, Pei-Hong .............................................................................................. T236

Shen, Roh-Yu ................................................................................................M257

Shenton, Martha .................................................... T200, W167, W170, W188

Sher, Leo ................................................................................................................. T282

Sheridan, Margaret ......................................................................................... T94

Sherif, Mohamed …………………………………………………………………... T125

Sheriff, Salma .......................................................................................................... T21

Sheth, Chandni ................................................................................... M139, T114

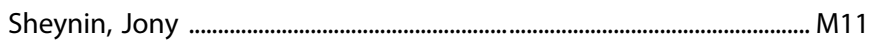

Shi, Lei ....................................................................................................................... W18

Shibata, Mikihito ...................................................................................................... M34

Shieh, Annie .................................................................................................... T271

Shih, Pei-an (Betty) ................................................................................... T120

Shin, Joo Heon ...................................................................... M51, M175, M176

Shinn, Grace ...................................................................................................M260

Shinohara, Russell ........................................................227, M48, W202, W283

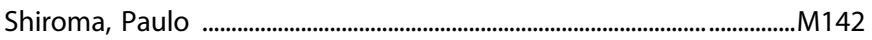

Shofer, Jane .................................................................................................. T185

Shokri Kojori, Ehsan ........................................................................ M249, M275

Shokri-Kojori, Ehsan ......................................................................................... T2 253

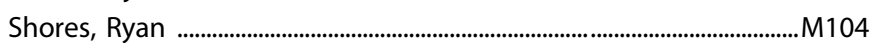

Short, E.Baron ............................................................................................. W138

Short, Jenna ................................................................................................... T109

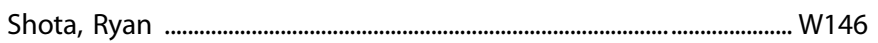

Shou, Haochang .................................................................................................. M33

Shoyombo, Ife ..............................................................................................119

Shrewsbury, Stephen ................................................................................ W192

Shtutman, Michael ...................................................................................... T111

Shukla, Rammohan ................................................................................. W177

Shuman, Tristan ........................................................................................ T263

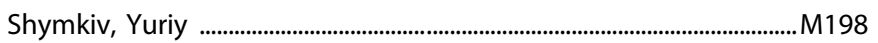

Sial, Omar .......................................................................................................W61

Sibille, Etienne .................................................................................................. W107

Sibley, David ......................................................................M162, M232, W181

Siddarth, Prabha .............................................................................................. W140

Siddiqui, Ishraq ................................................................................................. T22 


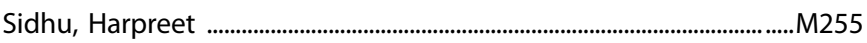

Sidoli, Simone ................................................................................................. W221

Sieberts, Solveig ............................................................................................W52

Siekmeier, Peter ............................................................................................ W191

Siemsen, Benjamin ....................................................................................... M264

Siever, Larry ................................................................................................... T210

Sil, Annesha .................................................................................................. T245

Silber, Christopher ............................................................................. T127, W79

Silva, Hernan .................................................................................................. T4

Silva, Ivaldo ...................................................................................................... T102

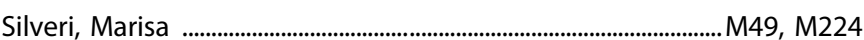

Silverman, Hannah ......................................................................................... W156

Silverman, Jeremy .......................................................................................... T210

Silverman, Wendy ..........................................................................................W13

Simkovic, Sherry ............................................................................................. M46

Simmons, Alan ............................................................................................... M16

Simmons, Sarah ................................................................................................. 121

Simmons, Trenton ........................................................................................ T243

Simmons, W. Kyle ......................................................................................... M100

Simmons, William .............................................................................................. T75

Simon, Jeremy ................................................................................................ M51

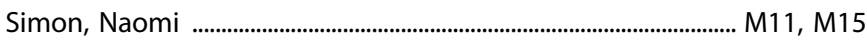

Simon, Neal .....................................................................................M146, W17

Simpson, Helen ....................................................................... T33, T187, W156

Sinacore, James ............................................................................................... M84

Singer, Annabelle ............................................................................................ T163

Singh, Aditya K ....................................................................................... T173

Singh, Jaskaran ................................................................................................ T136

Singh, Manpreet ................................................................................127, W94

Singhal, Sarthak ............................................................................................W70

Sinha, Rajita ............................................................................................... 25.5, W233

Sipahi, Eren ...................................................................................................W51

Siu, Cynthia ........................................................................................... M68, T194

Sivasubramanian, Meena .......................................................................... T270

Skelly, Mary Jane ............................................................................................T56

Skime, Michelle ............................................................................................... W2 W

Skjaervoe, Ingeborg .................................................................................. W257

Skosnik, Patrick .................................................................................T276, W208

Slade, Eric P. ....................................................................................................... W180

Slate, Sarah Rose ..................................................................................................M1 10

Slater, Rebeccah ...................................................................................................... 6.4

Slaugenhaupt, Susan ........................................................................................... 5.4

Slavich, George ................................................................................................ T68

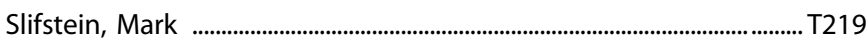

Sloand, T. Joseph ..........................................................................................M262

Sloofman, Laura ........................................................................................................ W52

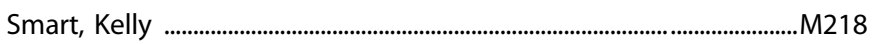

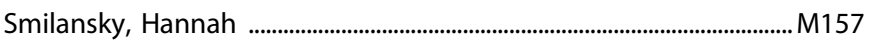

Smiley, Cora E. ............................................................................................

Smiley, John ................................................................................................ T97

Smith, Alexander ............................................................................................. W248

Smith, Alicia ........................................................................................ 11, M42

Smith, Ashley ................................................................................................. T84

Smith, Caroline ..................................................................................................W36

Smith, Daniel ................................................................................................................ W1 W

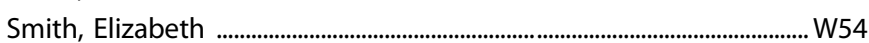

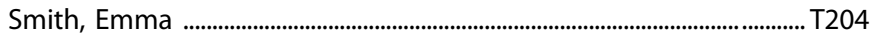

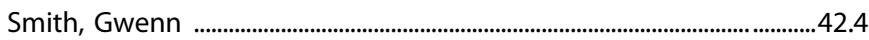

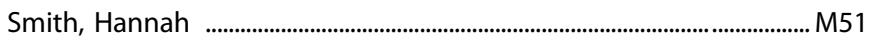

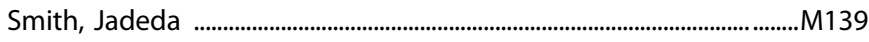

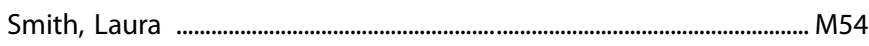

Smith, Lauren ......................................................................................... T264

Smith, Mark ......................................................................................... M174, W234

Smith, Milo .......................................................................................................... T109

Smith, Nicholas ............................................................................................ W108

Smith, Nicole ....................................................................................29, W211

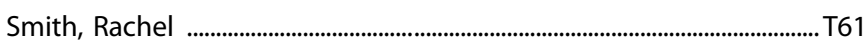

Smith, Robert .................................................................................................. T193

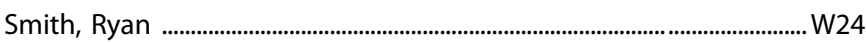

Smith, Sharon ................................................................................................... W215

Smith, Tawny L. ...................................................................................... T130

Smith, Whitney ............................................................................................ T119

Smucny, Jason .................................................................................................. T38

Sneider, Jennifer ............................................................................................. M49

Snyder, Gretchen ....................................................................................... T196

So, Jisun ....................................................................................................... W125

Soares, Jair ....................................................................................................... T143

So-Armah, Kaku .......................................................................................... W233

Sobieh, Tarek .....................................................................................................164

Soda, Takahiro ...................................................................................... 47.9, W1 18

Soder, Heather ................................................................................................... T170

Soehner, Adriane ................................................................................................ T2

Sogawa-Fujiwara, Chizuru .............................................................................. T206

Solís-Vivanco, Rodolfo .............................................................................. W174

Solli, Kristin .................................................................................................... W257

Solomon, Marjorie ........................................................................................... T113

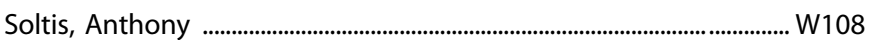

Song, David .....................................................................................................W77

Song, Mingxi ............................................................................................ T192

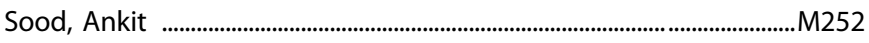

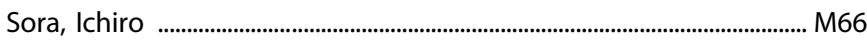

Sorcher, Leah ................................................................................................... W40

Sotiras, Aristeidis .......................................................................227, M58, W178

Soundararajan, Soundarya ......................................................................... T251

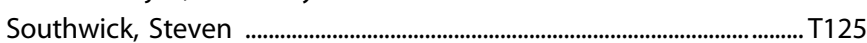

Spanier, Susanne ................................................................... M118, W124, W155

Spann, Marisa ........................................................................................... T104, W58

Specker, Sheila ......................................................................................................... T48

Spence, Jeffrey .................................................................................................M216

Spenceley, Alexander ................................................................................ W231

Spencer, Kevin .........................................................................................................M203

Spencer, Sade .......................................................................................................... W242

Spetter, Maartje ...................................................................................................W67

Spies, Marie .............................................................................................W127

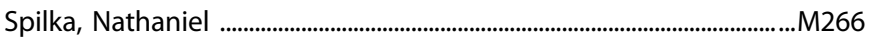

Spoon, Chad .....................................................................................................W5

Sportelli, Leonardo ....................................................................................... W209

Spriggs, Sharron ...........................................................................................W31

Sprock, Joyce ............................................................................................ T210

Spurny, Benjamin ............................................................................................ W127

Sripada, Chandra ................................................................................... T88, W240

Stange, Jonathan …………………………………………………………...T30, T65 
Stangl, Bethany

.. T251

Stankovic, Srdjan

M163

Stanley, Barbara

M52, T116

Stansfield, Katherine

... 274

Stansfield, Susan

W111

Stark, Ali

W224

Stark, Craig

M124

Stark, Shauna

M124

Starosta, Sarah

... M73

Starski, Phillip

W217

Stauffer, Chris

Stavish, Caitlin

Stefanini, Fabio

Stegmayer, Katharina

..M140

Stein, Dan

W167, W170

W154

Stein, Elliot

Stein, Murray

Steinberg, Joel

T252, W246, W258, W267

M16, T75, W27, W108

Steiner, Johann

T249

Steinglass, Joanna

M166

Stenson, Anais

27.2

Stephan, Clifford C.

T98, W53

Stephan, Klaas Enno

. T173

Stern, Sarah

Stertz, Laura

W86

Stetz, Patrick

W237

Stevens, Angie

Stevens, Jennifer

Stevens, Michael

Stewart, Evelyn

Stewart, Sujin

Stiffler, Richelle

Stirnberg, Ruediger

Stock, Janet

Stöcker, Tony

Stoddard, Joel

Stojanovski, Sonja

Stone, Craig

Stone, William

St-Onge, MP

Stott, Ryan

Stowe, Taylor

Stowe, Zachary

Strakowski, Stephen

Strauss, John

Strauss, Nicole

Strawn, Jeffrey

Stringaris, Argyris

Stringfield, Sierra

Strupp, Jacob

Styner, Martin

Su, Ping .

Su, Shelley

Su, Yujuan

Suarez, Mauricio

Suarez-Jimenez, Benjamin
Subburaju, Sivan

M202

Sublette, M. Elizabeth

M52, T116, T153

Suchankova, Petra

.T49

Sudre, Gustavo

W55

Sugar, Catherine

T210

Sugiyama, Azusa

.. M83

Suh, Jesse

W263

Suh, Junghyup

M243

Suker, Samir

Sullivan, Breanna

W83

Sullivan, Edith

.T106

Sullivan, Gregory

T95, T257

Sullivan, Patrick

.. T153

Summergrad, Paul

W118

Sun, Bomin

M166

Sun, Delin

W157

Sun, Frank ................................................................................................... T12

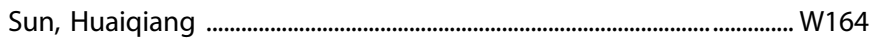

Sun, Hui .............................................................................................. T49

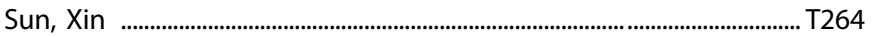

Sun, Yalin .......................................................................................... T270

Sun, Yan ............................................................................................. W108

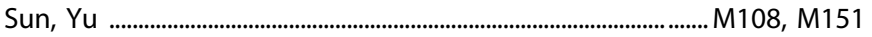

Sung, Young Hoon .........................................................................................M139

Supiot, Laura .......................................................................................................T8

Surti, Toral .......................................................................................................... W208

Sussman, Tamara ............................................................................................. T83

Sutherland, Matthew .................................................................................. W267

Suthoff, Ellison ............................................................................................ T127

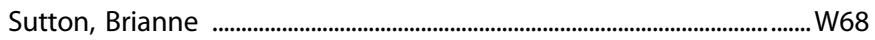

Suzuki, Masako ..................................................................................................... T6

Suzuki, Rebecca …………………………………………………………….... M15

Suzuki, Takefumi ........................................................................................ W168

Svarer, Claus ................................................................................................... T177

Svensson, Torgny H. ..................................................................................... T196

Svob Strac, Dubravka .................................................................................. W212

Swain, James ............................................................................................ W269

Swan, Julia ................................................................................................ T251

Swann, Alan C. ............................................................................................ W28

Swanson, Chelsea ........................................................................................... W287

Swardstrom, Meghan ................................................................................... W192

Swartz, Holly ............................................................................................. W134

Swearingen, Hannah ....................................................................................... T64

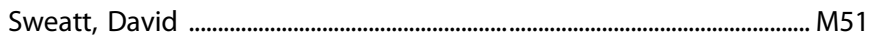

Sweeney, John M40, M47, M156, T37, W54, W163, W164, W167, W170

Sweet, Robert .............................................................. 42.2, M195, M204, T228

Sweis, Brian .....................................................................................................T48

Sweitzer, Maggie ........................................................................................ W260

Swerdlow, Neal ................................................................. M159, T40, T210

Swetlitz, Caroline ............................................................................................. T84

Swift, Robert ...................................................................................15, M276, T51

Szabo, Steven ....................................................................... T183, W11, W287

Szatmari, Peter .............................................................................................. T92

Szeszko, Philip R. …………………………………….......T197, W167, W170

Szucs-Reed, Regina .................................................................................... W263

Szuhany, Kristin ...................................................................................................... M15 
Ten-Eyck, Tyler M4, W70, W72

Tabrizi, Sarah

Taccheri, Giulia

Tadayon-Nejad, Reza

Tadross, Michael

Tagawa, Masahiro

Taipale, Heidi

Tajinda, Katsunori M83, M176, T195, T206

Takahashi, Takuya

T133, W226

Takahashi, Yoichiro

Takasu, Shotaro

W168

Takeuchi, Hiroyoshi

T194, W168

Talati, Ardesheer W106

Talbot, Austin ...T19

Talkowski, Michael

Talledo, Jo

M159, T40

Tallman, Maxwell

T150, W113, W114

Talmage, David

Tamiya, Hiroko

Tamiz, Amir

5.6, T176

Tamminga, Carol T24, T37, W109, W170, W201

Tamminga, Mila

T248

Tan, Chunfeng

Tan, Youguo

Tanase, Costin W163, W164

Tanda, Gianluigi .. W185

Tang, Sunny

W271

Tang, Yingying

W200

Tani, Hideaki .. W188

Taniguchi, Makoto T133, W168, W226

Tanum, Lars ....................................................................................................... W257

Tao, Ran ...........................................................................................M176, T224

Tapert, Susan .................................................................................................. M41

Tapia, Cynthia ............................................................................................ T173

Tarcijonas, Goda .............................................................................................172

Tardif, Christine ................................................................................................ T1

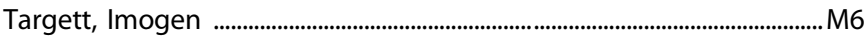

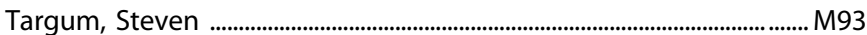

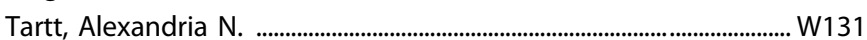

Taylor, Jane .................................................................................................................M217

Taylor, Mackenzie ...........................................................................................M221

Taylor, Margot ........................................................................................................ T92

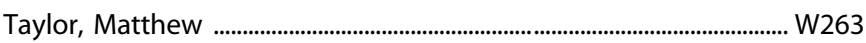

Taylor, Rachel ................................................................................................... W12

Taylor, Richard ................................................................................................... M237

Taylor, Robert ..................................................................................................W64

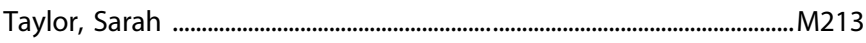

Taylor, Stephan ..................................................................................... 3.5, M102

Taylor, Valerie T. ...............................................................................................M28

Teague, Kent ................................................................................................108

Teal, Laura .................................................................................................. W159

Teboul, Eric ........................................................................................................... T42

Teichert, Tobias ..........................................................................................................M179

Teixeira, Catia ................................................................................................ T97

Terry, Garth ................................................................................................. T185

Teshigawara, Kiyoshi ................................................................................. M82

Teyler, Timothy .............................................................................................. T218

Thai, Michelle ............................................................................................. M113

Thang, LoC ..........................................................................................M65, M234

Thase, Michael ......................................................................................M103, T148

Thaxton, Courtney ............................................................................................... M51

Theriault, Rachel-Karson ...................................................................................... W82

Theyers, Athena .................................................................................................W93

Thiagarajan, Tara _............................................................................................. W104

Thibeault, Kimberly .......................................................................................... T279

Thiery, Thomas ........................................................................................................ W84

Thomas, Jason ................................................................................................ W67

Thomas, Lauren .............................................................................................. T107

Thomas, Mark ...................................................................................................... T48

Thomas, Michael .............................................................................................. T40

Thomas, Paul ................................................................................................ T284

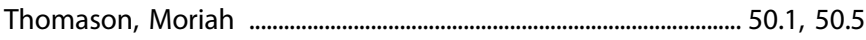

Thompson, Allison ..........................................................................................T76

Thompson, Andrew ...................................................................................... W65

Thompson, Elizabeth ................................................................................. W179

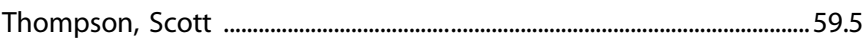

Thompson, Wesley .................................................................................. 4.4, T121

Thomson, Emma ....................................................................................................... W62

Thomson, Pippa ................................................................................................. T131

Thornell, Brenda ................................................................................................M146

Thuras, Paul ............................................................................................ M142, T78

Tiberica, Lize ...................................................................................................W2

Tice, Madelynn ...............................................................................................M224

Tieu, Lani ......................................................................................................... T264

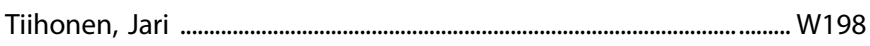

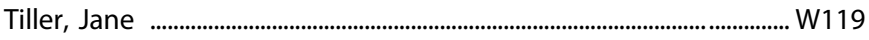

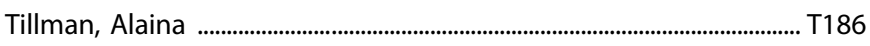

Tippani, Madhavi ..............................................................................................199

Tippler, Maria ................................................................................................ T238

Tipton, Shane ................................................................................................................ M63

Tirrell, Eric ..............................................................................................T167, W104

Titulaer, Joep .......................................................................................................... T196

Tiwari, Arun .................................................................................................T171, W196

Tobe, Russel .....................................................................................................M286

Tohen, Mauricio ...................................................................................T74, T216

Toivainen, Sanne .......................................................................................... W251

Tolliver, Bryan ................................................................................................... T159

Tomasi, Dardo ........................................................... M249, M275, T253, T285

Tomohiro, Ayaka ................................................................................................. M82

Tooley, Jessica ................................................................................................ W75

Torben, Ott .................................................................................................. W173

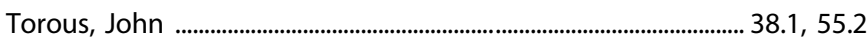

Torregrossa, Mary ............................................................................. M231, M239

Torretta, Silvia .............................................................................................. W209

Tossell, Kyoko .............................................................................................................M174

Toups, Marisa .........................................................................................M119, T130

Townsend, Edward .............................................................................................M245

Townsend, Reid ..................................................................................................... M234 


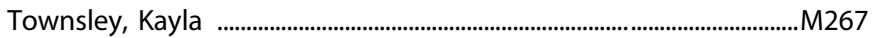

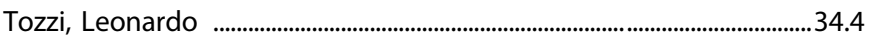

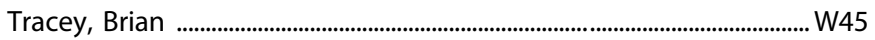

Tran, Baouyen ……………………………………………………………..... W207

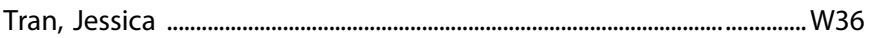

Tran, Veronica ................................................................................................ T211

Trauth, Jeanette

T106

Treadway, Michael

29, 29.5, T170

Tregellas, Jason W68

Trela, Constantine ....................................................................................T60

Tremblay, Richard E. ......................................................................... T238

Tribble, Rebekah

W60

Trivedi, Madhukar M89, M134, W99

Troiani, Vanessa 14.7

Trojnar, Eszter

W270

Trombello, Joseph ........................................................................................134

Tromp, Do ..................................................................................................... T103

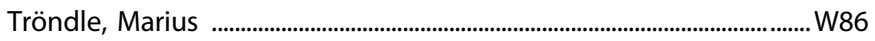

Tropea, Daniela ........................................................................................... W145

Troyer, Emily .....................................................................................................W5

Tsai, Guochuan .................................................................................................. W148

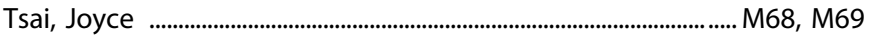

Tsai, Li-Huei ...........................................................................................................M1

Tsai, Pei-Jung ....................................................................................................... W246

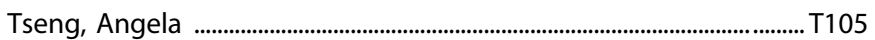

Tseng, George ................................................................................................... W241

Tseng, Wen-Yih Isaac ..................................................................................W47

Tsuang, Debby ....................................................................................................... T2 10

Tsuang, Ming ................................................................................................. T210

Tsuchiya, Kenji ..................................................................................................... W48

Tsugawa, Sakiko ......................................................................................... W162

Tsvetkov, Evgeny .........................................................................................T57

Tuck, Ellen ............................................................................................ W109

Tummula, Swetha ......................................................................................... T106

Tunstall, Brendan ............................................................................................ W250

Turecki, Gustavo ................................................................................................... W109

Turetsky, Bruce ............................................................................................. T2

Turkoz, Ibrahim ..................................................................................................... M94

Turkson, Susie ...........................................................................................................M6

Turna, Jasmine ..................................................................................................... T18

Turner, Jill ............................................................................................................ T2 254

Twiss, Jeff ............................................................................................ T254

Tye, Kay ........................................................................................................ W144

Tye, Susannah ................................................................................................M120

Tyndale, Rachel ....................................................................................... M22, T60

Tyrer, Andrea .......................................................................................................W97

Tzur, Dorit ..........................................................................................................W56

Uchida, Hiroyuki ....................................................... T133, W162, W168, W226

Uchida, Shusaku ................................................................................................... M75

Ukaigwe, Joy .........................................................................................................M176

Ukeshima, Shinya ............................................................................................ M82

Uliana, Daniela .................................................................................................... M43

Ulloa, Karen ................................................................................................ T41

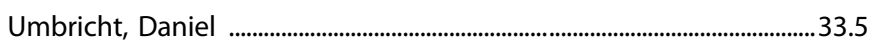

Umeuchi, Hideo ............................................................................................. T126
Umpierrez, Guillermo .......................................................................................... T8

Ungar, Lyle ...................................................................................................... W200

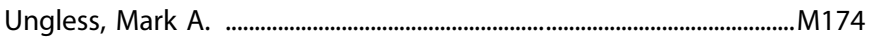

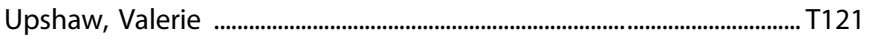

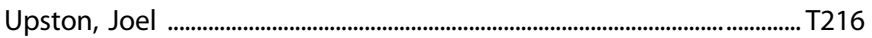

Urban, Nathan ......................................................................................................... W194

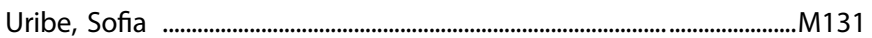

Ursano, Robert ............................................................................................. W10

Uthayathas, Subramaniam .............................................................................. W64

Uwe Foerster, Bernd …………………………………………......... T83, T102

Vaccarino, Flora ………………………………………................... 18.5, T9

Vaccarino, Viola ............................................................................................. W10

Vachez, Yvan ..........................................................................................................W75

Vadnie, Chelsea ................................................................................................... M18

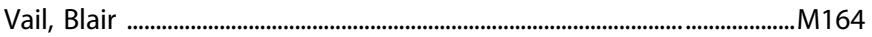

Vajdi, Ariana ......................................................................................................... T12

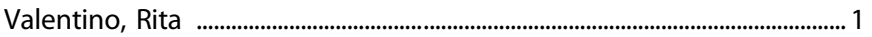

Vallee, Monique ............................................................................................. T262

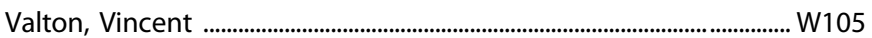

van Amelsvoort, Therese .............................................................................M181

Van Ameringen, Michael .............................................................................. T18

Van Booven, Derek ....................................................................................... W229

van der Ark, Peter .................................................................. M100, T75, W96

van der Plas, Ellen ......................................................................................... T182

Van Der Water, Judy ................................................................................ W185

van Dijk, Milenna ...................................................................................... W106

van Erp, Theo .......................................................................................... M61, M62

van Harmelen, Anne-Laura ..............................................................................35.2

Van Hedger, Kathryne ............................................................... M112, M223

Van Hove, Ilse ........................................................................................ M100, T75

Van Meter, Anna .......................................................................................... T135

Van Nueten, LuC .................................................................. M100, T75, W96

Van Os, Jim .....................................................................................................167

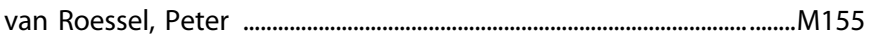

van Rooij, Sanne ...................................................................... T7, T98, W53

van 't Wout, Mascha ....................................................................... M21, T70

Van Trees, Kimberly ............................................................................................. T149

Vandekar, Simon .............................................................................................. M48

Vangel, Mark ............................................................................................................ T200

Vanover, Kimberly ........................................41.5, T196, W123, W201, W203

Vargas, Cristian ..........................................................................................W128

Varias, Andrea ...................................................................................... M155, T74

Varney, Sara ............................................................................................................... M23

Varodayan, Florence ....................................................................................... T2 255

Varol, Erdem .......................................................................................................... M27

Vasavada, Megha ................................................................................................T29

Vasdev, Neil .....................................................................................................W62

Vassileva, Jasmin ............................................................................................ 31.3

Vasudevan, Anju ....................................................................................... W193

Vawter, Marquis .................................................................................................... W206

Veenstra-VanderWeele, Jeremy ....................................................51, M192

Velarde, Nathan ............................................................................................. T264

Veldic, Marin ................................................................................................... W137

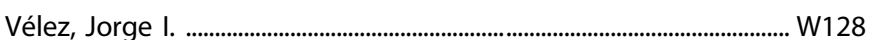

Velthorst, Eva .................................................................................................M167 


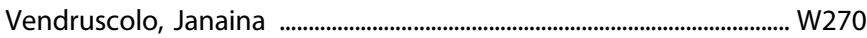

Vendruscolo, Leandro ....................................................................W250, W270

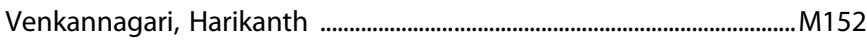

Venkatesha Murthy, Naga ........................................................................103

Venniro, Marco

$25.2, T 55, W 251$

Vento, Peter

T260

Verbeek, Marcel ............................................................................................ T158

Verghese, Cherian .......................................................................................... T202

Verite, Adrien ......................................................................................................... T86

Vermetten, Eric ........................................................................................................ 39

Vernon, Anthony ..............................................................................................M242

Veronese, Mattia ...................................................................................................M174

Verplaetse, Terril ..................................................................................................... T47

Verrico, Christopher .........................................................................................M215

Versace, Amelia ...................................................................... T99, W103, W135

Very, Madison .................................................................................................. T106

Vesel, Claudia .......................................................................................................... T65

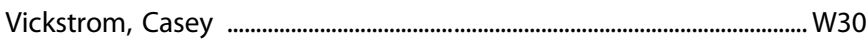

Vidal, Benjamin ............................................................................................. W280

Vieta, Eduard .......................................................................................... M99, M100

Vigod, Simone ................................................................................................. M288

Viher, Petra Verena ......................................................................................W167

Villringer, Arno ................................................................................................ W81

Vincent, John ................................................................................................... T171

Vingerhoets, Claudia .................................................................................... M181

Vinogradov, Sophia .................................................................................................... 52

Violett, Coralie ........................................................................................................ W108

Vitaro, Frank .................................................................................................. T238

Viviano, Joseph .............................................................................................168

Vlasova, Roza .................................................................................................... W185

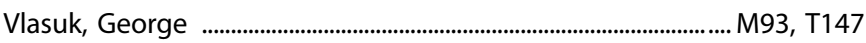

Voineskos, Aristotle .......................................... M168, M191, T92, T225, W35, W167, W168, W170, W178

Vo-Le, Brittany .............................................................................................. W28

Vo-Le, Bylinda ....................................................................................................... W28

Volkmann, Robert ......................................................................................... T226

Volkow, Nora

.50, M67, M249, M275, T253, T285

Voll, Ronald

... W66

Volmar, Claude-Henry ............................................................................... W6, W7

Voloudakis, Georgios .................................................................................. T222

von Deneen, Karen ......................................................................................... M67

Voon, Valerie .................................................................................................... W157

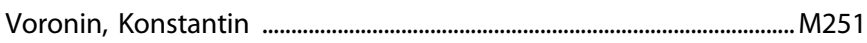

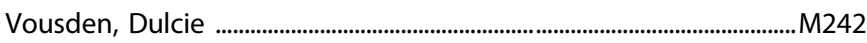

Voytek, Bradley .................................................................................................. T40

Vsevolozhskaya, Olga .................................................................................... W273

Vu, Jimmy ......................................................................................................... W2 W

Vucevic, Danijela ................................................................................................... W212

Vyas, Gopal R. ..............................................................................................M160

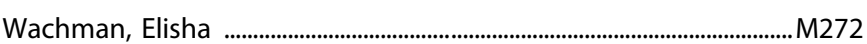

Wada, Masataka ......................................................................................... W162

Wade, Benjamin ...............................................................................................T29

Wadsak, Wolfgang .................................................................................... W127

Wadsworth, Annie ......................................................................................... T173

Wadsworth, Paul ............................................................................................ T173
Waeiss, Robert A. ........................................................................................... T280

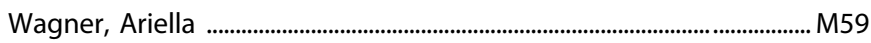

Wagner, Ryan ......................................................................... T183, W11, W287

Wagner, Sarah ............................................................................................. W19

Wahlestedt, Claes …………………………………………........W6, W7, W229

Wainberg, Milton L. .................................................................................... M52

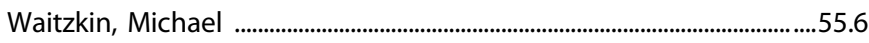

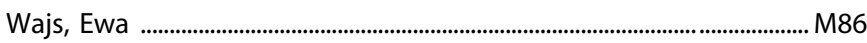

Wakabayashi, Ken ........................................................................................M257

Wake, Hidenori ............................................................................................................. M82

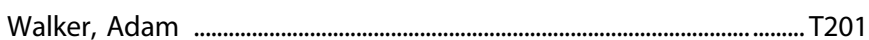

Walker, Brendan ...............................................................................................M260

Walker, Brittni ...........................................................................................................W6

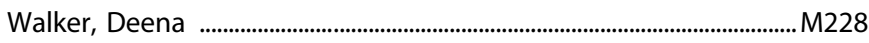

Wall, Melanie .......................................................................................................M164

Walsh, Jessica .................................................................................................. T89

Walss-Bass, Consuelo ..................................................................... W98, W237

Walter, Henrik ......................................................................................................W8

Walter, Sebastian ..................................................................................... W170

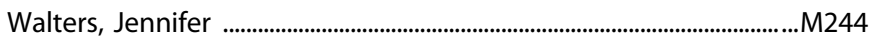

Walther, Sebastian ........................................................................................ W167

Waltz, James ........................................................................................................ W179

Wang, Anna ......................................................................................................189

Wang, Feiran ........................................................................................................... W205

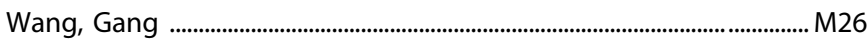

Wang, Gene-Jack ....................................................... M67, M249, M275, T253

Wang, Harold ........................................................................................................ W52

Wang, Jia ................................................................................................................. M67

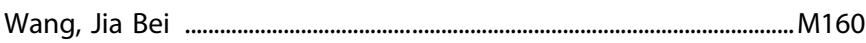

Wang, JiJun ........................................................................................................ W18

Wang, Jingtao .........................................................................................................M160

Wang, Jui-Kai .................................................................................................. T181

Wang, Jun ..................................................................................................... M13

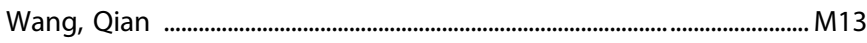

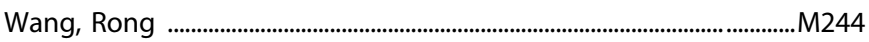

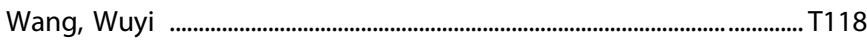

Wang, Yulu ....................................................................................................... W151

Wang, Yun .................................................................................................... W49

Waninger, Shani ...................................................................................................W96

Wanner, Julianne ................................................................................................... T106

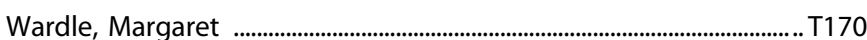

Warren, Brandon ................................................................................................ T55

Wassum, Kate .......................................................................................................... 44

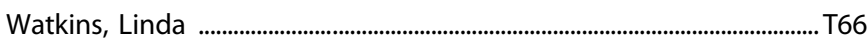

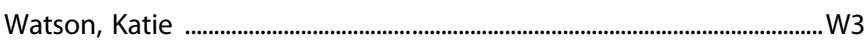

Watson, Philip ................................................................................................W169

Watson, Steve ....................................................................................................193

Watson Lin, Katie ......................................................................................W89

Watts, Jeremy .............................................................................................. W175

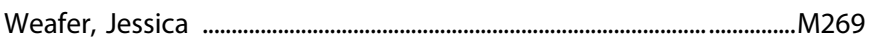

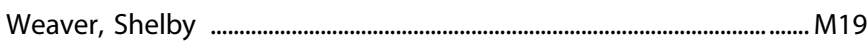

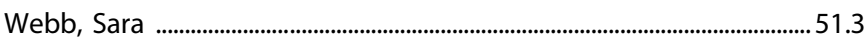

Weber, Wade .................................................................................................................. W91

Weber-Fahr, Wolfgang .................................................................................... W182

Webster, Irena ................................................................................... M143, M144

Weddington, Javier ........................................................................................... W144 
Weedin, Nathan ……………………………………………………..... T138

Weerts, Elise ................................................................................................ T272

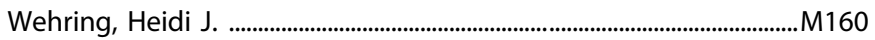

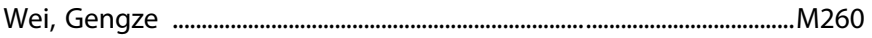

Wei, Heather ...................................................................................................... M88

Wei, Shau-Ming ………………………………………………………….... T166

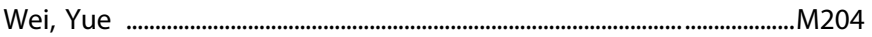

Weigard, Alexander ...................................................................................... W235

Wein, Rachel .......................................................................................165, T209

Weinberger, Daniel ................................................... M175, M176, M199, T224

Weiner, Jeff ................................................................................................... T277

Weiser, Mark ............................................................................................. W56, W180

Weiss, Elizabeth ...................................................................................................T76

Weiss, Hannah ....................................................................................................... M57

Weissman, Myrna ................................................................................ M134, W106

Welch, Amanda ..............................................................................................W69

Welge, Jeffrey ....................................................................................................W91

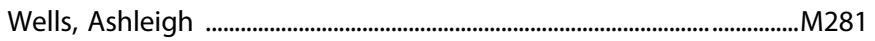

Wells, Lisa A. ...............................................................................................M174

Wels, Joseph .....................................................................................................M142

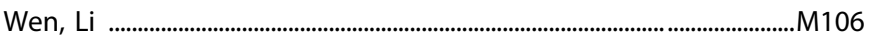

Wengler, Kenneth ......................................................................................... W210

Wennerholm, Michelle ...............................................................................W100

Wenzel, Jennifer ..................................................................................................T5

Wenzel, Julia ......................................................................................................W8

Werneburg, Brian .....................................................................................................W79

West, Elizabeth .................................................................................................M262

Wetherill, Reagan ............................................................................... M266, W263

Wey, Hsiao-Ying ..........................................................................................T269

Wheeler, Alexa-Rae ............................................................. M4, W70, W72, W255

Wheeler, Anne ................................................................................................... W35

White, Amanda ..................................................................................................W29

White, Emma ............................................................................................. W261

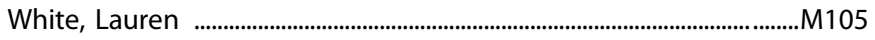

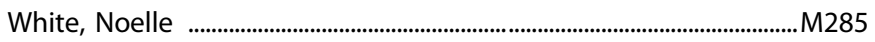

White, Stormi P. ................................................................................................. M47

Whitfield-Gabrieli, Susan .......................................................34.3, M3, W188

Whiting, Kimberly ...........................................................................................M1 16

Whyte, Ellen ..................................................................................M130, W178

Widge, Alik ................................................................................................. 52.1, T74

Wiechmann, Tobias ………………………………………………………….... T22

Wielgosz, Joseph .........................................................................T26, W132

Wiers, Corinde ........................................................................ M249, M275, T253

Wiers, Reinout ........................................................................................................ W13

Wijtenburg, Andrea .......................................................................................189

Wild, Christopher ................................................................................................M238

Wilkerson, Matthew ...................................................................................... W108

Wilkinson, Samuel ........................................................................................ M91

Willard, Alexis ..................................................................................................... W242

Williams, Aislinn ............................................................................................. W28

Williams, Leanne .......................................................7.3, 34.1, T26, T71, W132

Williams, Samuel ........................................................................................ W111

Williams, Sidney ......................................................................................................M250

Wills, Zachary ........................................................................................................... M195

Willuhn, Ingo ............................................................................................................W71

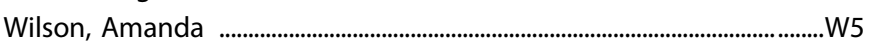

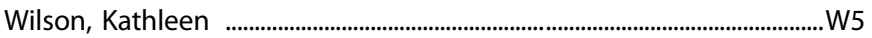

Wilson, Michael ............................................................................................... W207

Wimmer, Mathieu ......................................................................................... T234

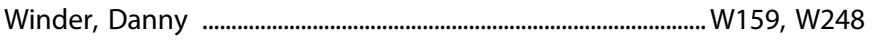

Windisch, Kyle .................................................................................................M256

Windpassinger, Marita ............................................................................W127

Winham, Stacey ............................................................................................ W137

Winkelman, John ............................................................................................10.1

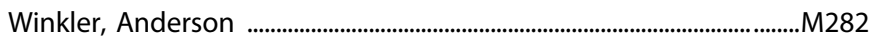

Winnebeck, Emilia ................................................................................................ W84

Winters, Boyer W ....................................................................................... W232

Withers, Dominic J. ...................................................................................M174

Withey, Sarah ....................................................................................... T266, T270

Witt, Shawna .......................................................................................................M207

Wittenberg, Gayle ................................................................................. 45.4, M108

Witwer, Kenneth .............................................................................................. T190

Woelfl, Timo .....................................................................................................M187

Wohleb, Eric ................................................................................................. T141

Wojcik, Joanne ........................................................................................... W167

Wold, Eric .........................................................................................................M238

Wolf, Colten ................................................................................................... T58

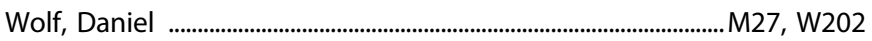

Wolf, Marina .......................................................................................................M225

Wolf, Robert Christian ................................................................................... W165

Wolitzky-Taylor, Kate ..................................................................................... W24

Woller, Sarah A. ...................................................................................................... T176

Womack, Samuel ....................................................................................... T272

Wong, Brian ............................................................................................................124

Wong, Clara .......................................................................................................T47

Wong, Wan-wa ................................................................................................ W158

Wood, Levi .................................................................................................................... T163

Woodruff, Grace ............................................................................................. W100

Woods, James ............................................................................................. W244

Woods, Nick .....................................................................................................M140

Woods, Roger ...........................................................................................................T2

Woodside, Blake .......................................................................................... T120

Woodward, John .............................................................................................. T61

Woodward, Neil ................................................................................................... W16

Woolley, Joshua ............................................................................................... W120

Woolwine, Bobbi ..................................................................................M135, W101

Wooner, Erica ..................................................................................................... M252

Worhunsky \#, Patrick D. .............................................................................M218

Working group, ENIGMA-PGC PTSD neuroimaging ................................T85

Worthen, Shannon .......................................................................................... T139

Wray, Naomi ............................................................................................................. W1 118

Wright, Clinton B. .................................................................................... T176

Wright, Noelle .......................................................................................M4, W72

Wright, William ........................................................................................W231

Wroolie, Tonita .................................................................................................W3

Wu, Baolin .......................................................................................................... T78

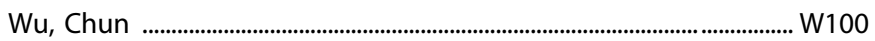

Wu, Nyantsz ......................................................................................................... W152

Wu, Ruyan ................................................................................................................. W216

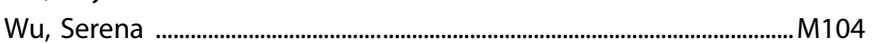

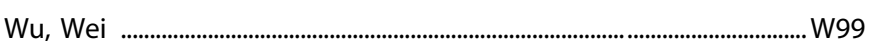

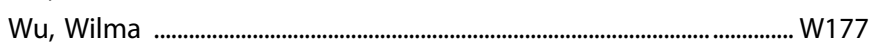


Wu, Yu-Chien

Wulf, Hidegard

Wulff, Rachel

T113

Wurfel, Brent

M108

Wynn, Jonathan

T203, T209

Xi, Zheng-Xiong

T50, W271

Xia, Kai

Xia, Yan W205

Xiang, Zixiu

.T132

Xiao, Lan

T26, W132

Xiao, Yuan W163

Xie, Jason

...M210

$\mathrm{Xu}$, Ashley

M215

$\mathrm{Xu}$, Chengai

M173

$X u$, Haiyan

... 886

$X u$, Junqian

... M46

$\mathrm{Xu}, \mathrm{Ke}$

W233

$\mathrm{Xu}, \mathrm{Li}$

W251

$X u$, Lin

W74

$X u$, Mengxiang

M106

$X u$, Rengyi

...M103

$\mathrm{Xu}$, Ying

M26, W151

Yadav, Vijay . T223

Yakimenko, Olga

T196

Yamaguchi, Hiroshi

W223

Yamajuku, Daisuke

T178

Yamamuro, Kazuhiko

W282

Yamazaki, Mayako

.T206

Yan, Zhen

46.5

Yang, Eddy

... T45

Yang, Shu-Yu

... M76

Yang, Yuan Ru

W270

Yang, Zhongli

M106, T244

Yankey, Jon

M146

Yano, Hideaki

W181

Yao, Emily

Yao, Xiang

T192, W100

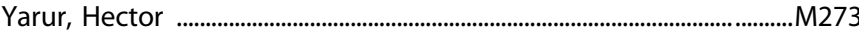

Yaseen, Samina ...................................................................................M200

Yechoor, Vijay

...M162

Yeh, Hung-Wen

W27, W41

Yehl, Jennifer T149

Yehuda, Rachel

39.1, 39.7, M59, T282

Yen, Will

M272

Ying, Noelle

M88

Yingling, Michael

M196

Yip, Sarah

14.3

Yocca, Frank

M72, T230

Yogi, Koudai ....W1

Yohn, Christine

M126, T140

Yohn, Samantha . T132

Yolken, Robert .. M90

Yoo, Hye Bin M216

Yoo, Shinjae
Yoon, Hye-Jean T115

YorkWilliams, Sophie ........................................................................................ W220

Yoshida, Kazunari ......................................................................................... W196

Young, Allan ................................................................................................... W111

Young, Kimberly ............................................................................................. W263

Young, Larry .......................................................................................................... M42

Young, Shannon ..............................................................................................T65

Youngstrom, Eric ................................................................................ T134, T135

Youngstrom, Jennifer ................................................................................. T134

Youssef, Nagy A. ................................................................................................ W138

Yttredahl, Ashley ................................................................................................W97

Yu, Meichen ................................................................................................... W283

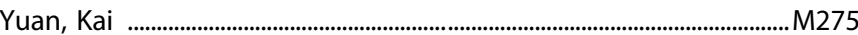

Yuan, Minlan ...................................................................................................... T153

Yuan, Peixiong .................................................................................................. T161

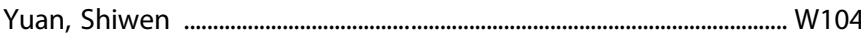

Yuan, Tifei ........................................................................................................ W262

Yuen, Eunice ......................................................................................................... T9

Yuille, Marie ..................................................................................................

Yurgelun-Todd, Deborah ......................................................................... T114

Yusofova, Lyubov .........................................................................................

Yussuf, Zahra ......................................................................................................W62

Yuste, Rafael ..................................................................................................................M198

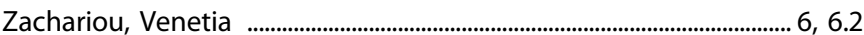

Zachry, Jennifer ................................................................................................ T279

Zack, Sanno .............................................................................................................. T76

Zadeh, Jenny …………………………………………………………………...... T193

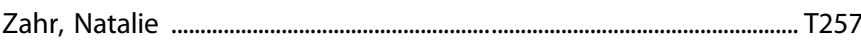

Zai, Clement ........................................................................................................... T171

Zai, Gwyneth .......................................................................................... 47.5 T35

Zaiko, Yevgeniya .........................................................................................T76

Zakzanis, Konstantine ........................................................................... T225

Zamzow, Madeline J. ..................................................................................... W98

Zand Vakili, Amin .........................................................................................115

Zanderigo, Francesca ........................................................................ T116, T153

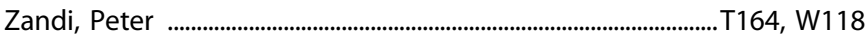

Zanos, Panos ................................................................................... M111, T137

Zarate, Carlos ......................................M141, M232, T144, T161, T164, W133

Zaykin, Dmitri ........................................................................................... W273

Ze, Zengyou ......................................................................................................... M51

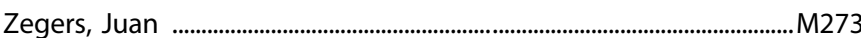

Zekovic, Janko ....................................................................................................... W212

Zelli, Danielle ........................................................................................................ T142

Zeng, Fanxing ............................................................................................................W66

Zeng, Jiaxin .................................................................................................... W163

Zeni, Cristian P. ........................................................................................... T143

Zetterberg, Henrik .........................................................................M173, T158

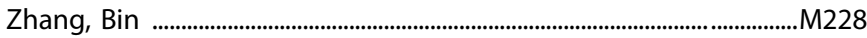

Zhang, Chencheng ................................................................................... W157

Zhang, Eric ......................................................................................................... M265

Zhang, Fan .................................................................................T200, W170

Zhang, Han-Ting ............................................................................................... M26

Zhang, Huaibo ................................................................................................ T271

Zhang, Huailin .......................................................................................................... W234

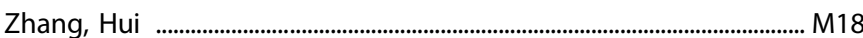


Zhang, Jasmine .. T188

Zhang, Jenny . T242

Zhang, Jiancheng ..W95

Zhang, Jing M213

Zhang, Lan-Yuan ...T53

Zhang, Lianqing M156

Zhang, Lu . T163

Zhang, Rui M249

Zhang, Ruihan W144

Zhang, Sheng $\mathrm{M} 222, \mathrm{~T} 118$

Zhang, Tianhong

Zhang, Tie-Yuan W188

Zhang, Wen

Zhang, Wenchao

Zhang, Wenjing

Zhang, Xu

Zhang, Yan

Zhang, $\mathrm{Yi}$

Zhang, Yu

Zhang, Yun

Zhao, Di

Zhao, Joshua

Zhao, Xuefang

Zhao, Zhongming

Zheng, Ming-Qiang

Zheng, Victor

Zheng, Wang W163 ...W9 M53 M67 ...T76 M86 W262 ...T59 .. W52 W237 M218 W151 W157
Zhornitsky, Simon

M36, M222, T118

Zhou, Jia M238, T173

Zhou, Jing W207

Zhou, Xianxiao M228

Zhou, Yan M256

Zhou, Yang 36.4

Zhu, ManHua

M229

Ziegler, Thomas

W92, W125

Zimmer, Andreas

50.4

Zimmer, Luc

W280

Zipursky, Robert

T194

Zisook, Sidney

Zitkovsky, Emily

M32

Zlebnik, Natalie ...T53

Zonca, Valentina M92
Zong, Wei

W241

Zorick, Todd

M149

Zucker, Robert

W235

M138

Zuiker, Rob W74

Zukin, R. Suzanne ... 16

Zulueta, John ...T65

Zuniga, Edward T249

Zunta-Soares, Giovana

T143

Zuo, Chun .W8

Zweifel, Larry
Zugman, Andre 